\title{
RADIAL DISTRIBUTION OF STARS, GAS, AND DUST IN SINGS GALAXIES. II. DERIVED DUST PROPERTIES
}

\author{
J. C. Muñoz-Mateos ${ }^{1}$, A. Gil de Paz ${ }^{1}$, S. Boissier ${ }^{2}$, J. Zamorano ${ }^{1}$, D. A. Dale ${ }^{3}$, P. G. Pérez-González ${ }^{1}$, \\ J. Gallego ${ }^{1}$, B. F. Madore ${ }^{4}$, G. Bendo ${ }^{5}$, M. D. Thornley ${ }^{6}$, B. T. Draine ${ }^{7}$, A. Boselli² ${ }^{2}$, V. Buat ${ }^{2}$, D. Calzetti ${ }^{8}$, \\ J. MoustaKas ${ }^{9}$, And R. C. Kennicutt, JR. ${ }^{10,11}$ \\ ${ }^{1}$ Departamento de Astrofísica y CC. de la Atmósfera, Universidad Complutense de Madrid, Avda. de la Complutense, s/n, E-28040 Madrid, Spain; \\ jcmunoz@astrax.fis.ucm.es, agpaz@astrax.fis.ucm.es, jaz@astrax.fis.ucm.es, pgperez@astrax.fis.ucm.es, jgm@astrax.fis.ucm.es \\ ${ }^{2}$ Laboratoire d'Astrophysique de Marseille, OAMP, Université Aix-Marseille \& CNRS UMR 6110, 38 rue Frédéric Joliot-Curie, 13388 Marseille cedex 13, France; \\ samuel.boissier@oamp.fr, alessandro.boselli@oamp.fr, veronique.buat@oamp.fr \\ ${ }^{3}$ Department of Physics and Astronomy, University of Wyoming, Laramie, WY, USA; ddale@uwyo.edu \\ ${ }^{4}$ Observatories of the Carnegie Institution of Washington, 813 Santa Barbara Street, Pasadena, CA 91101, USA; barry@ociw.edu \\ ${ }^{5}$ Astrophysics Group, Imperial College, Blackett Laboratory, Prince Consort Road, London SW7 2AZ, UK; g.bendo@imperial.ac.uk \\ ${ }^{6}$ Department of Physics and Astronomy, Bucknell University, Lewisburg, PA 17837, USA; m.thornley@bucknell.edu \\ ${ }^{7}$ Princeton University Observatory, Princeton, NJ 08544-1001, USA; draine@ astro.princeton.edu \\ ${ }^{8}$ Department of Astronomy, University of Massachusetts, Amherst, MA 01003, USA; calzetti@astro.umass.edu \\ ${ }^{9}$ Department of Physics, New York University, 4 Washington Place, New York, NY 10003, USA \\ ${ }^{10}$ Institute of Astronomy, University of Cambridge, Madingley Road, Cambridge CB3 0HA, UK \\ ${ }^{11}$ Steward Observatory, University of Arizona, Tucson, AZ 85721, USA \\ Received 2009 April 15; accepted 2009 July 7; published 2009 August 7
}

\section{ABSTRACT}

We present a detailed analysis of the radial distribution of dust properties in the SINGS sample, performed on a set of ultraviolet (UV), infrared (IR), and H I surface brightness profiles, combined with published molecular gas profiles and metallicity gradients. The internal extinction, derived from the total-IR (TIR)-to-far-UV (FUV) luminosity ratio, decreases with radius, and is larger in Sb-Sbc galaxies. The TIR-to-FUV ratio correlates with the UV spectral slope $\beta$, following a sequence shifted to redder UV colors with respect to that of starbursts. The star formation history (SFH) is identified as the main driver of this departure. Both $L_{\mathrm{TIR}} / L_{\mathrm{FUV}}$ and $\beta$ correlate well with metallicity, especially in moderately face-on galaxies. The relation shifts to redder colors with increased scatter in more edgeon objects. By applying physical dust models to our radial spectral energy distributions, we have derived radial profiles of the total dust mass surface density, the fraction of the total dust mass contributed by polycyclic aromatic hydrocarbons (PAHs), and the intensity of the radiation field heating the grains. The dust profiles are exponential, their radial scale length being constant from $\mathrm{Sb}$ to $\mathrm{Sd}$ galaxies (only $\sim 10 \%$ larger than the stellar scale length). Many S0/a-Sab galaxies have central depressions in their dust radial distributions. The PAH abundance increases with metallicity for $12+\log (\mathrm{O} / \mathrm{H})<9$, and at larger metallicities the trend flattens and even reverses, with the SFH being a plausible underlying driver for this behavior. The dust-to-gas ratio is also well correlated with metallicity and therefore decreases with galactocentric radius. Although most of the total emitted IR power (especially in the outer regions of disks) is contributed by dust grains heated by diffuse starlight with a similar intensity as the local Milky Way radiation field, a small amount of the dust mass $(\sim 1 \%)$ is required to be exposed to very intense starlight in order to reproduce the observed fluxes at $24 \mu \mathrm{m}$, accounting for $\sim 10 \%$ of the total integrated IR power.

Key words: dust, extinction - galaxies: ISM - infrared: galaxies - ultraviolet: galaxies

Online-only material: figure sets, machine-readable tables

\section{INTRODUCTION}

Understanding the spatial distribution of interstellar dust is of particular importance for two reasons: it affects our view of galaxies at different wavelengths, by absorbing ultraviolet (UV) and optical light and re-emitting it in the infrared (IR), and it also constitutes an important element in the chemical evolution of the interstellar medium (ISM). As for the first issue, correcting for dust extinction is usually the main source of uncertainty when deriving properties such as the star formation rate (SFR), age, or metallicity (Calzetti et al. 1994; Buat \& Xu 1996; Calzetti 2001; Pérez-González et al. 2003). This limitation not only applies to integrated data, but also to surface brightness profiles and color gradients. For instance, the so-called inside-out scenario for the formation of galactic disks predicts that the timescale of gas infall and conversion into stars increases with radius, leading to radial variations of the star formation history (SFH), which are in turn observationally translated into color gradients (de Jong
1996; Bell \& de Jong 2000; MacArthur et al. 2004; Taylor et al. 2005). Given that the dust content also changes with radius, it is key to properly quantify that variation with direct tracers of dust extinction; otherwise, derived parameters such as the radial growth rate of disks might be biased (see Muñoz-Mateos et al. 2007 and references therein).

While being an inconvenience when studying star formation, dust itself is also a key ingredient in the chemical enrichment of the ISM. Metals resulting from stellar nucleosynthesis are returned to the ISM, where they condense to form dust grains, some of which are later destroyed and incorporated into new generations of stars. Elements that later condense to form dust grains are injected into the ISM at different rates (see, e.g., Dwek et al. 2009 and references therein). For instance, carbon now locked up into polycyclic aromatic hydrocarbons (PAHs) might have been originally produced in asymptotic giant branch (AGB) stars, whose typical lifetimes are a few Gyr. Other elements now constituting larger grains may have been synthesized in 
more massive stars, which die as supernovae ( $\mathrm{SNe}$ ) in shorter timescales. Thus, the PAH abundance and the dust-to-gas ratio are expected to vary with the age of the stellar populations and correlate with the metal (oxygen) abundance of the gas (see, e.g., Galliano et al. (2008) and references therein). Note, however, that dust formation results from a long chain of poorly understood processes, of which stellar lifetimes and yields are only the first link. Grain growth and destruction in the ISM must also be considered. Indeed, it is thought that only $10 \%$ of interstellar dust is directly formed in stellar sources, with the remaining $90 \%$ being later condensed in the ISM (see Draine 2009 for a recent review on the subject).

Addressing these important issues requires a multiwavelength, multi-object spatially resolved analysis, which is now possible for nearby galaxies thanks to the Spitzer Infrared Nearby Galaxies Survey (SINGS; Kennicutt et al. 2003). The SINGS project has made use of the Spitzer Space Telescope (Werner et al. 2004) to collect IR data, as well as ancillary data from other facilities, for a sample of 75 nearby galaxies, representative of the galaxy population in the local universe. Together with UV images from the Galaxy Evolution Explorer (GALEX; Martin et al. 2005) and H I maps from The H I Nearby Galaxies Survey (THINGS; Walter et al. 2008), this data set provides the scientific community with unprecedented spectral and spatial coverage of the most representative nearby galaxies.

In this paper, we exploit the unique data set resulting from the GALEX-SINGS-THINGS collaboration by obtaining multiwavelength radial profiles for the 57 galaxies in the SINGS sample which are detected and resolved at the longest wavelengths. Our subsample still includes galaxies representative of different morphological types (ellipticals, lenticulars, spirals, and irregulars). The profiles themselves, along with other observational parameters such as asymptotic magnitudes and morphological estimators, can be found in the accompanying paper MuñozMateos et al. (2009, Paper I hereafter). The present paper focuses on the radial distribution of dust properties. Finally, by comparing the profiles of the spirals in the sample with models for the chemical and spectrophotometric evolution of spiral galaxies (Boissier \& Prantzos 1999), we are studying the radial variation of the SFH in these galaxies in a self-consistent frame, where radial changes in the gas infall and chemical enrichment are considered. The attenuation profiles turn out to be essential to properly accommodate the model predictions with the observed profiles. Ignoring extinction not only biases the overall flux level in the UV and optical bands, but also the different radial scale lengths at these wavelengths, which are a direct result of the inside-out assembly of disks. The results of this comparison with theoretical models will be presented in a forthcoming paper and, combined with the present study of the dust properties, should help to better understand how the different components of spiral galaxies get assembled together.

This paper is organized as follows. In Section 2, we briefly outline the main properties of the sample, describe the different data used in this work, and explain how the radial profiles were obtained. Section 3 deals with the radial distribution of internal extinction, and how it relates with other properties such as the UV color, inclination, or metallicity. In Section 4, we compare our profiles with the dust models of Draine \& Li (2007) (DL07 hereafter), and analyze the radial variation of the PAH abundance, the dust mass and luminosity surface densities, the intensity of the heating radiation field and the dust-to-gas ratio. Finally, we summarize our main conclusions in Section 5. In Appendix A, we provide some empirical relationships between the dust properties derived from the model and the observed flux densities at the near- to far-IR bands. In Appendix B, we discuss the possible systematic factors that might affect our results in deriving the parameters of the dust models of DL07.

\section{THE SAMPLE, DATA, AND PROCEDURE}

The SINGS sample (Kennicutt et al. 2003) consists of 75 nearby galaxies which span the range in morphological type, luminosity, and FIR/optical luminosity observed in the local universe. The SINGS galaxies were also selected to cover a reasonably wide range in other additional properties, such as nuclear activity, spiral and bar structure, inclination, surface brightness, and environment. Note, however, that no significant luminous or ultraluminous IR galaxy (that is, with $L_{\mathrm{IR}}>$ $\left.10^{11} L_{\odot}\right)$ is included in the sample. The median distance of the SINGS galaxies is $10 \mathrm{Mpc}$, with all objects being closer than $30 \mathrm{Mpc}$. Since the sample is neither flux- nor volume-limited, its statistical power as a whole is limited. Nevertheless, the wealth of panchromatic data available for these galaxies, together with their proximity, makes it possible to carry out detailed studies on the physics of star formation at kpc scales, including the interplay between star formation and the ISM.

Eighteen SINGS galaxies were not suitable for our purposes and were excluded from the present study.

1. Seven galaxies, DDO 154, DDO 165, Holmberg IX, M81 Dw a, M81 Dw b, NGC 0584, and NGC 4552, are not (or marginally) detected in at least one of the MIPS bands.

2. Nine galaxies, Mrk 33, NGC 1266, NGC 1377, NGC 1482, NGC 2798, NGC 3265, NGC 3773, NGC 5195, and NGC 7552, are unresolved in the MIPS bands. Thus, radial profiles for these galaxies would reflect the shape of the point-spread function (PSF) rather than real structures. In NGC 7552, some spatially extended emission can be discerned, but it is too faint compared to the extremely bright nucleus.

3. The only evident IR source within the optical extent of NGC 1404 at 70 and $160 \mu \mathrm{m}$ is an off-center object at the northeast of the frame. Since it might not be related to NGC 1404, we also excluded this galaxy.

4. The bright center of NGC 3034 saturates the MIPS $24 \mu \mathrm{m}$ detector, thus precluding reliable photometry.

Therefore, the final subsample consists of 57 galaxies, whose main properties are summarized in Table 1 .

\subsection{GALEX Data}

Nearly all SINGS galaxies have been observed in the farUV (FUV; $\lambda_{\text {eff }}=151.6 \mathrm{~nm}$ ) and near-UV (NUV; $\lambda_{\text {eff }}=$ $226.7 \mathrm{~nm}$ ) by GALEX (Martin et al. 2005; see Table 1). The observations are performed simultaneously at both bands thanks to a beam splitter, but some galaxies lack FUV images since the corresponding detector had to be turned off due to intense solar activity or overcurrent events. The delivered images have a final pixel scale of 1 ".5. Although the size of the PSF depends slightly on the position on the detector and the brightness of the source, the typical FWHM is $5^{\prime \prime}-6^{\prime \prime}$. This resolution is similar to that of the MIPS $24 \mu \mathrm{m}$ images, and corresponds to a physical scale of $\sim 300 \mathrm{pc}$ at $10 \mathrm{Mpc}$, the median distance of the SINGS galaxies. The flux calibration is based on white dwarf standard stars. For the pipeline version used here (the same as in Gil de Paz et al. 2007), the estimated zero-point uncertainty is $0.15 \mathrm{mag}$. 


\subsection{Spitzer Data}

The reader is referred to Paper I for a more detailed description of the Spitzer data used here. Mid- and far-IR observations of the SINGS sample were carried out using the Spitzer Space Telescope (Werner et al. 2004). The Infrared Array Camera (IRAC; Fazio et al. 2004) was used to image the SINGS galaxies at $3.6,4.5,5.8$, and $8.0 \mu \mathrm{m}$. We made use of the images provided in the SINGS Fourth Data Delivery, which are based on the Version 13 Basic Calibrated Data produced by the Spitzer Science Center. The delivered images have a pixel scale of 0 . 75 , and the FWHMs of the PSF at each band are $1^{\prime \prime} .7,1^{\prime \prime} .7$, 1 .'9, and 2".0, respectively. These correspond to spatial scales of $80-100 \mathrm{pc}$ at the median distance of the sample. The estimated photometric uncertainty is $\sim 2 \%$ (Reach et al. 2005). However, the photometry needs to be corrected for the diffuse scattering of incoming photons throughout the IRAC array, ${ }^{12}$ and these corrections have an associated uncertainty of $\sim 10 \%$.

The Multi-band Imaging Photometer (MIPS; Rieke et al. 2004) was used in scan-mapping mode to observe the SINGS galaxies at 24,70 , and $160 \mu \mathrm{m}$. The final frames are delivered with pixel scales of $1^{\prime \prime} .5,4$,.5, and 9'.0, respectively, thus being integer multiples of the pixel scale of the IRAC frames while still properly mapping the MIPS PSF. The corresponding FHWMs are $5.7,16^{\prime \prime}$, and $38^{\prime \prime}$ at each band, probing physical scales of $0.28,0.78$, and $1.84 \mathrm{kpc}$ at $10 \mathrm{Mpc}$. The estimated zero-point errors are $4 \%, 5 \%$, and $12 \%$ at 24,70 , and $160 \mu \mathrm{m}$, respectively (Engelbracht et al. 2007; Gordon et al. 2007; Stansberry et al. 2007).

\subsection{H I Data}

The H i Nearby Galaxy Survey (THINGS; Walter et al. 2008) used the Very Large Array (VLA) to map H i $21 \mathrm{~cm}$ line emission from 34 nearby $(D<15 \mathrm{Mpc})$ galaxies, most of which were also targets of SINGS and the GALEX Nearby Galaxies Survey. The observations were done using the B-, C-, and D-array configurations. For details of data reduction and processing, see Walter et al. (2008). Here, we use profiles derived from naturalweighted moment-zero (integrated intensity) maps. These have a typical angular resolution of $11^{\prime \prime}$ and sensitivity to surface densities as low as $4 \times 10^{19} \mathrm{~cm}^{-2}$ once convolved to our $38^{\prime \prime}$ working resolutions (see below). These radial profiles were kindly provided by A. Leroy and F. Walter. Because THINGS includes data from the compact B array configuration, the maps comfortably recover extended structure in our sources. The column densities in the THINGS maps are estimated to be correct to within $\pm 10 \%$.

\subsection{Surface Brightness Profiles}

The reader is referred to Paper I for a more in-depth description of the procedure followed to obtain the surface brightness radial profiles; here we will just briefly describe the most important steps relevant to this paper. In our study of the spatial distribution of the dust properties, we are limited by the resolution of the MIPS $160 \mu \mathrm{m}$ images (FWHM of $38^{\prime \prime}$ ). We convolved the GALEX, IRAC, MIPS, and H I images with different kernels (see Gordon et al. 2008) in order to match the shapes and resolution of their PSFs to the MIPS $160 \mu \mathrm{m}$ PSF. Prior to convolving the images, foreground stars, background galaxies, and artifacts were masked and interpolated over to avoid contamination when degrading the images.

\footnotetext{
12 http://ssc.spitzer.caltech.edu/irac/calib/extcal/
}

The radial profiles were obtained using the $\operatorname{IRAF}^{13}$ task ELLIPSE, measuring the mean intensity along elliptical isophotes with fixed ellipticity and position angle, equal to those of the $\mu_{B}=25 \mathrm{mag} \operatorname{arcsec}^{-2}$ isophote from the RC3 catalog ${ }^{14}$ (de Vaucouleurs et al. 1991). For those objects for which these parameters were not included in the RC3 catalog, we used the major and minor axis diameters and position angles available in NED. The centers of these elliptical isophotes were set at the coordinates shown in Table 1. The semimajor axes of these ellipses were successively incremented by $48^{\prime \prime}$ (a step larger than the PSF FWHM), to a final radius at least 1.5 times the R25 radius (depending on the extension of each particular galaxy). While using radially varying ellipticities and position angles is useful in detailed studies of galactic structure at a specific wavelength, a panchromatic analysis requires using the same set of fixed elliptical isophotes in all bands to measure the different fluxes in the same regions of each galaxy, and for that matter a fixed PA and ellipticity was found to be as appropriate as any other set of values obtained from a given band.

Uncertainties in the surface photometry include the error of the mean intensity within each isophote, computed assuming Poisson statistics, and the uncertainty in the sky level. The latter comes from high spatial frequency errors (Poisson noise, pixelto-pixel variations) and low spatial frequency ones (flat-fielding errors; Gil de Paz \& Madore 2005).

The radial profiles were corrected for Galactic extinction as in Dale et al. (2007), using the color excesses from the maps of Schlegel et al. (1998) and the extinction curve of Li \& Draine (2001), assuming $R_{V}=3.1$. The final profiles are shown in Table 2.

Note that our radial profiles should be taken with caution in very inclined galaxies. First, although the radial step along the major axis is larger than the FWHM of the PSF, this is not the case along the minor axis in edge-on or close to edge-on galaxies. Moreover, the outer regions might be contaminated by emission from the central ones when performing the azimuthal average. Finally, the observed UV and IR radiation in these systems will likely probe different spatial regions within each galaxy, due to the large amount of dust along the line of sight.

\section{RADIAL DISTRIBUTION OF DUST ATTENUATION}

\subsection{Radial Extinction Profiles}

As part of our analysis of the radial distribution of dust properties, we will first determine the radial variation of internal extinction, which is also necessary to recover the intrinsic radial profiles from FUV to NIR. These kinds of studies have been addressed by several authors following different methodologies. Boissier et al. (2004, 2005, 2007) used the radial change in the total-IR (TIR)-to-FUV ratio to derive radial extinction profiles for nearby galaxies. A similar procedure was followed by Popescu et al. (2005) on a pixel-to-pixel basis for M 101. Prescott et al. (2007) obtained extinction profiles in $\mathrm{H} \alpha$ for the SINGS galaxies by comparing the $\mathrm{H} \alpha$ and $24 \mu \mathrm{m}$ fluxes of individual star-forming regions. The number of distant galaxies seen through a spiral disk can also provide an independent estimation of the extinction (e.g., Holwerda et al. 2005 and references therein).

\footnotetext{
${ }^{13}$ IRAF is distributed by the National Optical Astronomy Observatory, which is operated by the Association of Universities for Research in Astronomy, Inc., under cooperative agreement with the National Science Foundation.

${ }^{14}$ Except for NGC 5194, whose original values were highly affected by its companion galaxy, NGC 5195 .
} 
Table 1

Sample

\begin{tabular}{|c|c|c|c|c|c|c|c|c|c|c|c|}
\hline (1) & $\begin{array}{c}\text { R.A.2000 } \\
\text { (h:m:s) } \\
(2)\end{array}$ & $\begin{array}{c}\text { Decl.2000 } \\
\text { (d:m:s) } \\
(3)\end{array}$ & $\begin{array}{c}2 a \\
(\operatorname{arcmin}) \\
(4)\end{array}$ & $\begin{array}{c}2 b \\
(\operatorname{arcmin}) \\
(5)\end{array}$ & $\begin{array}{c}\text { PA } \\
(\mathrm{deg}) \\
(6) \\
\end{array}$ & $\begin{array}{c}E(B-V) \\
(\mathrm{mag}) \\
(7)\end{array}$ & $\begin{array}{c}T \\
\text { Type } \\
(8) \\
\end{array}$ & $\begin{array}{c}\text { Dist } \\
(\mathrm{Mpc}) \\
(9)\end{array}$ & $\begin{array}{c}G A L E X \\
\text { Data } \\
(10)\end{array}$ & $\begin{array}{c}\text { THINGS } \\
\text { Data } \\
(11)\end{array}$ & $\begin{array}{c}\mathrm{CO} \\
\text { Data } \\
(12) \\
\end{array}$ \\
\hline NGC 0024 & 000956.5 & -245747.3 & 5.8 & 1.3 & 46 & 0.020 & 5 & 8.2 & Yes & No & $\ldots$ \\
\hline NGC 0337 & 005950.1 & -073440.7 & 2.9 & 1.8 & 310 & 0.112 & 7 & 25 & Yes & No & $\ldots$ \\
\hline NGC 0628 & 013641.8 & 154700.5 & 10.5 & 9.5 & 25 & 0.070 & 5 & 11 & Yes & Yes & 1 \\
\hline NGC 0855 & 021403.6 & 275237.8 & 2.6 & 1.0 & 60 & 0.072 & -5 & 9.7 & Yes & No & $\ldots$ \\
\hline NGC 0925 & 022716.9 & 333445.0 & 10.5 & 5.9 & 282 & 0.076 & 7 & 9.3 & Yes & Yes & 2 \\
\hline NGC 1097 & 024619.1 & -301629.7 & 9.3 & 6.3 & 310 & 0.027 & 3 & 15 & Yes & No & 3 \\
\hline NGC 1291 & 031718.6 & -410629.1 & 9.8 & 8.1 & 345 & 0.013 & 0 & 9.7 & Yes & No & $\ldots$ \\
\hline NGC 1316 & 032241.7 & -371229.6 & 12.0 & 8.5 & 50 & 0.021 & -2 & 19 & Yes & No & $\ldots$ \\
\hline NGC 1512 & 040354.3 & -432055.9 & 8.9 & 5.6 & 90 & 0.011 & 1 & 10 & Yes & No & $\ldots$ \\
\hline NGC 1566 & 042000.4 & -545616.1 & 8.3 & 6.6 & 60 & 0.009 & 4 & 17 & Yes & No & 4 \\
\hline NGC 1705 & 045413.5 & -532139.8 & 1.9 & 1.4 & 50 & 0.008 & 11 & 5.1 & Yes & No & $\ldots$ \\
\hline NGC 2403 & 073651.4 & 653609.2 & 21.9 & 12.3 & 307 & 0.040 & 6 & 3.2 & Yes & Yes & 3 \\
\hline Holmberg II & 081905.0 & 704312.1 & 7.9 & 6.3 & 15 & 0.032 & 10 & 3.4 & Yes & Yes & $\ldots$ \\
\hline DDO 053 & 083407.2 & 661054.0 & 1.5 & 1.3 & 300 & 0.037 & 10 & 3.6 & Yes & Yes & $\ldots$ \\
\hline NGC 2841 & 092202.6 & 505835.5 & 8.1 & 3.5 & 327 & 0.016 & 3 & 14 & Yes & Yes & 5 \\
\hline NGC 2915 & 092611.5 & -763734.8 & 1.9 & 1.0 & 309 & 0.275 & 90 & 3.8 & Yes & No & $\ldots$ \\
\hline Holmberg I & 094032.3 & 711056.0 & 3.6 & 3.0 & 360 & 0.048 & 10 & 3.8 & Yes & Yes & $\ldots$ \\
\hline NGC 2976 & 094715.5 & 675459.0 & 5.9 & 2.7 & 323 & 0.069 & 5 & 3.6 & Yes & Yes & $\ldots$ \\
\hline NGC 3049 & 095449.7 & 091617.9 & 2.2 & 1.4 & 25 & 0.038 & 2 & 22 & NUV & No & $\ldots$ \\
\hline NGC 3031 & 095533.2 & 690355.1 & 26.9 & 14.1 & 337 & 0.080 & 2 & 3.6 & Yes & Yes & 2 \\
\hline NGC 3190 & 101805.6 & 214955.0 & 4.4 & 1.5 & 305 & 0.025 & 1 & 17 & Yes & No & $\ldots$ \\
\hline NGC 3184 & 101817.0 & 412528.0 & 7.4 & 6.9 & 135 & 0.017 & 6 & 8.6 & Yes & No & $\ldots$ \\
\hline NGC 3198 & 101954.9 & 453259.0 & 8.5 & 3.3 & 35 & 0.012 & 5 & 17 & Yes & Yes & $\ldots$ \\
\hline IC 2574 & 102823.5 & 682443.7 & 13.2 & 5.4 & 50 & 0.036 & 9 & 4.0 & Yes & Yes & $\ldots$ \\
\hline NGC 3351 & 104357.7 & 114213.0 & 7.4 & 5.0 & 13 & 0.028 & 3 & 12 & Yes & Yes & $\ldots$ \\
\hline NGC 3521 & 110548.6 & -000209.1 & 11.0 & 5.1 & 343 & 0.058 & 4 & 9.0 & Yes & Yes & 1 \\
\hline NGC 3621 & 111816.5 & -324850.6 & 12.3 & 7.1 & 339 & 0.080 & 7 & 8.3 & Yes & Yes & $\ldots$ \\
\hline NGC 3627 & 112015.0 & 125929.6 & 9.1 & 4.2 & 353 & 0.032 & 3 & 9.1 & Yes & Yes & 1 \\
\hline NGC 3938 & 115249.4 & 440714.6 & 5.4 & 4.9 & 15 & 0.021 & 5 & 12 & NUV & No & $\ldots$ \\
\hline NGC 4125 & 120806.0 & 651026.9 & 5.8 & 3.2 & 275 & 0.019 & -5 & 21 & NUV & No & $\ldots$ \\
\hline NGC 4236 & 121642.1 & 692745.3 & 21.9 & 7.2 & 342 & 0.015 & 8 & 4.5 & Yes & No & $\ldots$ \\
\hline NGC 4254 & 121849.6 & 142459.4 & 5.4 & 4.7 & 35 & 0.039 & 5 & 17 & NUV & No & $\ldots$ \\
\hline NGC 4321 & 122254.9 & 154920.6 & 7.4 & 6.3 & 30 & 0.026 & 4 & 18 & NUV & No & $\ldots$ \\
\hline NGC 4450 & 122829.6 & 170505.8 & 5.2 & 3.9 & 355 & 0.028 & 2 & 17 & NUV & No & $\ldots$ \\
\hline NGC 4536 & 123427.1 & 021116.4 & 7.6 & 3.2 & 310 & 0.018 & 4 & 15 & Yes & No & $\ldots$ \\
\hline NGC 4559 & 123557.7 & 275735.1 & 10.7 & 4.4 & 330 & 0.018 & 6 & 17 & Yes & No & $\ldots$ \\
\hline NGC 4569 & 123649.8 & 130946.3 & 9.5 & 4.4 & 23 & 0.046 & 2 & 17 & Yes & No & 1,6 \\
\hline NGC 4579 & 123743.6 & 114905.1 & 5.9 & 4.7 & 275 & 0.041 & 3 & 17 & Yes & No & $\ldots$ \\
\hline NGC 4594 & 123959.4 & -113723.0 & 8.7 & 3.5 & 90 & 0.051 & 1 & 9.1 & Yes & No & $\ldots$ \\
\hline NGC 4625 & 124152.7 & 411625.4 & 2.2 & 1.9 & 330 & 0.018 & 9 & 9.5 & Yes & No & $\ldots$ \\
\hline NGC 4631 & 124208.0 & 323229.4 & 15.5 & 2.7 & 86 & 0.017 & 7 & 9.0 & Yes & No & $\ldots$ \\
\hline NGC 4725 & 125026.6 & 253002.7 & 10.7 & 7.6 & 35 & 0.012 & 2 & 17 & Yes & No & $\ldots$ \\
\hline NGC 4736 & 125053.1 & 410713.6 & 11.2 & 9.1 & 285 & 0.018 & 2 & 5.2 & Yes & Yes & 1 \\
\hline NGC 4826 & 125643.8 & 214051.9 & 10.0 & 5.4 & 295 & 0.041 & 2 & 17 & Yes & Yes & 1 \\
\hline NGC 5033 & 131327.5 & 363538.0 & 10.7 & 5.0 & 170 & 0.011 & 5 & 13 & Yes & No & $\ldots$ \\
\hline NGC 5055 & 131549.3 & 420145.4 & 12.6 & 7.2 & 285 & 0.018 & 4 & 8.2 & Yes & Yes & 1 \\
\hline NGC 5194**** & 132952.7 & 471142.6 & 11.2 & 9.0 & 360 & 0.035 & 4 & 8.4 & Yes & Yes & 7 \\
\hline TOL 89 & 140121.6 & -330349.6 & 2.8 & 1.7 & 352 & 0.066 & 8.1 & 16 & Yes & No & $\ldots$ \\
\hline NGC 5408 & 140320.9 & -412240.0 & 1.6 & 0.8 & 12 & 0.069 & 9.7 & 4.5 & No & No & $\ldots$ \\
\hline NGC 5474 & 140501.6 & 533944.0 & 4.8 & 4.3 & 360 & 0.011 & 6 & 6.8 & Yes & No & $\ldots$ \\
\hline NGC 5713 & 144011.5 & -001721.2 & 2.8 & 2.5 & 10 & 0.039 & 4 & 27 & Yes & No & $\ldots$ \\
\hline NGC 5866 & 150629.6 & 554547.9 & 4.7 & 1.9 & 308 & 0.013 & -1 & 15 & Yes & No & $\ldots$ \\
\hline IC 4710 & 182838.0 & -665856.0 & 3.6 & 2.8 & 5 & 0.089 & 9 & 8.5 & Yes & No & $\ldots$ \\
\hline NGC 6822 & 194456.6 & -144721.4 & 15.5 & 13.5 & 7 & 0.236 & 10 & 0.60 & Yes & No & $\cdots$ \\
\hline NGC 6946 & 203452.3 & 600914.2 & 11.5 & 9.8 & 75 & 0.342 & 6 & 5.5 & Yes & No & $\ldots$ \\
\hline NGC 7331 & 223704.1 & 342456.3 & 10.5 & 3.7 & 351 & 0.091 & 3 & 15 & Yes & Yes & 5 \\
\hline NGC 7793 & 235749.8 & -323527.7 & 9.3 & 6.3 & 278 & 0.019 & 7 & 2.0 & Yes & Yes & $\ldots$ \\
\hline
\end{tabular}

Notes. Sample. (1) Galaxy name. (2) R.A. (J2000) of the galaxy center. (3) Decl. (J2000) of the galaxy center. (4, 5) Apparent major and minor isophotal diameters at $\mu_{B}=25 \mathrm{mag} \operatorname{arcsec}^{-2}$ from the RC3 catalog. (6) Position angle from RC3.***The PA and axis ratio for NGC 5194 differ from those in the RC3, which are affected by the presence of NGC 5195. (7) Galactic color excess from Schlegel et al. (1998). (8) Morphological type $T$ as given in the RC3 catalog. (9) Distance to the galaxy, rounded to the nearest Mpc when larger than $10 \mathrm{Mpc}$, taken from Gil de Paz et al. (2007) and Kennicutt et al. (2003). (10) Available GALEX images. (11) Available H I maps from THINGS. (12) References for the CO data. (1) Regan et al. 2001; (2) Sage 1993; (3) Young et al. 1995; (4) Bajaja et al. 1995; (5) Young \& Scoville 1982; (6) Kenney \& Young 1988; (7) Paglione et al. 2001. 
Table 2

Degraded GALEX, IRAC, and MIPS Profiles

\begin{tabular}{|c|c|c|c|c|c|c|c|c|c|c|}
\hline $\begin{array}{c}r \\
(\operatorname{arcsec}) \\
(1) \\
\end{array}$ & $\begin{array}{c}r \\
(\mathrm{kpc}) \\
(2)\end{array}$ & $\begin{array}{c}\mu_{\mathrm{FUV}} \\
(\mathrm{mag} / \square) \\
(3)\end{array}$ & $\begin{array}{c}\mu_{\mathrm{NUV}} \\
(\mathrm{mag} / \square) \\
(4)\end{array}$ & $\begin{array}{c}\mu_{3.6 \mu \mathrm{m}} \\
(\mathrm{mag} / \square) \\
(5)\end{array}$ & $\begin{array}{c}\mu_{4.5 \mu \mathrm{m}} \\
(\mathrm{mag} / \square) \\
(6)\end{array}$ & $\begin{array}{c}\mu_{5.8 \mu \mathrm{m}} \\
(\mathrm{mag} / \square) \\
(7)\end{array}$ & $\begin{array}{c}\mu_{3.6 \mu \mathrm{m}} \\
(\mathrm{mag} / \square) \\
(8)\end{array}$ & $\begin{array}{c}\mu_{24 \mu \mathrm{m}} \\
(\mathrm{mag} / \square) \\
(9)\end{array}$ & $\begin{array}{c}\mu 70 \mu \mathrm{m} \\
(\mathrm{mag} / \square) \\
(10)\end{array}$ & $\begin{array}{c}\mu_{160 \mu \mathrm{m}} \\
(\mathrm{mag} / \square) \\
(11)\end{array}$ \\
\hline \multicolumn{11}{|l|}{ NGC 7331} \\
\hline 0 & 0.0 & $24.99 \pm 0.02$ & $23.74 \pm 0.01$ & $18.41 \pm 0.01$ & $18.88 \pm 0.01$ & $18.48 \pm 0.01$ & $17.81 \pm 0.01$ & $17.82 \pm 0.01$ & $15.08 \pm 0.01$ & $14.11 \pm 0.01$ \\
\hline 48 & 3.5 & $25.08 \pm 0.01$ & $24.01 \pm 0.00$ & $18.99 \pm 0.01$ & $19.45 \pm 0.01$ & $18.90 \pm 0.01$ & $18.12 \pm 0.01$ & $18.04 \pm 0.01$ & $15.16 \pm 0.01$ & $14.15 \pm 0.01$ \\
\hline 96 & 6.9 & $25.31 \pm 0.01$ & $24.53 \pm 0.01$ & $20.03 \pm 0.01$ & $20.47 \pm 0.01$ & $19.74 \pm 0.01$ & $18.86 \pm 0.01$ & $18.83 \pm 0.01$ & $15.71 \pm 0.01$ & $14.82 \pm 0.01$ \\
\hline 144 & 10.4 & $25.62 \pm 0.01$ & $24.97 \pm 0.01$ & $20.78 \pm 0.01$ & $21.22 \pm 0.01$ & $20.52 \pm 0.02$ & $19.65 \pm 0.01$ & $19.78 \pm 0.01$ & $16.56 \pm 0.01$ & $15.60 \pm 0.01$ \\
\hline 192 & 13.8 & $26.07 \pm 0.01$ & $25.47 \pm 0.01$ & $21.39 \pm 0.01$ & $21.84 \pm 0.02$ & $21.20 \pm 0.04$ & $20.36 \pm 0.02$ & $20.59 \pm 0.02$ & $17.39 \pm 0.03$ & $16.28 \pm 0.01$ \\
\hline 240 & 17.3 & $26.52 \pm 0.01$ & $25.99 \pm 0.02$ & $22.06 \pm 0.02$ & $22.50 \pm 0.03$ & $21.88 \pm 0.07$ & $21.07 \pm 0.04$ & $21.34 \pm 0.04$ & $18.10 \pm 0.05$ & $17.03 \pm 0.02$ \\
\hline 288 & 20.7 & $27.30 \pm 0.01$ & $26.74 \pm 0.04$ & $22.77 \pm 0.04$ & $23.20 \pm 0.06$ & $22.66 \pm 0.14$ & $21.93 \pm 0.09$ & $22.28 \pm 0.10$ & $18.79 \pm 0.09$ & $17.82 \pm 0.04$ \\
\hline 336 & 24.2 & $28.14 \pm 0.03$ & $27.51 \pm 0.08$ & $23.32 \pm 0.07$ & $23.75 \pm 0.10$ & $23.33 \pm 0.26$ & $22.68 \pm 0.17$ & $23.11 \pm 0.22$ & $19.44 \pm 0.16$ & $18.43 \pm 0.07$ \\
\hline 384 & 27.7 & $28.88 \pm 0.05$ & $28.15 \pm 0.15$ & $23.69 \pm 0.09$ & $24.12 \pm 0.14$ & $23.79 \pm 0.39$ & $23.18 \pm 0.28$ & $23.69 \pm 0.37$ & $19.81 \pm 0.22$ & $18.95 \pm 0.22$ \\
\hline 432 & 31.1 & $29.43 \pm 0.07$ & $28.58 \pm 0.22$ & $23.98 \pm 0.12$ & $24.42 \pm 0.18$ & $24.15 \pm 0.54$ & $23.53 \pm 0.38$ & $23.96 \pm 0.47$ & $20.06 \pm 0.28$ & $19.38 \pm 0.17$ \\
\hline
\end{tabular}

Notes. Surface brightness profiles measured on the GALEX, IRAC, and MIPS images, after matching their PSFs to that of the $160 \mu \mathrm{m}$ band. Only the profiles for NGC 7331 are shown as an example in the printed version of the journal. (1) Radius along the semimajor axis in arcseconds. (2) Radius along the semimajor axis in kpc. (3)-(11) Surface brightness at different wavelengths. All values are in AB mag $\operatorname{arcsec}^{-2}$. The uncertainties include photometric and background errors, but not zero-point uncertainties, which are added in quadrature when needed.

(This table is available in its entirety in a machine-readable form in the online journal. A portion is shown here for guidance regarding its form and content.)

Here we follow the first method to infer the radial distribution of dust attenuation for the SINGS galaxies. Several studies (Buat \& Xu 1996; Meurer et al. 1999; Gordon et al. 2000; Witt \& Gordon 2000; Buat et al. 2005) have shown that the TIR-to-UV ratio is a robust tracer of the internal extinction in star-forming galaxies, in the sense that it depends weakly on details such as the relative geometry of stars and dust, the shape of the extinction curve, or the SFH. Regarding this latter issue, Buat et al. (2005) only found significant deviations in systems with very quiescent SFH at present-day (that is, having decayed with exponential timescales $\leqslant 2 \mathrm{Gyr}$ ). In these galaxies, the general starlight radiation field would become an even more important source of dust heating than it already is in normal star-forming galaxies. This precludes computing the internal extinction in ellipticals, lenticulars, and also the bulges of spirals with the same calibration. Using synthetic spectral energy distributions (SEDs) of galaxies with different SFHs and attenuations, Cortese et al. (2008) further investigated the dependence of the TIR-to-FUV ratio on the mean age of the stellar populations, and confirmed that quiescent systems exhibit larger TIR-to-FUV ratios than more actively star-forming ones with the same extinction. To account for the extra dust heating contributed by older stars, they provide an SFH-dependent calibration to estimate the UV attenuation. Note that, as pointed out by these authors, this recipe should not be blindly applied to ellipticals, since their FUV emission does not seem to be linked with recent star formation activity. We include these galaxies here just for completeness, but any further interpretation of their observed TIR-to-FUV ratio in terms of attenuation should be done with caution.

Using the low (48" step) resolution profiles at 8, 24, 70, and $160 \mu \mathrm{m}$, we built TIR $(3-1100 \mu \mathrm{m})$ profiles using the weighted sum proposed by DL07, after having subtracted the stellar emission at 8 and $24 \mu \mathrm{m}$ (see Section 4.2). This estimator of the TIR luminosity constitutes a slight improvement over that of Dale \& Helou (2002) using the MIPS bands alone, to the degree that including the $8 \mu \mathrm{m}$ flux seems to reduce the scatter associated with differences in PAH abundances. ${ }^{15}$

\footnotetext{
15 We have checked that the particular choice of this recipe is not critical, since both estimators yield almost equal TIR luminosities (on average, the TIR values derived from the DL07 calibration are 5\% larger, with an rms of 5\%).
}

We then measured low-resolution profiles on the convolved GALEX FUV and NUV images and estimated $A_{\mathrm{FUV}}$ and $A_{\mathrm{NUV}}$ from the TIR-to-FUV and TIR-to-NUV ratios, respectively. The attenuation at both wavelengths was computed using both the fits of Buat et al. (2005) and those of Cortese et al. (2008). Note that at small subgalactic scales, radiative transfer could limit the usefulness of the TIR-to-UV ratio as an extinction tracer, in the sense that UV photons emerging from a given region could heat dust in another region. Considering that our radial sampling is relatively coarse and that we average our data within elliptical annuli, this effect-if present—-should be small (except maybe in extreme objects with very different UV and IR distributions, which are not common in the SINGS sample).

The extinction profiles we derive are shown in Table 3. In Figure 1, we show all the FUV extinction profiles for the galaxies in the sample, normalized to the optical radius R25. A general trend of $A_{\mathrm{FUV}}$ decreasing with radius is clearly seen. The NUV profiles are not shown, since they exhibit similar behavior as the FUV ones.

The extinction in the top panels has been computed with the calibration of Buat et al. (2005), whereas in the bottom ones we have used the SFH-dependent recipe of Cortese et al. (2008). While the equation relating $L_{\mathrm{TIR}} / L_{\mathrm{FUV}}$ and $A_{\mathrm{FUV}}$ is unique in the case of Buat et al. (2005), Cortese et al. (2008) parameterized the coefficients of such a conversion as a function of observable colors that depend on the overall $\mathrm{SFH}$. We followed the prescriptions given by these authors and determined the particular conversion between $L_{\mathrm{TIR}} / L_{\mathrm{FUV}}$ and $A_{\mathrm{FUV}}$ for each annulus depending on its observed (FUV-H) color. The latter was estimated assuming that $(H-3.6 \mu \mathrm{m}) \sim$ -1.03 which, according to the stellar populations models of Bruzual \& Charlot (2003), is the typical color exhibited by starforming galaxies, and it is quite independent of their SFH.

In S0/a-Sab galaxies and the bulges of later-type galaxies, the attenuation derived from the age-dependent calibration is $\sim 0.5$

The DL07 recipe is a proxy for the actual TIR luminosity derived from the DL07 models, designed to be valid over a wide range of starlight intensities and PAH abundances. In Section 4, we will derive $L_{\mathrm{TIR}}$ from the model fitting, along with the remaining model parameters. On average, for the particular range of starlight intensities and PAH abundances in our sample, the TIR luminosities yielded by the estimator are only $6 \%$ larger than those from the model, with an rms of $3 \%$. 
Table 3

Extinction Profiles

\begin{tabular}{|c|c|c|c|c|c|c|c|}
\hline \multirow[b]{2}{*}{$\begin{array}{c}r \\
(\operatorname{arcsec}) \\
(1)\end{array}$} & \multirow[b]{2}{*}{$\begin{array}{c}r \\
(\mathrm{kpc}) \\
(2) \\
\end{array}$} & \multirow[b]{2}{*}{$\begin{array}{c}\log \left(L_{\mathrm{TIR}} / L_{\mathrm{FUV}}\right) \\
(3) \\
\end{array}$} & \multirow[b]{2}{*}{$\begin{array}{c}\log \left(L_{\mathrm{TIR}} / L_{\mathrm{NUV}}\right) \\
(4) \\
\end{array}$} & \multicolumn{2}{|c|}{ B05 } & \multicolumn{2}{|c|}{$\mathrm{C} 08$} \\
\hline & & & & $\begin{array}{c}A_{\mathrm{FUV}} \\
(\mathrm{mag}) \\
(5) \\
\end{array}$ & $\begin{array}{c}A_{\mathrm{NUV}} \\
(\mathrm{mag}) \\
(6) \\
\end{array}$ & $\begin{array}{c}A_{\mathrm{FUV}} \\
(\mathrm{mag}) \\
(7) \\
\end{array}$ & $\begin{array}{c}A_{\mathrm{NUV}} \\
(\mathrm{mag}) \\
(8) \\
\end{array}$ \\
\hline \multicolumn{8}{|l|}{ NGC 7331} \\
\hline 0 & 0.0 & $1.76 \pm 0.07$ & $1.44 \pm 0.07$ & $3.52_{-0.11}^{+0.11}$ & $2.35_{-0.10}^{+0.10}$ & $2.45_{-0.10}^{+0.10}$ & $1.95_{-0.10}^{+0.10}$ \\
\hline 48 & 3.5 & $1.76 \pm 0.07$ & $1.50 \pm 0.07$ & $3.50_{-0.11}^{+0.11}$ & $2.48_{-0.10}^{+0.10}$ & $2.71_{-0.11}^{+0.11}$ & $2.21_{-0.11}^{+0.11}$ \\
\hline 96 & 6.9 & $1.59 \pm 0.07$ & $1.45 \pm 0.07$ & $3.16_{-0.10}^{+0.10}$ & $2.38_{-0.10}^{+0.10}$ & $2.69_{-0.11}^{+0.11}$ & $2.26_{-0.12}^{+0.12}$ \\
\hline 144 & 10.4 & $1.39 \pm 0.07$ & $1.30 \pm 0.07$ & $2.75_{-0.10}^{+0.10}$ & $2.09_{-0.09}^{+0.09}$ & $2.41_{-0.10}^{+0.10}$ & $2.01_{-0.10}^{+0.10}$ \\
\hline 192 & 13.8 & $1.27 \pm 0.07$ & $1.21 \pm 0.07$ & $2.51_{-0.09}^{+0.09}$ & $1.91_{-0.09}^{+0.09}$ & $2.21_{-0.10}^{+0.10}$ & $1.83_{-0.10}^{+0.10}$ \\
\hline 240 & 17.3 & $1.16 \pm 0.07$ & $1.12 \pm 0.07$ & $2.30_{-0.09}^{+0.09}$ & $1.75_{-0.09}^{+0.09}$ & $2.04_{-0.09}^{+0.09}$ & $1.69_{-0.09}^{+0.09}$ \\
\hline 288 & 20.7 & $1.16 \pm 0.07$ & $1.11 \pm 0.07$ & $2.30_{-0.09}^{+0.09}$ & $1.74_{-0.09}^{+0.09}$ & $2.03_{-0.10}^{+0.10}$ & $1.66_{-0.10}^{+0.10}$ \\
\hline 336 & 24.2 & $1.23 \pm 0.07$ & $1.15 \pm 0.08$ & $2.44_{-0.10}^{+0.10}$ & $1.82_{-0.11}^{+0.11}$ & $2.11_{-0.10}^{+0.10}$ & $1.70_{-0.11}^{+0.11}$ \\
\hline 384 & 27.7 & $1.34 \pm 0.08$ & $1.22 \pm 0.10$ & $2.65_{-0.12}^{+0.12}$ & $1.94_{-0.14}^{+0.14}$ & $2.23_{-0.12}^{+0.12}$ & $1.79_{-0.14}^{+0.14}$ \\
\hline 432 & 31.1 & $1.43 \pm 0.09$ & $1.26 \pm 0.12$ & $2.82_{-0.14}^{+0.14}$ & $2.01_{-0.18}^{+0.18}$ & $2.32_{-0.14}^{+0.14}$ & $1.83_{-0.18}^{+0.18}$ \\
\hline
\end{tabular}

Notes. Radial extinction profiles. (1) Radius along the semimajor axis in arcsec. (2) Radius along the semimajor axis in kpc. (3) TIR-to-FUV ratio. (4) TIR-to-NUV ratio. $(5,6)$ Extinction at FUV and NUV computed with the SFH-independent fits of Buat et al. (2005). (7, 8) Extinction at FUV and NUV computed with the SFH-dependent prescriptions of Cortese et al. (2008).

(This table is available in its entirety in a machine-readable form in the online journal. A portion is shown here for guidance regarding its form and content.)

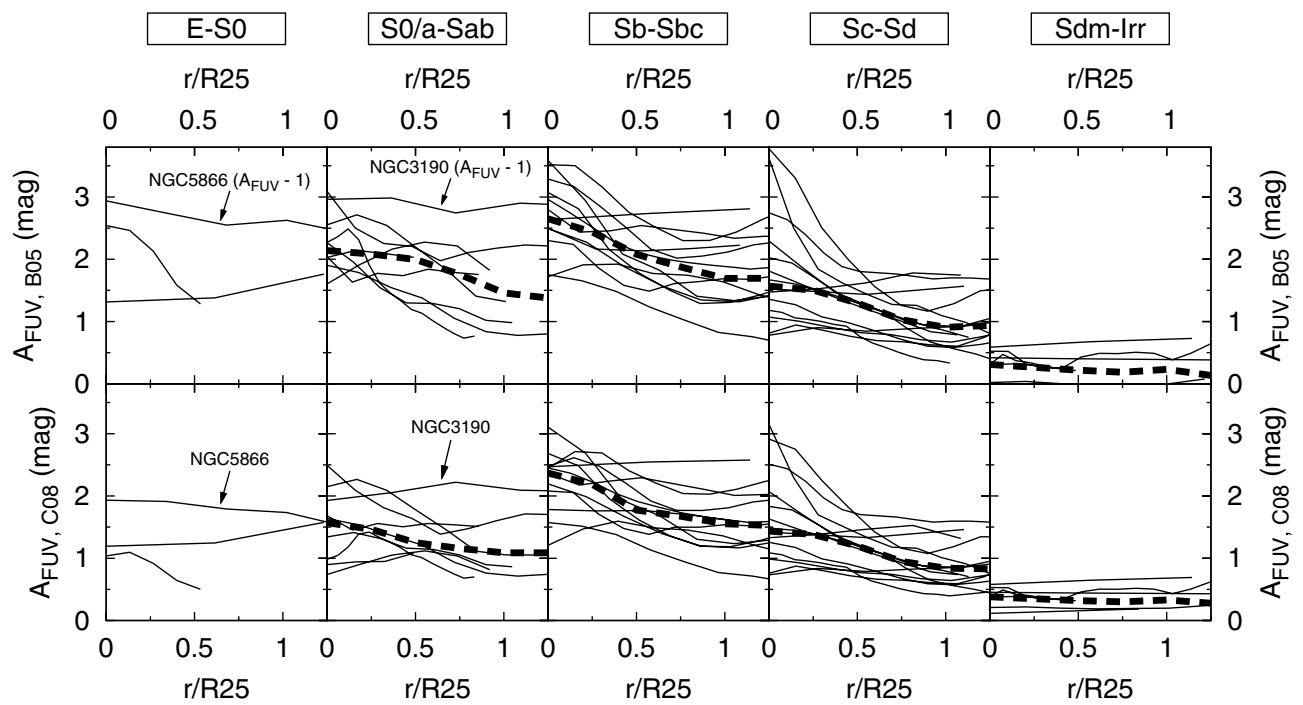

Figure 1. (a) Radial extinction profiles for the SINGS galaxies, computed from the TIR-to-FUV ratio. The horizontal axis is normalized to the optical size of each galaxy. The dashed curves show the median profiles in each bin of morphological types, when enough galaxies are available. Top row: extinction computed using the fit of Buat et al. (2005), which is independent of the SFH. Bottom row: extinction computed from the SFH-dependent calibration of Cortese et al. (2008). The conversion law is explicitly derived for each annulus depending on its observed (FUV-3.6 $\mu \mathrm{m}$ ) color, in order to take into account the dust heating contributed by old stars. For clarity, the profiles of the edge-on galaxies NGC 5866 and NGC 3190 have been offsetted by -1 mag in the top panels (but not in the bottom ones).

mag lower than the one yielded by the age-independent recipe. The difference is negligible in the disk-dominated regions. Given that the SINGS galaxies span a wide range in SFHs, both among them and within different regions of the same object, the results discussed in the rest of the paper refer to the extinction derived following the method of Cortese et al. (2008), except when mentioned otherwise.

The overall level of extinction varies along the Hubble sequence, reaching a maximum in $\mathrm{Sb}-\mathrm{Sbc}$ galaxies. On average, in these spirals the attenuation in the FUV ranges from $\sim 2.5 \mathrm{mag}$ in the central regions to $\sim 1.5 \mathrm{mag}$ in the outer ones, although with large scatter. The extinction is $\sim 1 \mathrm{mag}$ lower in earlier spirals, and it goes below $0.5 \mathrm{mag}$ in Sdm spirals and irregulars.
Note again that the attenuation in ellipticals and lenticulars is highly uncertain, since even the age-dependent recipes of Cortese et al. (2008) might fail in these galaxies.

In order to quantify the attenuation radial gradients, we performed a linear fit to the $A_{\mathrm{FUV}}(r)$ profiles, without including the bulges of spirals for the reasons mentioned above. This exclusion was done visually: bulges produce a steep central rise of the $3.6 \mu \mathrm{m}$ luminosity above the exponential disk, and the FUV emission is significantly reduced in the central regions. This yields a sharp change in the (FUV-3.6 $\mu \mathrm{m}$ ) that, together with a visual inspection of the image, can be used to roughly delimit the bulge- and disk-dominated regions of the profiles (see also Paper I). The results are shown in Figure 2, where the 


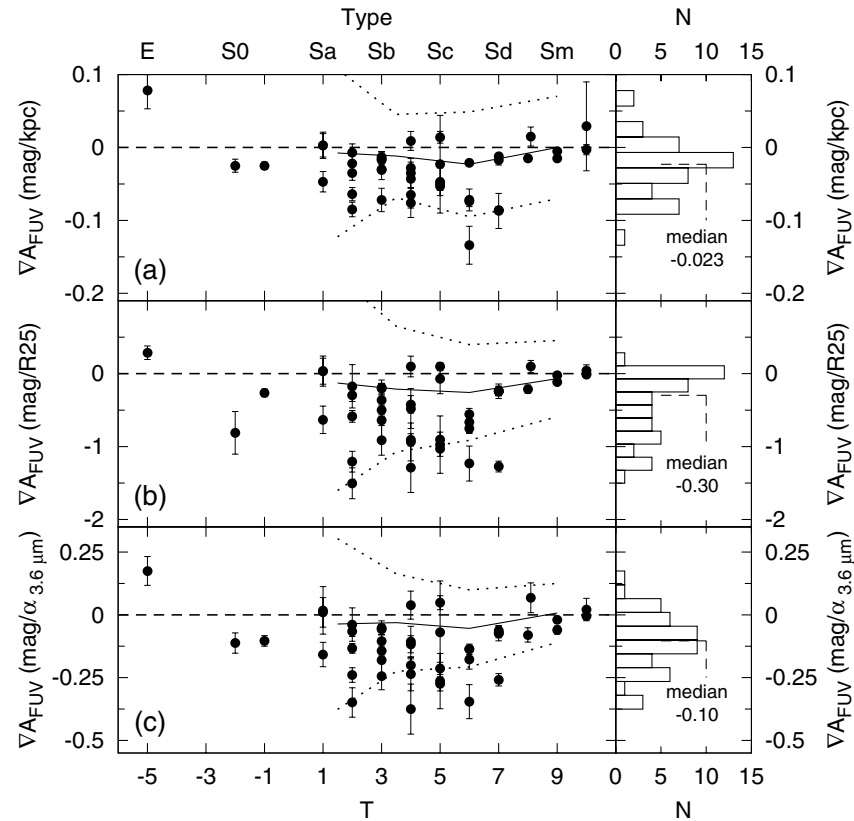

Figure 2. Radial gradients of the attenuation in the FUV, measured by applying a linear fit to the $A_{\mathrm{FUV}}$ profiles, after excluding the bulges. The gradients are expressed in mag kpc-1 (a), in units of the R25 radius (b), and in units of the radial exponential scale length of the stellar mass distribution (c). The latter was derived from the profiles measured on the degraded $3.6 \mu \mathrm{m}$ images (bulges excluded). The overall distribution of gradients for all Hubble types is shown in the histograms at the right, along with the corresponding median values. For comparison, the solid and dotted lines in each panel show the mean and $1 \sigma$ limits of the distribution of attenuation gradients derived by Muñoz-Mateos et al. (2007) for a sample of 161 nearby, face-on spirals. In that paper, we relied on the (FUV-NUV) profiles as indirect tracers of the extinction, and 2MASS $K$-band ones as a proxy for the stellar mass.

gradients are also expressed in terms of the $\mathrm{R} 25$ radius and the radial exponential scale length of the $3.6 \mu \mathrm{m}$ profiles, defined so that $I_{3.6 \mu \mathrm{m}} \propto e^{-r / \alpha_{3.6 \mu \mathrm{m}}}$. The scale length $\alpha_{3.6 \mu \mathrm{m}}$ was computed by fitting the profiles measured on the convolved $3.6 \mu \mathrm{m}$ images (again, after excluding the bulges). Since at this wavelength the luminosity traces the stellar mass, this can be considered as the stellar mass scale length. Most galaxies exhibit negative attenuation gradients, and the dispersion is larger in spirals of intermediate types.

\subsection{The IRX- $\beta$ Relation in Normal Disks}

\subsubsection{Estimating the Extinction from UV Data Alone}

In spite of the importance of correcting UV data for internal extinction, when FIR data are not available it is not possible to apply such corrections following the methods described in Section 3.1. In this regard, the slope of the UV spectrum $\beta$-or, equivalently, the (FUV-NUV) color-has been proposed as an indirect tracer of dust attenuation in starburst galaxies (Calzetti et al. 1994; Heckman et al. 1995; Meurer et al. 1995, 1999). While the infrared excess (IRX) relative to the UV seems to be tightly correlated with $\beta$ in starbursts, later studies (Bell 2002; Buat et al. 2005; Seibert et al. 2005; Cortese et al. 2006; Gil de Paz et al. 2007; Dale et al. 2007) have shown that the so-called IRX- $\beta$ relation shows rather large scatter in normal star-forming galaxies. A similar behavior has also been observed for star-forming regions within galaxies (Calzetti et al. 2005). The correlation shows a wider spread, and is also systematically shifted to redder (FUV-NUV) colors. Using radial profiles from GALEX and IRAS, Boissier et al. (2007) confirmed that the IRX $-\beta$ relation differs between starburst and normal star-forming galaxies. The relation is clearly shifted with respect to that for starburst galaxies, but the dispersion in their case seems to be reduced when using radial profiles instead of integrated photometry, possibly due to not mixing different stellar populations from the bulge and the disk.

Here we extend the work carried out in that study by deriving a new radially resolved IRX- $\beta$ diagram using data from Spitzer, which has better angular resolution than IRAS, and also probes colder dust by reaching a bit further into the FIR. An analysis of the IRX $-\beta$ plot for the SINGS galaxies, derived from integrated photometry, can be found in Dale et al. (2007).

Throughout this paper, we assume the definition of the UV spectral slope $\beta_{\mathrm{GLX}}$ given by Kong et al. (2004):

$\beta_{\mathrm{GLX}}=\frac{\log \left(f_{\lambda, \mathrm{FUV}}\right)-\log \left(f_{\lambda, \mathrm{NUV}}\right)}{\log \left(\lambda_{\mathrm{FUV}}\right)-\log \left(\lambda_{\mathrm{NUV}}\right)}=2.201(\mathrm{FUV}-\mathrm{NUV})-2$.

In Figure 3(a), we show the IRX- $\beta$ plot for each of the lowresolution radial profiles for the SINGS galaxies, classified according to their morphological type. Each data point represents a given radial bin, and those belonging to spiral galaxies are also color coded according to the (FUV-3.6 $\mu \mathrm{m}$ ) color of that bin (corrected for internal extinction), which can be used as a measure of the specific star formation rate (SFR per unit of stellar mass, sSFR hereafter) or, equivalently, the present- to pastaveraged star formation rate, usually referred to as the birthrate parameter $b$ (Scalo 1986). This color scheme cannot be applied to ellipticals, since their FUV flux is not necessarily linked to star formation (Burstein et al. 1988; O'Connell 1999; Boselli et al. 2005). As for the irregulars, the relative distribution of dust and stars is usually patchy (and not necessarily axisymmetric), so their $L_{\mathrm{TIR}} / L_{\mathrm{FUV}}$ profiles must be considered with caution. As a reference, the right vertical axis shows the extinction in the FUV derived from $L_{\mathrm{TIR}} / L_{\mathrm{FUV}}$ with the fit of Buat et al. (2005), although the color coding is based on the (FUV-3.6 $\mu \mathrm{m}$ ) color corrected for attenuation using the SFH-dependent calibration of Cortese et al. (2008). We also show the mean IRX- $\beta$ for the starburst galaxies studied by Meurer et al. (1999), as given by Kong et al. (2004), and the fit provided by Boissier et al. (2007) for the radial profiles of normal spirals.

Although differences in SFH can introduce systematic deviations in this plot (see the following section), it is desirable to get at least a rough estimate of the internal extinction for normal spirals when FIR data are not available, as has been traditionally done for starbursts. Since we are interested in obtaining a fit valid for normal star-forming spirals, we have excluded three galaxies with intense starburst activity (NGC 4536, NGC 4631, and NGC 5713). Moreover, we have only considered regions with $(\mathrm{FUV}-\mathrm{NUV})<0.9$, thus avoiding the large scatter in the TIR-to-FUV ratio at redder UV colors. We applied a nonlinear least-squares algorithm to the remaining data points, giving

$$
L_{\mathrm{TIR}} / L_{\mathrm{FUV}}=10^{0.30+1.15(\mathrm{FUV}-\mathrm{NUV})}-1.64 .
$$

The resulting fit is shown in Figure 3(a), and allows for the recovery of $\log \left(L_{\mathrm{TIR}} / L_{\mathrm{FUV}}\right)$ for normal star-forming galaxies with a residual rms uncertainty of \pm 0.27 dex. Instead of imposing a UV-color condition, we could have used the extinction-corrected (FUV-3.6 $\mu \mathrm{m})_{\text {corr }}$ color to exclude the most quiescent systems. However, such a criterion cannot be applied by an observer lacking FIR data, which are necessary to correct that color for internal extinction. While our UV condition includes a few "red" systems (in terms of their (FUV-3.6 $\mu \mathrm{m}$ ) corr $_{\text {color), they lie well }}$ within the main relation delineated by the rest of the points. The 


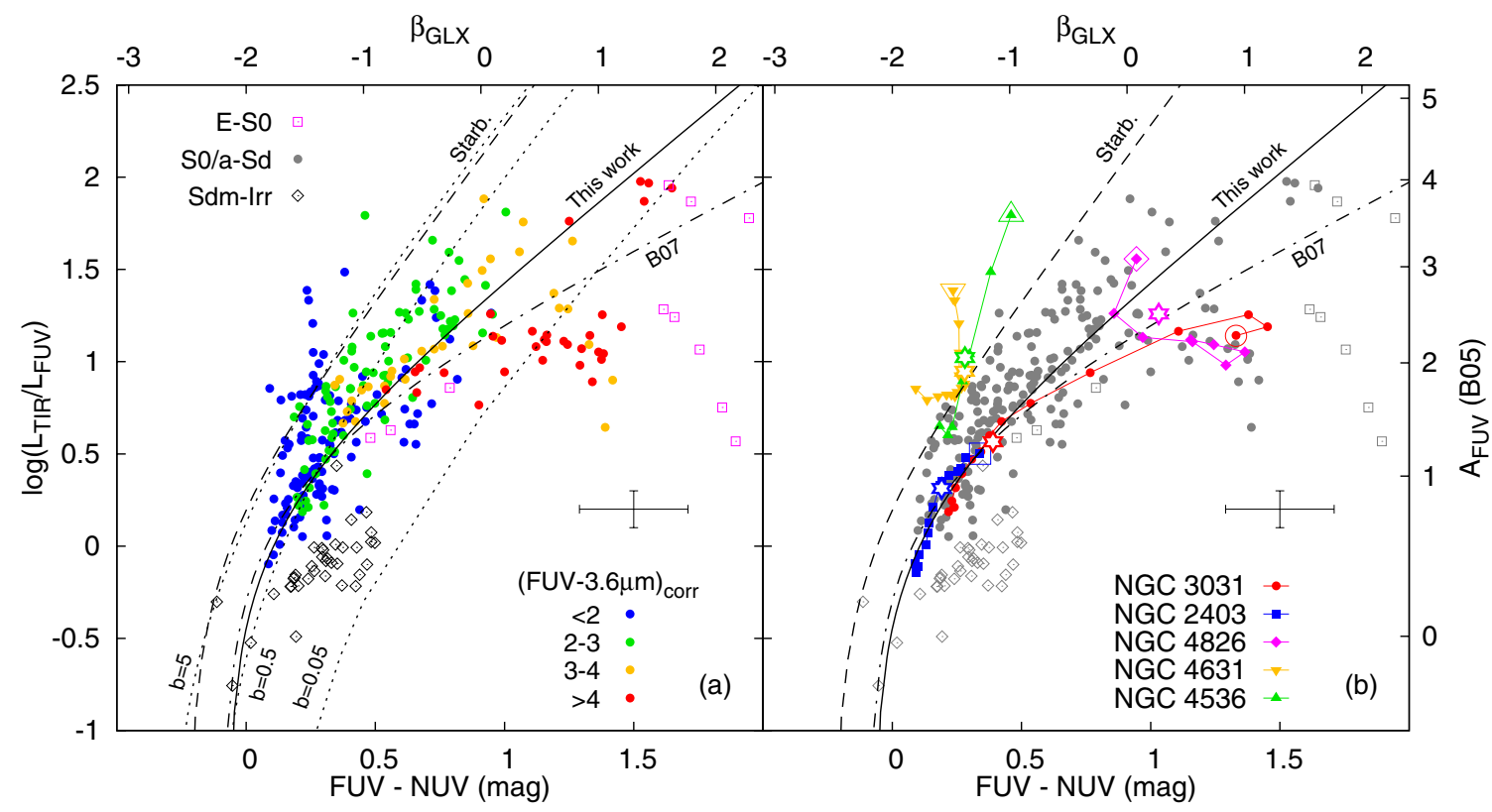

Figure 3. Ratio of the TIR-to-FUV luminosity as a function of the UV spectral slope $\left(\beta_{\mathrm{GLX}}\right)$ and the UV color, for all the profiles in the sample. The rightmost $y$-axis shows the internal extinction in the FUV for a given TIR-to-FUV ratio, according to the fits of Buat et al. (2005), only valid for late-type systems (see the text). (a) Galaxies are sorted out into different morphological types. For the disk-like galaxies, a color scheme is used to map the intrinsic (FUV-3.6 $\mu \mathrm{m})$ color, corrected for internal extinction using the age-dependent calibration of Cortese et al. (2008). The solid line corresponds to a fit to the data points bluer than (FUV-NUV) $=0.9$, excluding three starburst galaxies (see the text). The dot-dashed line shows the relation found by Boissier et al. (2007) for spiral galaxies using GALEX and IRAS data. The dashed line is the mean relation for the starburst galaxies of Meurer et al. (1999). The three dotted lines are model predictions by Kong et al. (2004) for different values of the birthrate parameter $b$. The mean error bars (including zero-point errors in all bands) are also shown. (b) Individual radial tracks followed by some galaxies in the diagram: an early-type spiral (NGC 3031), a late-type one (NGC 2403), an anemic spiral (NGC 4826; van den Bergh 1976), and two starbursts (NGC 4536 and NGC 4631, the latter being edge-on). The innermost point of each profile is marked for reference. The integrated colors of each galaxy measured by Dale et al. (2007) are shown with stars.

resulting fit is not significantly sensitive to these few points, and this selection on the observed UV color is more justified from a purely empirical point of view. Beyond (FUV-NUV) $=$ $0.9 \mathrm{mag}$, the observed dispersion is too large to reliably estimate $A_{\mathrm{FUV}}$ using this fit.

Note that our fit differs from the one derived by Boissier et al. (2007) from GALEX and IRAS data, the latter having a somewhat flatter slope at the reddest UV colors, possibly due to data points embedded within the bulges (no conditions on the UV color were imposed when computing that fit). Nevertheless, both fits are in very good agreement in the regions least affected by contamination from older stellar populations. By comparing the TIR-to-FUV profiles of the galaxies we have in common with the sample of Boissier et al. (2007), we have checked that the agreement between the TIR-to-FUV ratios is excellent. On average, our values are 0.04 dex higher, with a scatter of 0.13 dex.

In brief, in order to determine the attenuation in the FUV one must first estimate the $\log \left(L_{\mathrm{TIR}} / L_{\mathrm{FUV}}\right)$ ratio. If FIR data are available, they should be used to directly compute $\log \left(L_{\mathrm{TIR}}\right.$ using, for instance, the calibrations of Dale \& Helou (2002) or DL07. Once $\log \left(L_{\mathrm{TIR}} / L_{\mathrm{FUV}}\right)$ is known, it can be translated into $A_{\mathrm{FUV}}$ using any of the recipes available in the literature (e.g., Buat et al. 2005). In order to prevent the attenuation in early-type spirals from being overestimated, one can rely on age-dependent calibrations such as that of Cortese et al. (2008), which requires additional constraints such as optical and/or near-IR measurements. In the absence of FIR data, $A_{\mathrm{FUV}}$ can be estimated from the (FUV-NUV) color via Equation (2), but such a relation should be only employed in a statistical sense for relatively large samples of galaxies. Given the large scatter, the use of Equation (2) is discouraged for single objects.

\subsubsection{Dependence on the Star Formation History}

In Figure 3(a), only a few data points follow the starbursts relation, and they indeed correspond to galaxies with intense star formation activity (e.g., NGC 4536, NGC 4631). Most galaxies, however, lie to the right in the diagram: that is, for the same amount of attenuation, they have redder UV colors than starbursts. Differences in the SFH are the most likely explanation for this broadening (Kong et al. 2004; Calzetti et al. 2005). Besides the effects of dust, regions with more quiescent SFHs will be intrinsically redder in the UV due to their more evolved stellar populations. Indeed, there is a clear trend with the (FUV-3.6 $\mu \mathrm{m}$ ) corr color, which, as noted above, is a proxy for the present- to past-averaged star formation rate. Note that the "present" SFR, as derived from the FUV, is not instantaneous, but represents an average over the last $\sim 100$ Myr. Data points with (FUV-3.6 $\mu \mathrm{m}$ ) corr $<3$ mag follow a reasonably well-defined sequence, parallel to the starburst one; but redder regions (usually embedded within bulges) depart toward the zone populated by ellipticals.

The three dotted curves in Figure 3(a) show the IRX- $\beta$ relation predicted by Kong et al. (2004) for galaxies with different values of the birthrate parameter $b$. The comparison with (FUV-3.6 $\mu \mathrm{m})_{\text {corr }}$ should be done with caution, since the birthrate parameter of the models is the instantaneous one. The empirical relation for starbursts closely follows the model predictions for $b=5$, which falls in the range where starbursts are commonly found ( $b>2-3$; see Brinchmann et al. (2004) and references therein). The bulk of our data points are consistent with lower values of $b$. These model predictions for $A_{\mathrm{FUV}}$, however, should be considered just as average approximations, given that their uncertainties range from \pm 0.3 mag for $b \gtrsim 0.3$ 


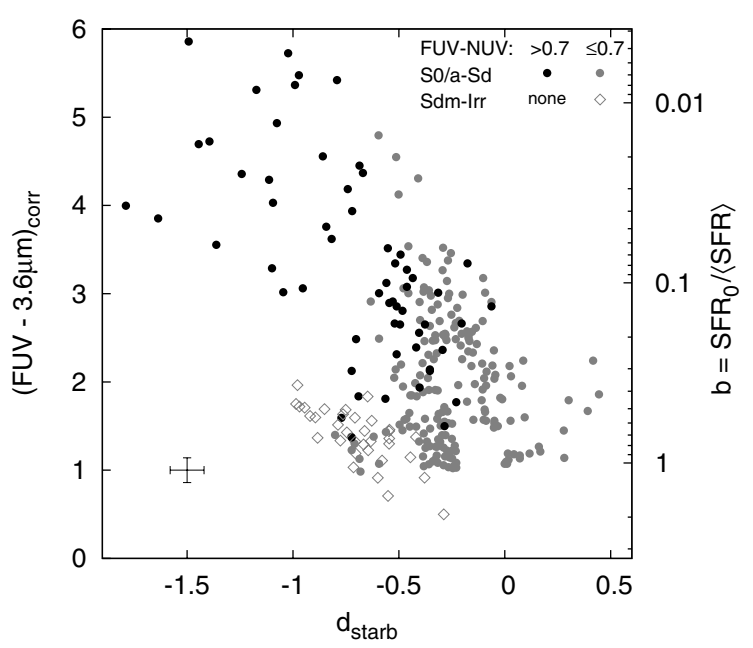

Figure 4. Relation between the intrinsic (FUV-3.6 $\mu \mathrm{m}$ ) color (i.e., corrected for internal extinction) and the perpendicular distance to the mean relation for starbursts in the IRX- $\beta$ diagram (see, Figure 3). Negative distances correspond to data points below the starbursts curve. Different symbols and colors are used depending on the Hubble type and the observed UV color.

to \pm 1 mag for lower values, owing to differences in the particular details of the SFH and the dust content (Kong et al. 2004).

We further explore this trend in Figure 4, where we have plotted the (FUV-3.6 $\mu \mathrm{m})_{\text {corr }}$ color as a function of the perpendicular (i.e., shortest) distance from each data point to the starburst relation, $d_{\text {starb }}$ (see Kong et al. 2004). Although with considerable dispersion, the trend is rather evident: regions with intrinsically redder (FUV-3.6 $\mu \mathrm{m})_{\text {corr }}$ colors-having then lower current-to-past star formation activity - are clearly located further away from the relation for starbursts. Interestingly, selection effects can blur or even make this trend vanish. For instance, if we only consider regions bluer than (FUV-NUV) $\sim 0.7$, no clear correlation between SFH and $d_{\text {starb }}$ can be inferred. Therefore, one needs to explore a wide range in SFHs in order to see this trend. This could imply that although SFH seems to be driving this departure from the starburst relation, other factors such as dust geometry or the extinction law might be also contributing in different ways. It should be noted as well that if we choose another IRX $-\beta$ curve as a reference instead of the starburst one (i.e., MW-type dust with different geometries, for instance), all perpendicular distances will change accordingly.

This could explain the different conclusions reached by authors studying integrated properties of galaxies. Kong et al. (2004) found a correlation between $d_{\text {starb }}$ and different indicators of the SFH, such as the $D_{n}(4000)$ break and the $\mathrm{H} \alpha$ equivalent width (EW). Cortese et al. (2006) estimated the birthrate parameter from $\mathrm{H} \alpha$ - and $H$-band luminosities for an optically selected sample of normal star-forming galaxies, and found a weak correlation between $b$ and $d_{\text {starb }}$, with considerable scatter. The fact that their galaxies belong to nearby clusters might be in part responsible for the observed scatter. Interactions with the intracluster medium and the cluster potential well may likely affect their SFHs by removing gas from the disks and quenching their SF activity, thus progressively turning them into anemic spirals (see, e.g., Boselli \& Gavazzi 2006 and references therein). In their analysis of the integrated properties of the SINGS galaxies, Dale et al. (2007) found that most of the scatter toward redder UV colors in the IRX- $\beta$ diagram was due to ellipticals and early-type spirals. Panuzzo et al. (2007) studied a UV-selected sample of galaxies, and did not find any systematic deviation from the starburst relation that depended on $b$, computed from NUV- and $H$-band luminosities. However, their UV-selected sample did not contain objects redder than $(\mathrm{FUV}-\mathrm{NUV}) \sim 0.7$, thus making it difficult to infer any correlation, as Figure 4 demonstrates.

In order to further illustrate these issues, in Figure 3(b) we have highlighted some tracks for particular galaxies, connecting the data points of annular regions at different galactocentric distances within each particular galaxy. The innermost point of each profile is marked for clarity, and the integrated colors of each galaxy are shown with open stars. "Normal" spirals follow their own IRX $-\beta$ relation, which is quite similar in shape to the average one (modulo global offsets in the overall UV color and/or extinction). In early-type spirals with well-defined bulges, such as NGC 3031 (M 81), the track clearly deviates toward redder colors as we move closer to the center, due to the contribution of more evolved stars in the bulge. This does not happen in late-type galaxies, like NGC 2403, given their smaller bulge-to-disk ratios, especially at UV wavelengths.

The SINGS sample also includes some peculiar objects that do not follow these smooth trends. This is the case of NGC 4826, which is a clear example of an anemic spiral (van den Bergh 1976). Although it has a global radial extent of $\sim 13 \mathrm{kpc}$ at $3.6 \mu \mathrm{m}$, the bulk of the star formation activity — as traced by the FUV or $24 \mu \mathrm{m}$ images, for instance-seems to be restricted to the central $5 \mathrm{kpc}$. Therefore, as we move away from the center toward the outer and more quiescent regions, the track followed by this galaxy in the IRX $-\beta$ diagram heads toward the region populated by bulges. Something similar happens with NGC 4569, another anemic galaxy in the Virgo cluster, where ram pressure stripping seems to have quenched the star formation in the outer regions (Boselli et al. 2006).

Systems hosting starburst activity, like NGC 4536, lie close to the empirical relation given by Kong et al. (2004). In very edge-on systems like NGC 4631 (which also happens to host starburst activity), geometry might also play an important role. The observed TIR-to-FUV ratio is probably larger than the one we would measure if the galaxy was face-on (besides the fact that this galaxy is probably more dust-rich than the average spiral galaxy). However, we do not find any significant trend between the position in the IRX- $\beta$ diagram and inclination, apart from this extreme case. At fixed metallicity, however, inclination does seem to play a role (see Section 3.3).

Kong et al. (2004) suggest that galaxies in their sample departing too much from the starburst relation require very quiescent SFHs, with very short timescales of star formation, not typical of spirals. They invoke an additional mechanism that can contribute to broadening the IRX $-\beta$ relation, consisting of an extra burst of star formation at some point during the galaxy's lifetime, superimposed on top of an otherwise smooth SFH. While this can certainly add more scatter, we note that in our radial analysis most regions lying at large distances from the starburst relation are either bulges or regions of anemic spirals with clear signs of star formation quenching. Indeed, there are 18 galaxies in our sample having points with $(\text { FUV-3.6 } \mu \mathrm{m})_{\text {corr }}>3$. Five of them are ellipticals or lenticulars, and 11 are early-type spirals (S0/a-Sb), including the already mentioned anemic ones. Therefore, at least for these very quiescent systems, a rapidly declining SFH might be a more reasonable possibility.

From the above analysis, we can conclude that the SFH is possibly driving the departure of star-forming regions from the locus of starburst galaxies in the IRX $-\beta$ diagram. However, the trends with different tracers of SFH (both here and in other 


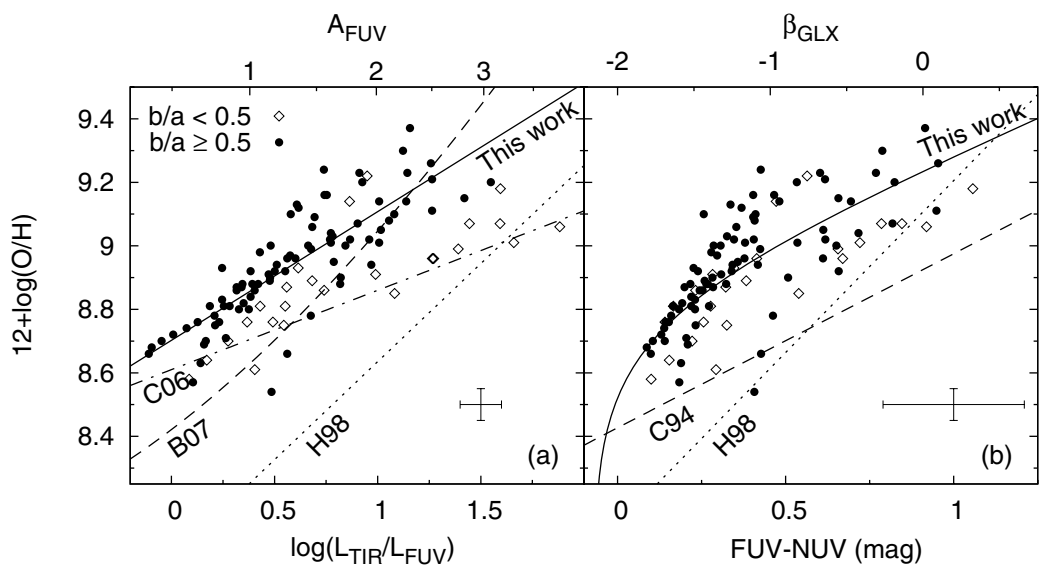

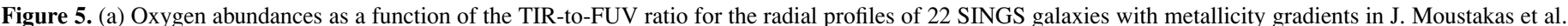

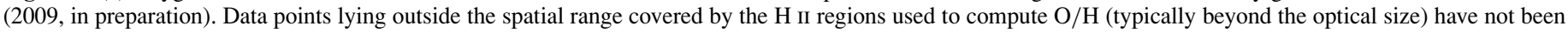

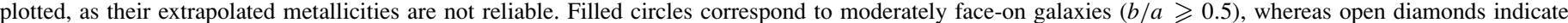

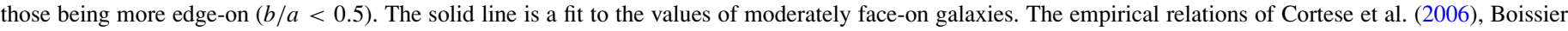

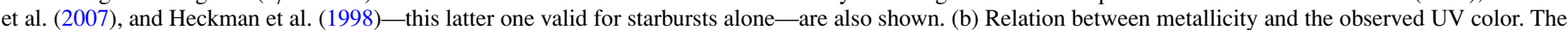

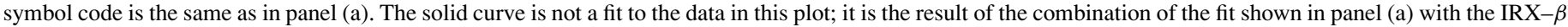

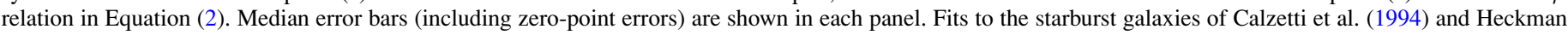
et al. (1998) are also plotted.

studies) seem to be quite noisy and not always evident- they can actually disappear if the range of explored SFHs is not wide enough. Several reasons might explain this. First of all, observational errors might blur the offset between regions if their SFHs are not different enough. Secondly, realistic SFHs cannot be parameterized with a single quantity like $b$; indeed, the intermediate bursts proposed by Kong et al. (2004) were shown to contribute to the observed scatter. Besides, a number of additional factors such as the relative geometry of dust and stars, the shape of the internal extinction law and the IMF can also have a great impact (Burgarella et al. 2005; Panuzzo et al. 2007).

\subsection{Influence of Metallicity and Inclination}

Attenuation and metallicity are known to be correlated in starburst galaxies (Calzetti et al. 1994; Heckman et al. 1998), which can be interpreted in terms of increasing extinction at larger dust-to-gas ratios (see the discussion and references given in Section 4.3.6). Such a trend has been also observed in normal star-forming galaxies, both with integrated data (see, e.g., Cortese et al. 2006) and with radial profiles (Boissier et al. 2007). These studies find an offset with respect to the starbursts relation, with normal galaxies being less attenuated at a given metallicity.

In Figure 5(a), we study the radial trend between metallicity and the TIR-to-FUV ratio. Metallicity gradients for 22 SINGS galaxies were taken from the compilation of J. Moustakas et al. (2009, in preparation). These authors compute the oxygen abundances using two different calibrations: Kobulnicky \& Kewley (2004) and Pilyugin \& Thuan (2005). The Kobulnicky \& Kewley (2004) values tend to be smaller than the Pilyugin \& Thuan (2005) by 0.6 dex, on average. However, to first order the radial abundance gradients derived for these 22 SINGS galaxies are independent of the adopted calibration. In this paper, we adopt the oxygen abundances derived with the Kobulnicky \& Kewley (2004) calibration, and refer the reader to J. Moustakas et al. (2009, in preparation) for more details on this subject.

Although our photometric profiles extend up to $\sim 1.5$ times the optical size of each galaxy, the $\mathrm{H}$ II regions where oxygen abundances were measured do not usually reach beyond the optical radius, and are also missing in the central regions of some galaxies. In order to avoid unsafe extrapolations of the metallicity values, we only plot those data points lying within the radial range determined by the innermost and outermost abundance measurements in each galaxy. The empirical fits of Cortese et al. (2006) for cluster galaxies and of Boissier et al. (2007) for nearby spirals are shown for reference, as well as the one of Heckman et al. (1998) for starburst galaxies.

We confirm that the most metal-rich regions also suffer from larger attenuation. Despite the scatter, we can see that at fixed metallicity the more inclined galaxies appear to have larger TIRto-FUV ratios: from our perspective, UV photons must travel through larger amounts of dust before reaching us than if the galaxy was to be observed face-on. We performed a linear fit to those points with $b / a \geqslant 0.5$, resulting in

$$
12+\log (\mathrm{O} / \mathrm{H})=8.70+0.405 \log \left(L_{\mathrm{TIR}} / L_{\mathrm{FUV}}\right) .
$$

In Section 3.2.2, we stated that no significant trend with inclination can be detected in the IRX- $\beta$ diagram, yet the TIRto-FUV ratio is clearly larger for nearly edge-on galaxies for a given metallicity. This apparent riddle is solved in Figure 5(b), where we plot the metallicity as a function of the (FUV-NUV) color for the same elliptical annuli as in panel (a). Both magnitudes are clearly correlated, and inclination also plays an important role here, since the observed UV color is redder at larger inclinations for a given metallicity. The effect of inclination depicted in panels (a) and (b) is such that more inclined galaxies appear redder in the UV, but also have larger TIR-to-FUV ratios. As a consequence, decreasing $b / a$ will not significantly displace the corresponding data points out of the main trend seen in Figure 3, but would move them along it.

Since the TIR-to-FUV ratio seems to be linearly correlated with metallicity for face-on galaxies, the distribution of filled circles in Figure 5(b) is analogous to the IRX $-\beta$ plot. The solid line in that panel is obtained by combining Equations (3) and (2), not from a direct fit to the data in the plot.

\section{COMPARISON WITH PHYSICAL DUST MODELS}

\subsection{Description of the Models}

In order to derive the physical properties of the dust beyond mere dust attenuation, we need to assume a given model for the 
chemical composition and size distribution of the dust grains, and take into account their spatial distribution relative to the heating stars. Modeling the spatial distribution of cold and warm dust, relative to stars, has been the subject of much attention, especially in edge-on galaxies (see, e.g., Xilouris et al. 1999; Popescu et al. 2000). In this paper, we will employ the dust models of DL07, which constitute an update of those developed by Weingartner \& Draine (2001) and Li \& Draine (2001). These models were successfully applied to the integrated photometry of the SINGS galaxies (Draine et al. 2007, D07 hereafter). The reader is referred to D07 and DL07 for a more detailed description than is provided for context in the following paragraphs.

The DL07 models describe the interstellar dust as a mixture of carbonaceous grains and amorphous silicate grains, whose size distributions are chosen to mimic the observed extinction law in the Milky Way (MW). Although other size distributions can be chosen to reproduce the extinction curves in the Large and Small Magellanic Clouds, D07 showed that they did not provide significantly better fits than the MW ones for the SINGS galaxies.

The physical properties of the smallest carbonaceous grains in the models are those of PAH particles. The PAH abundance is characterized by the PAH index, $q_{\mathrm{PAH}}$, defined as the fraction of the dust mass in the form of PAH grains with less than $10^{3}$ carbon atoms. Models with different PAH abundances can be generated by changing the size distribution of the dust grains while keeping constant the overall average extinction. The fraction of neutral and ionized PAHs is the one estimated by Li \& Draine (2001) for the diffuse ISM in the MW. The resulting spectra have fixed band ratios in the IRAC bands, but those values are representative of normal spiral galaxies.

One also has to make several assumptions regarding the starlight heating the dust. In the DL07 models, the specific energy density of the starlight is set to be a scaled version of the local interstellar radiation field of the MW estimated by Mathis et al. (1983). The intensity of the incident starlight is then characterized by a dimensionless scale factor $U$.

Not all the dust within a galaxy is exposed to the same range of starlight intensities $U$, and the emitted SED will result from the superposition of the SEDs associated with the diffuse ISM, starforming regions, etc. Dale et al. (2001) proposed a power-law description of the dust heating, and used this model to describe the global IR SEDs of the SINGS galaxies in Dale et al. (2007).

The DL07 models follow a slightly different approach. The majority of the dust is supposed to be located in the diffuse ISM, being heated by a radiation field with a constant intensity $U_{\min }$. A smaller fraction $\gamma$ of the dust is exposed to starlight with intensities ranging from $U_{\min }$ to $U_{\max }$, following the power-law expression mentioned above. This term is intended to represent the dust enclosed in photodissociation regions (PDRs), where the radiation field is much more intense than in the diffuse component. Therefore, the amount of dust $d M_{\text {dust }}$ exposed to radiation intensities between $U$ and $U+d U$ can be expressed as

$$
\frac{d M_{\text {dust }}}{d U}=(1-\gamma) M_{\text {dust }} \delta\left(U-U_{\min }\right)+\gamma M_{\text {dust }} \frac{\alpha-1}{U_{\min }^{1-\alpha}-U_{\max }^{1-\alpha}} U^{-\alpha} .
$$

D07 find that the precise value of $\alpha$ does not have a great impact on the quality of the fits, given that the relative contribution of the diffuse and PDR components can be now parameterized with $\gamma$, and suggest fixing $\alpha=2$, which works well for a wide range of galaxy properties. They also find that no particular value of $U_{\max }$ seems to be favored, and hence adopt a fixed value of $U_{\max }=10^{6}$. In addition, they suggest using $0.7 \leqslant U_{\min } \leqslant 25$ when submillimeter data are not available, since smaller values correspond to dust temperatures below $\sim 15 \mathrm{~K}$, which cannot be probed with MIPS photometry alone. This lower cutoff for $U$ prevents the fitting technique from invoking larger amounts of cold dust heated by weak starlight with very small values of $U_{\min }$, although this would underestimate the total dust mass if large amounts of cold dust are actually present. Note, however, that for those SINGS galaxies with available submillimeter data, D07 concluded that omitting these data does not seem to introduce any evident systematic offset in the derived total dust masses, although the scatter in the resulting dust masses may increase up to $\sim 50 \%$.

For a given PAH abundance, the emitted SED results from the linear combination of the SEDs associated with the diffuse and PDR component. Therefore, the shape of the SED is controlled by three parameters: $q_{\mathrm{PAH}}, U_{\min }$, and $\gamma$, and the dust mass surface density can be derived when normalizing the SED.

Besides the models of DL07, the SINGS galaxies have been used as a benchmark for other different dust models. For instance, da Cunha et al. (2008) developed a simple model to interpret the mid- and far-IR SEDs of galaxies consistently with the emission in the UV, optical, and near-IR. While their prescriptions for the dust emission are simpler and more empirical than those of DL07, by linking the absorption of starlight from the UV to the near-IR with dust emission at midand far-IR, they are able to extract the properties of both stars and dust in a consistent way. The dust properties they derived for the SINGS galaxies are in good agreement with those presented in D07.

\subsection{Fitting Procedure}

Using our IRAC and MIPS radial profiles, we can therefore estimate $q_{\mathrm{PAH}}, U_{\min }, \gamma$, and the dust mass surface density $\Sigma_{M_{\text {dust }}}$ as a function of the galactocentric distance, by finding the bestfit model for the SED within each elliptical annulus. Following DL07, by taking linear interpolations of the original models we built a grid of models with different PAH abundances (ranging from $q_{\mathrm{PAH}}=0.4 \%$ to $4.6 \%$ in steps of $0.1 \%$ ) and values of $\gamma$ (from $0 \%$ to $30 \%$ in steps of $0.1 \%$ ). We looked for the best-fit model by minimizing the reduced $\chi^{2}$, computed over the IRAC $5.8 \mu \mathrm{m}$ and $8.0 \mu \mathrm{m}$ channels and the three MIPS bands, after correcting them for stellar emission (see below). We used the quadratic sum of the photometric uncertainties and the zeropoint errors as weights, plus an additional $\sim 10 \%$ error at each band to account for the limited accuracy of the model, following D07. The $1 \sigma$ uncertainty for each parameter was derived by projecting the overall $\chi^{2}$ distribution over the one-dimensional space of that parameter, and then looking for the values which satisfied $\chi^{2}=\chi_{\min }^{2}+1$ (Press et al. 1992).

The DL07 models provide the multi-wavelength flux densities emitted by the dust components. Since the IRAC $3.6 \mu \mathrm{m}$ and $4.5 \mu \mathrm{m}$ bands are almost entirely dominated by stellar emission (see, e.g., Pérez-González et al. 2006), we did not take them explicitly into account when performing the fits; however, we used the $3.6 \mu \mathrm{m}$ fluxes to estimate the stellar fluxes at the other bands. We used the stellar populations synthesis models of Bruzual \& Charlot (2003) to compute the flux ratios $\left\langle F_{\nu}\right\rangle_{\lambda} /\left\langle F_{v}\right\rangle_{3.6}$. For a fixed age of $13 \mathrm{Gyr}$, and assuming exponential SFHs with timescales $\tau$ ranging from 0.01 to $20 \mathrm{Gyr}$, we obtained $\left\langle F_{\nu}\right\rangle_{\lambda} /\left\langle F_{\nu}\right\rangle_{3.6}=0.660,0.453,0.269$, and 0.032 for $\lambda=4.5,5.8,8.0$, and $24 \mu \mathrm{m}$, respectively, assuming solar 


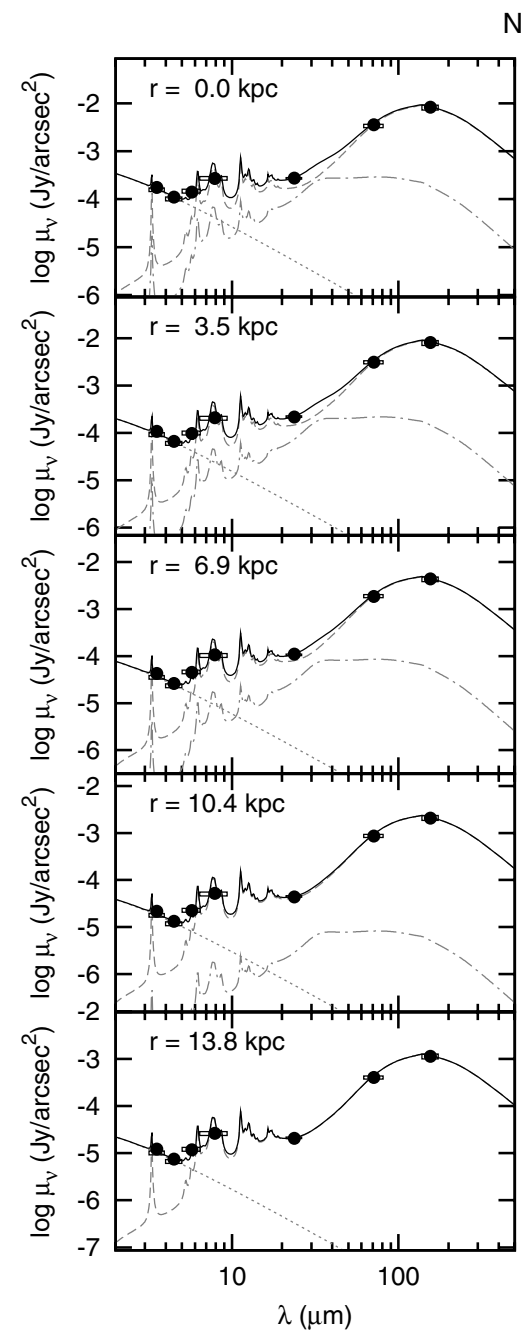

NGC 7331

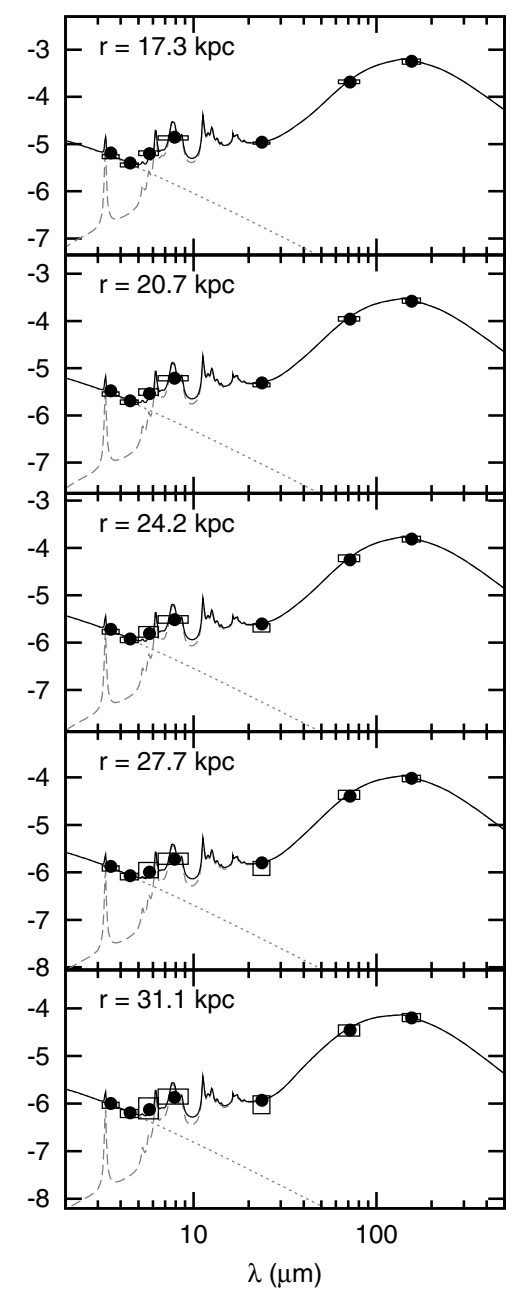

Figure 6. Observed SEDs and model spectra at different galactocentric distances, without correcting for inclination. A sample plot for NGC 7331 is shown; additional figures for the remaining galaxies can be found in the electronic version of the journal. The observed flux densities are marked with rectangular boxes, their width showing the corresponding bandpass and their height showing the observational errors. The best-fitting model spectra (solid line) results from the combination of a diffuse component (dashed line), the emission from hot dust (dash-dotted line), and the stellar emission (dotted line). The filled circles correspond to the model spectra convolved with each bandpass.

(A complete figure set (64 images) is available in the online journal.)

metallicity. These values are close to the ones derived from a $5000 \mathrm{~K}$ blackbody SED, and depend very weakly on $\tau$, with variations of less than $5 \%$ over the range of extinction-corrected (FUV-3.6 $\mu \mathrm{m}$ ) colors of our data. Larger deviations can be seen for supersolar metallicities, and also for very young populations (age $\lesssim 1 \mathrm{Gyr}$ ), due to the contribution of AGB stars. However, the prescription given above yields good fits for our SEDs.

To check the possible systematic effects introduced by the stellar emission correction, we fitted the models twice for all galaxies, using our flux ratios and the ones estimated by Helou et al. (2004) using stellar population modeling from Starburst99 (Leitherer et al. 1999). Their scaling factors are somewhat bluer than ours; hence the derived PAH abundances are systematically larger, since the estimated stellar emission at the IRAC bands is smaller. Nevertheless, the median offset in the derived abundances is only $\sim 5 \%-10 \%$. There is, however, a much larger difference in those regions where the PAH contribution to the $8 \mu \mathrm{m}$ flux density is less than half of the stellar one at that band (e.g., in bulges). The values of $q_{\mathrm{PAH}}$ might then be unreliable in these cases, so we have excluded them from our analysis. As for the fraction $\gamma$ of the dust mass exposed to highintensity radiation fields, for $\gamma \lesssim 1 \%$ the resulting values using
Starburst99 are $\sim 10 \%$ smaller than the ones obtained when using the factors from the models of Bruzual \& Charlot (2003), with no difference at larger values. Finally, the dust mass and luminosity surface densities, as well as the radiation field scale factor $U$, do not vary noticeably. This is not surprising, since these parameters are mostly determined by the MIPS fluxes, where the stellar contamination is negligible. For the remainder of the analysis described here, we adopt the factors obtained from the models of Bruzual \& Charlot (2003).

Further systematic effects might result from using a small number of bands when fitting the models. In Appendix B, we discuss this issue in detail, and demonstrate that our results are consistent with the global values obtained by D07 using a more complete set of IR data.

\subsection{Results from the Model}

\subsubsection{Individual Profiles and SEDs}

In Figure 6, we show the IR SEDs over a range of galactocentric distances, along with the best-fitting models, for one of the sample galaxies: NGC 7331 (see the online edition for a complete figure set for the whole sample). The correspond- 
ngc7331
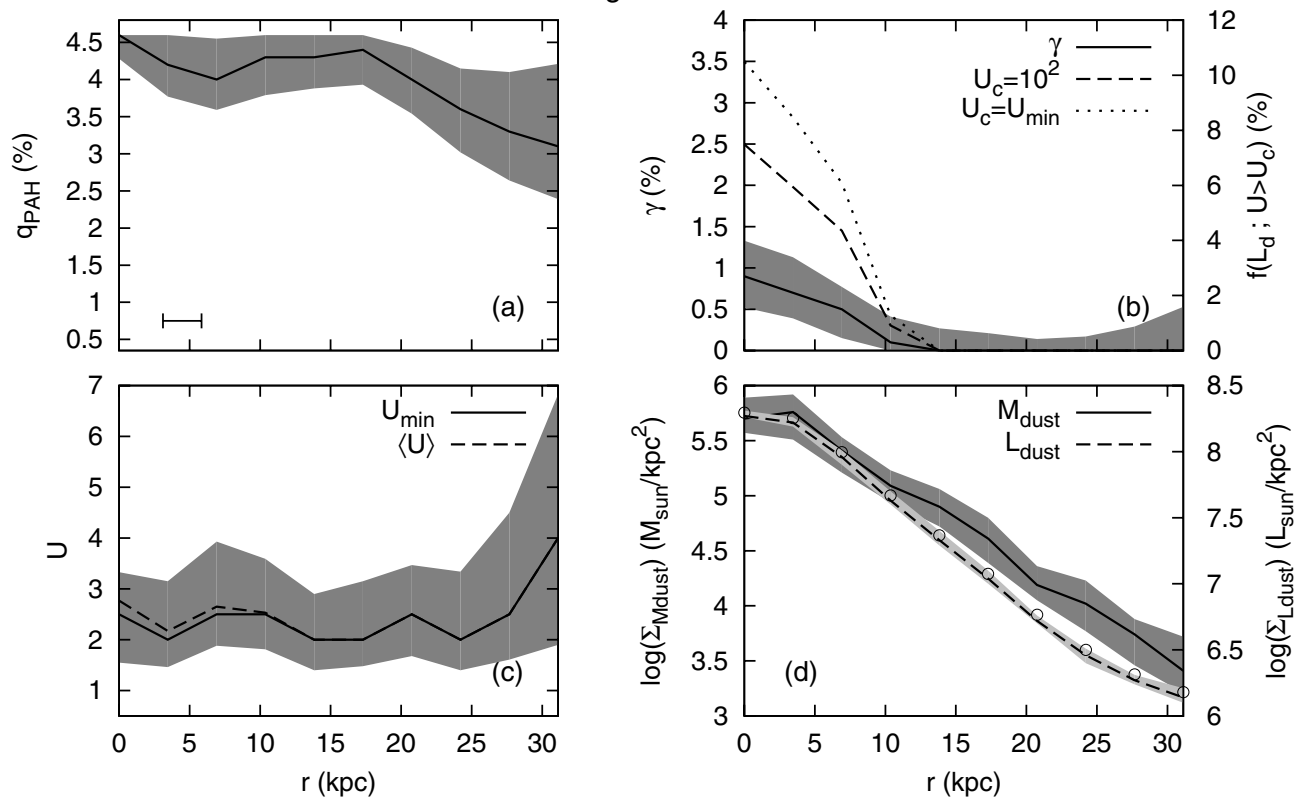

Figure 7. Radial distribution of the different parameters derived from the dust models. A sample plot is shown for NGC 7331; additional figures for the remaining galaxies can be found in the electronic version of the journal. The different lines show the best-fitting values at each radius, and the gray bands show the estimated uncertainties. (a) Fraction of the dust mass in the form of PAHs. Regions in which the stellar emission at $8 \mu \mathrm{m}$ is more than twice the one from PAHs are marked (when present) with a dotted line instead of a solid one. The FWHM of the MIPS $160 \mu \mathrm{m}$ band (38 $)$ is marked with a horizontal segment along the major axis. (b) Fraction $\gamma$ of the total dust mass heated by very intense starlight. The right vertical axis shows the fraction of the dust luminosity contributed by regions exposed to radiation fields with $U>U_{\min }$ and $U>10^{2}$ (their errors are not shown for clarity). (c) Scale factor of the minimum $\left(U_{\min }\right)$ and average ( $\left.\langle U\rangle\right)$ starlight intensity heating the dust, in units of the local MW radiation field. The errors of $\langle U\rangle$ are not shown. (d) Dust mass (left) and luminosity (right) surface densities, both corrected for inclination. The open circles show the dust luminosity profiles obtained with the photometric estimator of DL07.

(A complete figure set (53 images) is available in the online journal.)

Table 4

Model Parameters Profiles

\begin{tabular}{|c|c|c|c|c|c|c|c|c|c|}
\hline $\begin{array}{c}r \\
(\operatorname{arcsec}) \\
(1)\end{array}$ & $\begin{array}{c}r \\
(\mathrm{kpc}) \\
(2)\end{array}$ & $\begin{array}{c}q_{\mathrm{PAH}} \\
(\%) \\
(3)\end{array}$ & $\begin{array}{c}\gamma \\
(\%) \\
(4)\end{array}$ & $\begin{array}{c}f\left(U>10^{2}\right) \\
(\%) \\
(5)\end{array}$ & $\begin{array}{c}U_{\min } \\
(6) \\
\end{array}$ & $\begin{array}{l}\langle U\rangle \\
(7)\end{array}$ & $\begin{array}{c}\log \Sigma_{L_{\text {dust }}} \\
\left(L_{\odot} \mathrm{kpc}^{-2}\right) \\
(8)\end{array}$ & $\begin{array}{c}\log \Sigma_{M_{\text {dust }}} \\
\left(M_{\odot} \mathrm{kpc}^{-2}\right) \\
(9)\end{array}$ & $\begin{array}{c}\log \left(M_{\text {dust }} / M_{\text {gas }}\right) \\
\text { (10) }\end{array}$ \\
\hline \multicolumn{10}{|l|}{ NGC 7331} \\
\hline 0 & 0.0 & $4.6_{-0.3}^{+0.0}$ & $0.9_{-0.4}^{+0.4}$ & $7.5_{-6.3}^{+4.3}$ & $2.5_{-0.9}^{+0.8}$ & $2.8_{-1.1}^{+0.7}$ & $8.27_{-0.01}^{+0.04}$ & $5.70_{-0.13}^{+0.19}$ & $-1.55_{-0.13}^{+0.19}$ \\
\hline 48 & 3.5 & $4.2_{-0.4}^{+0.4}$ & $0.7_{-0.3}^{+0.4}$ & $5.9_{-5.9}^{+5.2}$ & $2.0_{-0.5}^{+1.1}$ & $2.2_{-0.6}^{+1.2}$ & $8.22_{-0.03}^{+0.04}$ & $5.76_{-0.25}^{+0.16}$ & $-1.88_{-0.25}^{+0.16}$ \\
\hline 96 & 6.9 & $4.0_{-0.4}^{+0.6}$ & $0.5_{-0.3}^{+0.3}$ & $4.3_{-4.3}^{+6.2}$ & $2.5_{-0.6}^{+1.4}$ & $2.6_{-0.8}^{+1.3}$ & $7.96_{-0.06}^{+0.05}$ & $5.41_{-0.20}^{+0.12}$ & $-1.94_{-0.20}^{+0.12}$ \\
\hline 144 & 10.4 & $4.3_{-0.5}^{+0.3}$ & $0.1_{-0.1}^{+0.3}$ & $0.9_{-0.9}^{+2.9}$ & $2.5_{-0.7}^{+1.1}$ & $2.5_{-0.7}^{+1.1}$ & $7.63_{-0.03}^{+0.07}$ & $5.09_{-0.15}^{+0.14}$ & $-2.00_{-0.16}^{+0.15}$ \\
\hline 192 & 13.8 & $4.3_{-0.4}^{+0.3}$ & $0.0_{-0.0}^{+0.3}$ & $0.0_{-0.0}^{+2.4}$ & $2.0_{-0.6}^{+0.9}$ & $2.0_{-0.5}^{+1.2}$ & $7.33_{-0.04}^{+0.07}$ & $4.90_{-0.18}^{+0.16}$ & $-2.09_{-0.20}^{+0.18}$ \\
\hline 240 & 17.3 & $4.4_{-0.5}^{+0.2}$ & $0.0_{-0.0}^{+0.2}$ & $0.0_{-0.0}^{+2.0}$ & $2.0_{-0.5}^{+1.1}$ & $2.0_{-0.4}^{+1.3}$ & $7.04_{-0.04}^{+0.06}$ & $4.61_{-0.22}^{+0.19}$ & $-2.23_{-0.27}^{+0.25}$ \\
\hline 288 & 20.7 & $4.0_{-0.5}^{+0.4}$ & $0.0_{-0.0}^{+0.1}$ & $0.0_{-0.0}^{+1.6}$ & $2.5_{-0.8}^{+1.0}$ & $2.5_{-0.8}^{+1.1}$ & $6.72_{-0.02}^{+0.04}$ & $4.19_{-0.14}^{+0.17}$ & $-2.39_{-0.27}^{+0.29}$ \\
\hline 336 & 24.2 & $3.6_{-0.6}^{+0.6}$ & $0.0_{-0.0}^{+0.2}$ & $0.0_{-0.0}^{+2.4}$ & $2.0_{-0.6}^{+1.3}$ & $2.0_{-0.6}^{+1.5}$ & $6.46_{-0.06}^{+0.05}$ & $4.02_{-0.25}^{+0.21}$ & $-2.23_{-0.35}^{+0.33}$ \\
\hline 384 & 27.7 & $3.3_{-0.7}^{+0.8}$ & $0.0_{-0.0}^{+0.3}$ & $0.0_{-0.0}^{+4.4}$ & $2.5_{-0.9}^{+2.0}$ & $2.5_{-0.8}^{+2.0}$ & $6.27_{-0.03}^{+0.04}$ & $3.74_{-0.28}^{+0.14}$ & $-2.21_{-0.38}^{+0.30}$ \\
\hline 432 & 31.1 & $3.1_{-0.7}^{+1.1}$ & $0.0_{-0.0}^{+0.5}$ & $0.0_{-0.0}^{+8.6}$ & $4.0_{-2.1}^{+2.8}$ & $4.0_{-2.1}^{+1.3}$ & $6.14_{-0.04}^{+0.07}$ & $3.41_{-0.20}^{+0.31}$ & $-2.26_{-0.38}^{+0.45}$ \\
\hline
\end{tabular}

Notes. Radial profiles of the model parameters. (1) Radius along the semimajor axis in arcsec. (2) Radius along the semimajor axis in kpc. (3) Fraction of the dust mass in the form of PAHs. This value is not reliable in regions marked with a dagger ( $\dagger$ ), where the dust contribution to the observed $8 \mu \mathrm{m}$ flux is lees than half of the stellar emission at that band. (4) Fraction of the dust mass heated by very intense starlight. (5) Fraction of the dust luminosity contributed by regions heated by starlight with $U>10^{2}$. (6) Minimum value for the starlight scale factor, in units of the local MW radiation field. (7) Dust-weighted average scale factor for the starlight intensity. (8) Dust luminosity surface density, corrected for inclination. (9) Dust mass surface density, corrected for inclination. (10) Dust-to-gas ratio. The dust mass is computed as $M_{\text {gas }}=1.36 \times\left(M_{\mathrm{HI}}+M_{\mathrm{H}_{2}}\right)$, or $M_{\text {gas }}=1.36 \times M_{\mathrm{HI}}$ when CO data are not available. In the latter case, the values are marked with a double dagger $(* * *)$, and should be treated as upper limits (at least in the inner regions).

(This table is available in its entirety in a machine-readable form in the online journal. A portion is shown here for guidance regarding its form and content.)

ing numerical values of the model parameters are quoted in Table 4, which is shown in its entirety in the online edition. Note that the results for NGC 7331 can be readily compared with those from Thilker et al. (2007). As explained above, each SED can be decomposed in two terms: a "diffuse" component (dashed line), heated by a constant radiation field with $U=U_{\min }$, and a PDR one (dash-dotted line), with $U_{\min }<U<U_{\max }$. The latter is particularly important to account for the observed flux 


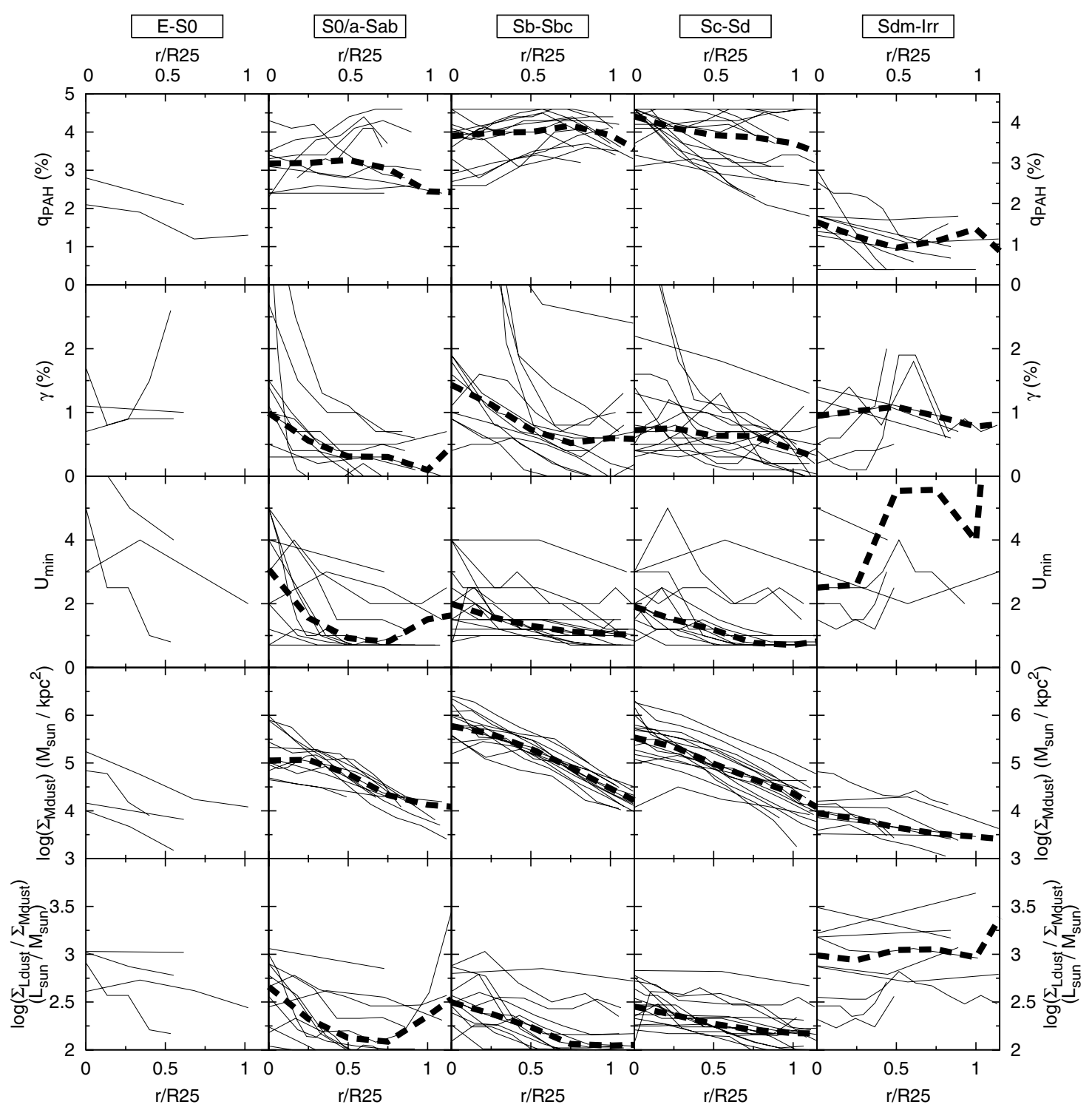

Figure 8. Radial profiles of the different parameters involved in the dust models. Galaxies have been grouped according to their morphological type. The different parameters are the PAH abundance $\left(q_{\mathrm{PAH}}\right)$, the fraction of the dust mass exposed to intense radiation fields $(\gamma)$, the intensity of the "diffuse" heating starlight, in units of the local MW radiation field $\left(U_{\min }\right)$, the de-projected dust mass surface density $\left(\Sigma_{M_{\text {dust }}}\right)$, and the dust light-to-mass ratio $\left(L_{\text {dust }} / M_{\text {dust }}\right)$. The radial coordinate is normalized to the optical size of each galaxy. The thick dashed lines show the median profiles in each panel. No median profiles are shown for ellipticals and lenticulars, due to the small number of galaxies in that bin. The median $U_{\min }$ profile for Sdm-Im galaxies is above most of the profiles displayed in that panel due to some galaxies having $U_{\min }>5$, which are not shown here for clarity.

density at $24 \mu \mathrm{m}$. The total resulting SED is shown with a solid line, and includes not only the emission contributed by dust, but also the one coming from stars (dotted line), estimated from the $3.6 \mu \mathrm{m}$ flux. The observed flux densities (open rectangles) are shown along with the ones resulting when convolving the model with each band (filled circles). Although the 3.6 and $4.5 \mu \mathrm{m}$ flux densities are shown, they were not included when performing the fits, as explained in Section 4.2.

The radial trends of the different model parameters are shown in Figure 7 for each individual galaxy (only NGC 7331 is included in the printed version). In all panels, the best-fitting values are shown with different lines, and the gray bands correspond to the estimated $1 \sigma$ uncertainties. Panel (a) shows the radial variation of the $\mathrm{PAH}$ mass fraction, $q_{\mathrm{PAH}}$. Regions where $q_{\text {PAH }}$ is not reliable are identified with a dotted line instead of a solid one. In these regions, stellar emission at $8 \mu \mathrm{m}$ is more than twice the one contributed by PAHs, so even though their fitting errors might be small, they are affected by large uncertainties resulting from the stellar subtraction. Therefore, these values will not be considered in subsequent sections. The physical scale of the MIPS FWHM at $160 \mu \mathrm{m}\left(38^{\prime \prime}\right)$ at the distance of each galaxy is marked with a small horizontal line.

In panel (b), we show the fraction of dust mass exposed to high-intensity radiation fields, $\gamma$. The dotted and dashed lines correspond to the fraction of the dust-luminosity contributed by dust grains in those regions, heated by starlight with $U>U_{\text {min }}$ and $U>10^{2}$, respectively. Note the different scales for the mass and luminosity fractions: about $1 \%$ of the dust mass can be responsible for $\sim 10 \%$ of the total dust luminosity.

Panel (c) displays the radial trends of the minimum starlight intensity $U_{\min }$, as well as an average value $\langle U\rangle$, which depends on both $U_{\min }$ and $\gamma$ (see Equation (33) in D07). With this definition, we have $L_{\text {dust }} \propto\langle U\rangle M_{\text {dust }}$.

Finally, in panel (d) we show the radial profiles of both dust mass and luminosity surface densities, corrected for inclination using the semiaxis ratio in Table 1 . The open circles show the 
dust luminosity computed from the monochromatic fluxes from 8 to $160 \mu \mathrm{m}$, using Equation (22) from DL07. These values compare well with the ones obtained by integrating the SED from the model (dashed line).

\subsubsection{Properties of the Whole Sample}

In Figure 8, we show the radial variation of each parameter in the model for all galaxies in the sample, divided into bins of morphological type. The radial coordinate is normalized to the optical radius, $\mathrm{R} 25$. The median profiles in each panel are shown as a thick dashed line.

The top row shows how the PAH abundance changes with the galactocentric distance. Note that we only plot those values of $q_{\mathrm{PAH}}$ satisfying the flux criteria defined in Section 4.2; nevertheless, the PAH abundances for the earliest types should be still considered with caution. The median profiles of $q_{\mathrm{PAH}}$ in S0/a-Sbc galaxies look somewhat flat, although some individual profiles exhibit a central depletion of PAHs, relative to the abundances at larger radii. The overall $\mathrm{PAH}$ abundance in these spirals ranges from $2 \%$ to $4.6 \%$ of the total dust mass. As we move toward later types, steeper radial gradients become more frequent, and $q_{\text {PAH }}$ decreases down to $\sim 1.5 \%$ or even less for the latest types. As we will see later, this can be interpreted in terms of a connection between the PAH abundance and metallicity.

The fraction of the dust mass that is heated by very intense starlight clearly decreases with radius. This component is mostly determined by the flux density at $24 \mu \mathrm{m}$, which usually outlines the spiral pattern in which star-forming regions are typically arranged. The spatial filling factor of the $\mathrm{H}$ II regions is thus larger at the innermost regions, where they swirl and crowd together, and where sometimes they coincide with rings, whereas at larger galactocentric distances they are more dispersed, so their contribution to the $24 \mu \mathrm{m}$ surface brightness is not so important in comparison with the diffuse one. Indeed, the radial decrease in $\gamma$ is steeper in early-type grand-design spirals, while in late-type flocculent ones the gradient is flatter, since the spatial distribution of star-forming regions is more uniform. This is in agreement with the findings of Bendo et al. (2007), who also concluded that the $24 \mu \mathrm{m}$ emission is more centrally concentrated in early-type spirals than in late-type ones. In any case, although the values of $\gamma$ are quite small (typically around 1\%), such a small fraction of the total dust mass can be responsible for a non-negligible fraction of the total emitted IR power.

The scale factor $U_{\min }$ describing the intensity of the diffuse heating starlight also decreases with radius. This quantity is affected by both young and old stellar populations, and the bulge can certainly be responsible for part of that heating, raising the central values of $U_{\mathrm{min}}$, especially in Sa-Sbc spirals. Additionally, the higher central concentration of star-forming regions in these galaxies may also increase the diffuse interstellar radiation field (besides boosting $\gamma$ ). In the outer regions of spiral disks, beyond $r \sim 0.5 \mathrm{R} 25$, the median value of $U_{\min }$ is close to 1 , meaning that the radiation field has the same intensity as the local MW one. Noteworthy, in the latest types both $U_{\min }$ and $\gamma$ present higher median values, although with more dispersion.

The radial variation of the dust mass surface density also depends on the Hubble type. The largest amounts of dust are found in $\mathrm{Sb}-\mathrm{Sd}$ galaxies, where the surface density decreases exponentially from $10^{6} M_{\odot} \mathrm{kpc}^{-2}$ in the central regions to $10^{4} M_{\odot} \mathrm{kpc}^{-2}$ in the outer ones, at the $\mathrm{R} 25$ radius. Conversely, dust is less abundant in Sdm and irregulars, as well

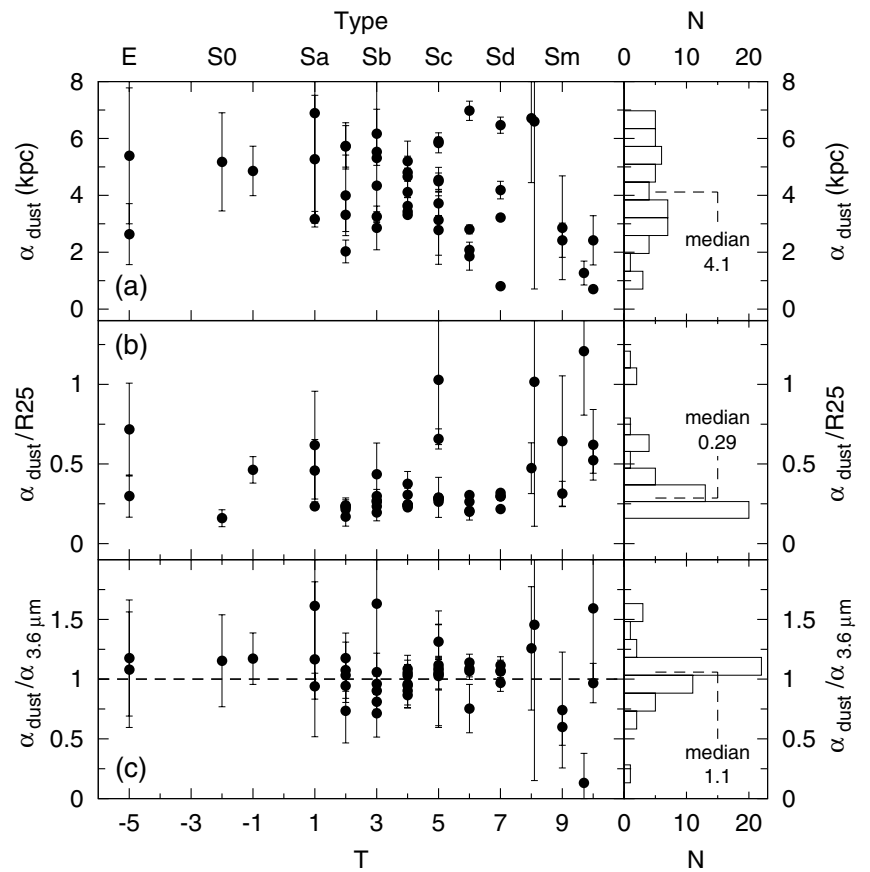

Figure 9. Radial exponential scale lengths of the dust profiles for different morphological types. The scale lengths were derived from linear fits applied to the profiles after having excluded the bulges (see the text). They are expressed in $\mathrm{kpc}$ (a), in units of the optical radius (b), and in units of the stellar scale length (c), measured on the $3.6 \mu \mathrm{m}$ profiles derived from the degraded IRAC images (bulges excluded). The global distributions for the whole sample, as well as the corresponding median values, are shown to the right.

as in ellipticals and lenticulars, with S0/a-Sab spirals lying in between.

It is worth noting that the profiles of dust mass surface density appear to be exponential, and the radial scale length seems to hold rather constant from Sab to Sd spirals, when expressed in units of the optical size. To quantify this behavior, we have applied linear fits to these profiles. We have excluded prominent bulges from the fit, in the same way as we did in Section 3.1 with the attenuation profiles. This is particularly important in many S0/a-Sab galaxies, in which the central regions usually deviate from the exponential trend, due to holes or depletions in the amount of dust (see the full version of Figure 7 in the online edition of the journal).

The resulting exponential scale lengths $\alpha_{\text {dust }}$, defined so that $\Sigma_{M_{\text {dust }}} \propto e^{-r / \alpha_{\text {dust }}}$, are shown in Figure 9 . When expressed in absolute physical units (panel a), the dust mass scale length varies widely across the Hubble sequence from 1 to $7 \mathrm{kpc}$. However, the distribution appears strongly peaked when normalizing by the optical size (panel b) and by the scale length of the stellar mass profiles (panel c), the latter obtained from the profiles measured on the degraded IRAC $3.6 \mu \mathrm{m}$ images, for consistency. The dust mass radial scale length is equal to $0.2-0.3 \mathrm{R} 25$ for Sab-Sd galaxies. The profiles are flatter in later types (although the dispersion is larger, as these systems might deviate from the disk-like geometry). In general, the dust scale length seems to be $\sim 10 \%$ larger than the stellar one. By applying a three-dimensional radiative transfer model to seven edge-on spirals, Xilouris et al. (1999) concluded that the dust radial scale length is $\sim 40 \%$ larger than the stellar one. The presence of a cold dust component $(T<15 \mathrm{~K})$ with a larger scale length could explain this difference, as this component would be detected in absorption by the radiative transfer modeling, but it would be unconstrained by our MIPS profiles. Conversely, some 


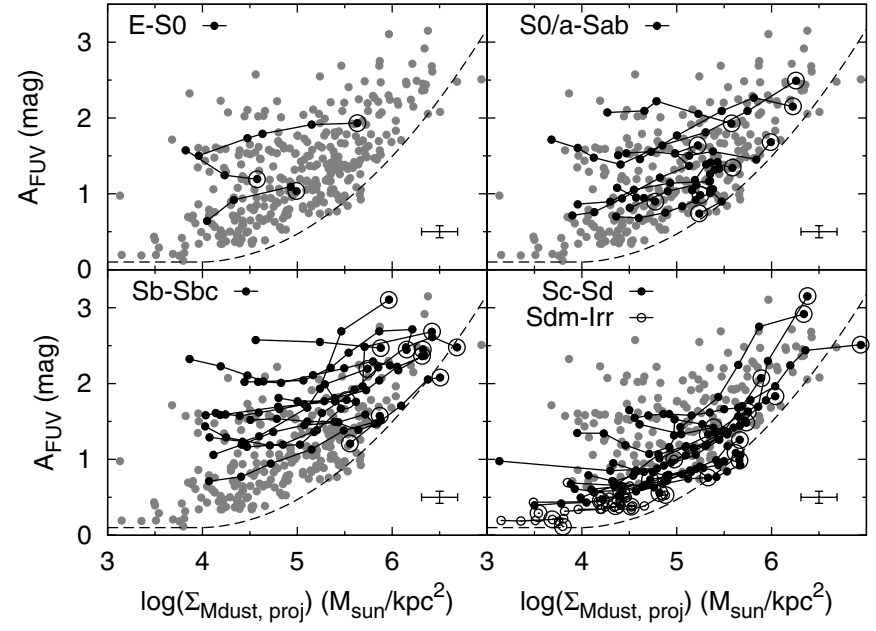

Figure 10. Attenuation in the FUV as a function of the projected dust mass surface density. Black points in each panel show the profiles of galaxies with the corresponding Hubble type. The innermost point of each profile is surrounded by a larger circle. As a reference, the data for the whole set of annular regions are shown as gray points in each of the panels. The dashed line is a fit of the lower envelope of the whole data set, performed with the boundary-fitting code presented in Cardiel (2009) (see the text for details).

of the necessary geometrical simplifications on which this kind of three-dimensional models rely could introduce some bias. For instance, the dust profiles are assumed to be exponential throughout the whole disk, which does not seem to be the case in many early-type spirals exhibiting central plateaus or holes in their dust profiles. Indeed, if we do not exclude the bulges when applying linear fits to our dust profiles, the resulting dust scale lengths are $\sim 20 \%$ larger than the stellar ones for the whole sample, and $\sim 30 \%$ larger if we focus on early-type spirals, whose bulges are more prominent.

The bottom row in Figure 8 shows the profiles of the dust light-to-mass ratio, which is essentially proportional to $\langle U\rangle$. Therefore, these profiles reflect radial changes in temperature which, in general, seems to decrease with radius. Interestingly, irregulars can reach larger dust luminosity surface densities than $\mathrm{Sa}-\mathrm{Sd}$ spirals, in spite of having considerably lower dust mass densities, thanks to their dust being hotter (note their higher values of both $U_{\min }$ and $\gamma$ ).

Note that we cannot speak of "temperature" in the same way as when fitting FIR data with a double blackbody SED, since the dust models used here consider the full temperature distribution for all grain sizes. Although the smallest grains can undergo extreme temperature changes in small periods of time, large ones dominating the FIR emission can be considered to have reasonably steady temperatures $T \approx 17 U^{1 / 6} \mathrm{~K}$ (DL07). As a sanity check, for NGC 7331 we obtain a roughly flat profile with $U_{\min } \approx 2.5$, implying $T \approx 20 \mathrm{~K}$, the same value found by Thilker et al. (2007) when applying a double blackbody fit. By analyzing the IR SEDs of different regions in M 81, Pérez-González et al. (2006) found that the temperature of the cold component varies from $\approx 22 \mathrm{~K}$ in the central regions to $\approx 15 \mathrm{~K}$ at $10 \mathrm{kpc}$ from the center, which is consistent with our $U_{\text {min }}$ profile ranging from 5 to 0.7 in the same relative radial range.

\subsubsection{Dust Mass Surface Density and Attenuation}

An initial comparison of the dust mass surface density profiles in Figure 8 and the attenuation profiles in Figure 1 shows that both quantities follow similar global trends along the Hubble

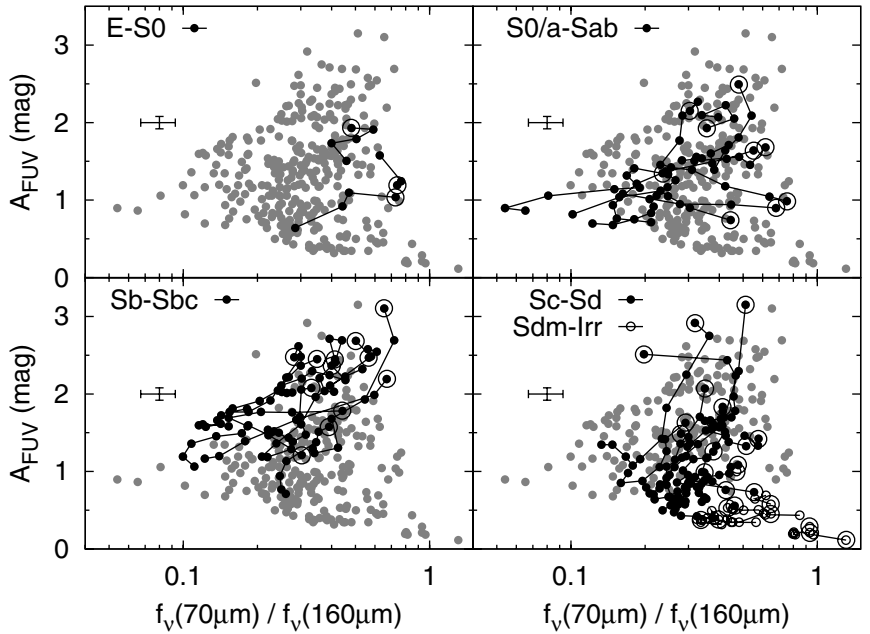

Figure 11. Attenuation in the FUV as a function of the $f_{v}(70 \mu \mathrm{m}) / f_{v}(160 \mu \mathrm{m})$ color. Black points in each panel show the profiles of galaxies with the corresponding Hubble type. The innermost point of each profile is surrounded by a larger circle. As a reference, the data for the whole set of annular regions are shown as gray points in each of the panels.

sequence, peaking at $\mathrm{Sb}-\mathrm{Sbc}$ spirals. However, the dust mass profiles show a smaller dispersion than the extinction profiles within each morphological-type bin (see also the histograms in Figures 2 and 9).

To further analyze these differences, in Figure 10 we plot the attenuation in the FUV as a function of the corresponding projected dust mass surface density (we cannot use the deprojected one, for the measured values of $A_{\mathrm{FUV}}$ take into account all the dust along the line of sight). Note that since $A_{\mathrm{FUV}}$ has been computed with the age-dependent calibration of Cortese et al. (2008), the varying relative contribution of young and old stars to the dust heating in galaxies with different SFHs is being taken into account. It could still be a problem in ellipticals and lenticulars, though.

This plot reveals that, for a given value of the dust mass density, there is always a minimum associated attenuation. This value increases monotonically from almost zero for $\Sigma_{M_{\text {dust, proj }}} \sim$ $10^{3} M_{\odot} \mathrm{kpc}^{-2}$ to roughly 3 mag for $\Sigma_{M_{\text {dust, proj }}} \gtrsim 10^{6} M_{\odot} \mathrm{kpc}^{-2}$. This lower envelope for the distribution of data points is delineated by Sc galaxies and later, while for the same amount of dust, earlier spirals present attenuations 1 or 2 mag higher. Using the boundary-fitting code described in Cardiel (2009), we obtained the following analytic parameterization for the minimum attenuation at a given dust column density:

$$
A_{\mathrm{FUV}, \min } \approx \begin{cases}5.16-2.57 x+0.327 x^{2} & \text { if } 4<x<7 \\ 0.1 & \text { if } 3<x<4\end{cases}
$$

where $x=\log \Sigma_{M_{\text {dust, proj }}}$, in $M_{\odot} \mathrm{kpc}^{-2}$. This fit is shown in Figure 10 with a dashed line.

The scatter in $A_{\mathrm{FUV}}$ found for each given $\Sigma_{M_{\text {dust, proj }}}$ can be attributed to the relative distribution of stars and dust within each galaxy. It is known that for geometries more complex than a foreground homogeneous screen of dust, the distribution of dust grains relative to the heating sources overcomes the net amount of dust as the main factor determining the attenuation (Calzetti 2001).

To illustrate this issue, in Figure 11 we plot $A_{\mathrm{FUV}}$ as a function of the far-IR color. Data points are clearly arranged in a wedge-like distribution, completely analogous to the one 
found by Dale et al. (2007) using the integrated photometry of the SINGS galaxies. These authors came to the conclusion that the location of galaxies in this diagram was closely related with their morphology in the $24 \mu \mathrm{m}$ band. Galaxies with clumpy morphologies were found to have warm far-IR colors but low attenuations: while there are significant amounts of hot dust concentrated around heating sources in star-forming regions, many UV photons can find their way out of the galaxy through lines of sight clean of dust, thus decreasing the overall TIR-toFUV ratio. In our radial analysis, Sc spirals and later clearly lie in the lower right corner of the plot, thus being consistent with having a clumpy distribution of dust relative to stars. The presence of these kind of porous star-forming regions has been identified in nearby galaxies (Roussel et al. 2005), and their contribution to the TIR-to-FUV ratio may become progressively dominant with increasing galactocentric distance.

In contrast, Dale et al. (2007) found that the upper right region of the plot is populated by galaxies showing centrally concentrated emission at $24 \mu \mathrm{m}$. In our radially resolved analysis the central regions of spirals, especially in Sb-Sbc ones, lie in this area of the diagram. This indicates that UV light has significant difficulties to avoid dust, thus leading to much larger extinction values. Finally, regions having cooler far-IR colors tend to exhibit intermediate attenuations. This area of the plot was identified by Dale et al. (2007) as corresponding to galaxies with a smooth $24 \mu \mathrm{m}$ morphology. This is also the case for our radial profiles, since the disks of Sbc spirals and earlier populate this zone of the plot. While not being as heavily extincted as in the central regions, UV photons in these disks still find it difficult to leak through dust, in comparison with clumpy Sc-Sd spirals, in which the attenuation is lower despite having similar dust mass surface densities.

\subsubsection{PAH Abundance}

Over the years, a body of observational evidence has been collected showing that PAH emission is weak or even suppressed in low-metallicity systems (see, e.g., Boselli et al. 1998; Sturm et al. 2000; Madden 2000; Madden et al. 2006; Hunt et al. 2005; Hogg et al. 2005; D07; Engelbracht et al. 2005, 2008). However, the physical mechanisms driving the observed correlation between PAH abundance and metallicity remain a subject of investigation.

Several authors have invoked an evolutionary origin for this trend (see, e.g., Galliano et al. 2008; Dwek et al. 2009). PAHs are thought to form in the envelopes around carbon-rich AGB stars, being later injected into the ISM through a recycling process that takes place over timescales of a few Gyr. The remaining dust species are believed to condense out of ejecta from $\mathrm{SNe}$ in much shorter timescales. Thus, most of the dust in young, low-metallicity systems would be made out of SNcondensed species, whereas PAH particles would still require more time to form. Hence, the different speed of both processes would naturally lead to a progressive increase in $q_{\text {PAH }}$ with time, explaining the observed PAH-metallicity trend. This scenario, however, should be considered cautiously, as many aspects of chemical evolution and dust formation still remain unclear. For instance, despite their low metallicities, some dwarf galaxies may harbor underlying stellar populations a few Gyr old. Also, carbon dust might be also produced in the ejecta of $\mathrm{SNe}$, and its abundance relative to carbon dust due to AGB stars should also be taken into account.

Conversely, other possible explanations rely on destructive mechanisms of PAH particles. Systems with younger stellar

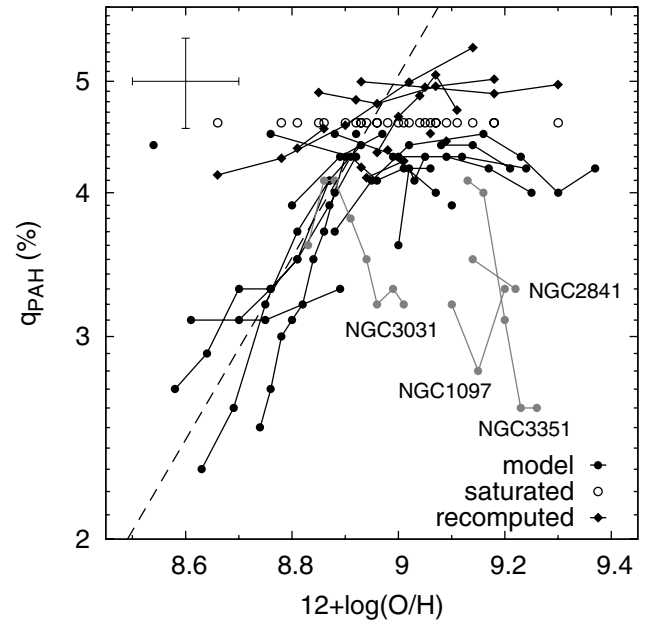

Figure 12. Fraction of the dust mass contributed by PAHs against the metallicity at each radial bin. Data points with $q_{\mathrm{PAH}}=4.6 \%$ are "saturated," meaning that their PAH abundances correspond to the current upper limit of the models (see Section 4.3.4). For these points, we have recomputed $q_{\text {PAH }}$ from the empirical fit presented in Appendix A.2 (see also Figure 18). The dashed line is a bisector linear fit applied to the data points below $12+\log (\mathrm{O} / \mathrm{H})=9$, and corresponds to $q_{\mathrm{PAH}} \propto(\mathrm{O} / \mathrm{H})^{0.8 \pm 0.3}$. Four galaxies for which the $q_{\mathrm{PAH}}$ vs. $(\mathrm{O} / \mathrm{H})$ trend is reversed have been highlighted (see the text for details).

populations have harder UV radiation fields, given their larger fraction of ionizing OB stars. Furthermore, PAHs are likely to be less shielded by dust from these hard photons than in more metal-rich systems, since low-metallicity galaxies usually exhibit lower dust attenuation. There is observational evidence showing a progressive paucity of aromatic features as the hardness of the radiation field increases (Engelbracht et al. 2005, 2008; Madden et al. 2006; Bendo et al. 2006, 2008; Smith et al. 2007; Gordon et al. 2008).

It is not straightforward to disentangle the relative contribution of formation and destruction effects to the observed PAH abundances. As we commented above, predominantly young systems which are expected to show a paucity of PAHs because of chemical evolution, also exhibit hard radiation fields that could destroy the already present ones. Besides, variations in the aromatic emission could be due to changes in the ionization state of PAHs rather than to a real decrease in terms of abundance. Wu et al. (2006) found that the EW of the aromatic features correlates better with a parameter simultaneously taking into account both the starlight hardness and the metallicity than with any of those parameters alone.

To check whether the PAH-metallicity trend holds true in our radial analysis, in Figure 12 we plot the PAH abundance at each galactocentric distance as a function of the corresponding metallicity at the same radial bin, for the 22 galaxies in common with the sample of J. Moustakas et al. (2009, in preparation). As in Section 3.3, we only trust those metallicities interpolated within the spatial range covered by $\mathrm{H}$ II regions in the aforementioned study. As explained in Section 3.3, we adopt the metallicity values based on the Kobulnicky \& Kewley (2004) calibration. Data points falling outside that range require uncertain extrapolations of the metallicity gradients, and have been excluded from this analysis, as well as regions with excessive stellar emission at $8 \mu \mathrm{m}$ (see Section 4.2).

There is a general trend of $q_{\mathrm{PAH}}$ to increase with the oxygen abundance for $12+\log (\mathrm{O} / \mathrm{H})<9$. Note that in Sdm and irregular galaxies, the PAH abundance ranges from $2.5 \%$ to less than $1 \%$. However, there are not enough individual measurements 

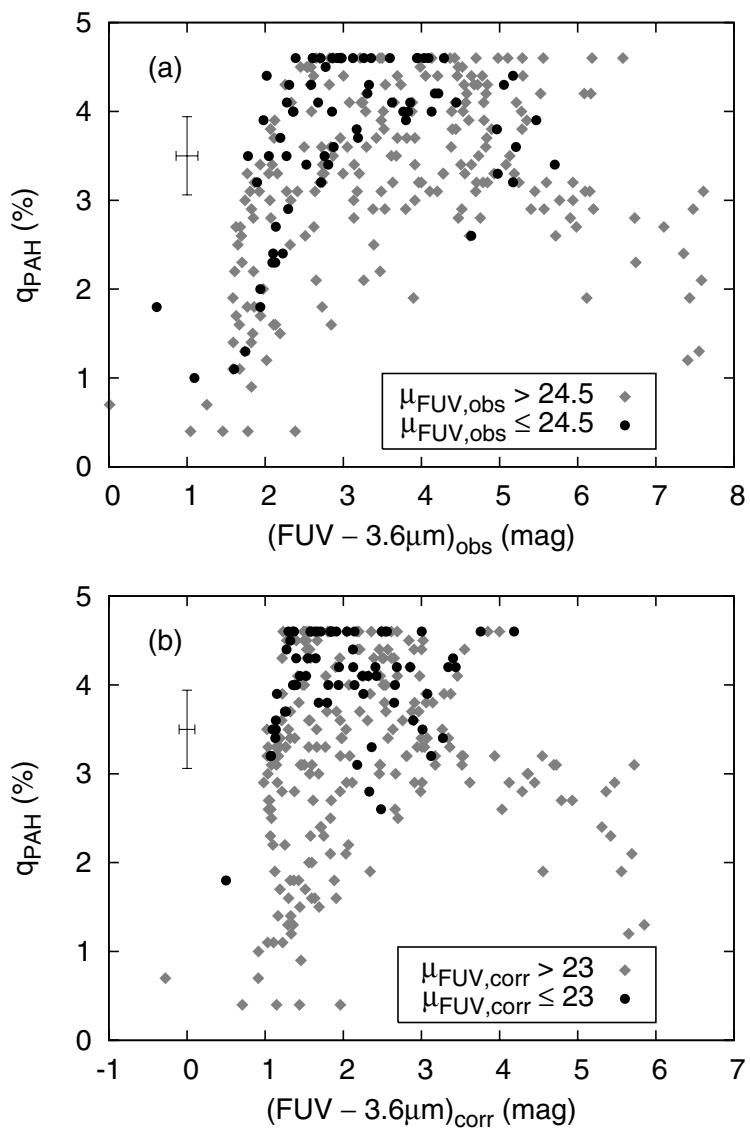

Figure 13. PAH abundance against the observed (a) and extinction-corrected (b) (FUV-3.6 $\mu \mathrm{m}$ ) color. Data points are sorted out depending on their surface brightness in the FUV band (in mag arcsec ${ }^{-2}$ ). To allow for a fair comparison between both panels, an offset of $1.5 \mathrm{mag}$ has been applied to the surface brightness value chosen to classify the data points, since that is the typical FUV attenuation found in our sample.

of $\mathrm{H}$ II regions in these galaxies to derive metallicity gradients, which precludes making use of those data points for this plot. The available abundance data indicate that these galaxies have $12+\log (\mathrm{O} / \mathrm{H})<8.6$ (J. Moustakas et al. 2009, in preparation), so the monotonic trend between $q_{\mathrm{PAH}}$ and $(\mathrm{O} / \mathrm{H})$ continues down to lower abundances. This general trend is consistent with that found by D07 in the SINGS galaxies using galaxyaveraged PAH abundances and metallicities. The dashed line, which corresponds to a bisector linear fit applied to the data with $12+\log (\mathrm{O} / \mathrm{H})<9$, shows that $q_{\text {PAH }} \propto(\mathrm{O} / \mathrm{H})^{0.8 \pm 0.3}$ in this range of metallicities. The slope may vary within individual galaxies, and it could be different for lower oxygen abundances. We call attention to the fact that, up to now, integrated photometry usually required comparing normal spirals with dwarf galaxies in order to detect this trend, while radial profiles make it possible to see it within a more limited range of galaxy types because of the reduced scatter.

At larger metallicities, however, the slope flattens and even reverses. In principle, the upper limit of the DL07 models for the PAH abundance $(4.6 \%)$ could be the culprit, but we call attention to the fact that in most cases this flattening arises before that limit is reached, and it remains visible when recomputing the PAH abundances with the empirical recipes presented in Appendix A.2. Interestingly, some models describing the stellar evolutionary effects on the chemical composition of dust predict that such a change in slope might actually occur (Galliano et al. 2008), and attribute it to the different carbon content of AGB stars with different masses - and hence with different lifetimes. Stars with masses between 1.2 and $3.0 M_{\odot}$ are thought to end up as carbon-rich post-AGB stars, unlike low-mass stars, which remain oxygen-rich (see, e.g., García-Lario (2006) and references therein). Since the latter have longer lifetimes, they contribute later to the enrichment of the ISM. Moreover, the C/O ratio in AGB stars also seems to depend on the stellar metallicity, with more metal-rich AGBs yielding lower amounts of carbon (see Dwek et al. (2009) and references therein). As a result, these models predict that the formation of carbon dust from AGB stars slows down with time, while the absolute abundance of SN-condensed dust species keeps rising.

There are four galaxies in Figure 12 that deserve special attention, for they have low PAH abundances compared to other galaxies with similar metallicities; moreover, the trend between those quantities is actually reversed within each one of these objects. Interestingly, they are the earliest spirals in the diagram (Sb and earlier). Therefore, they have presumably undergone rapidly declining SFHs, with typical timescales of just a few Gyr (Gavazzi et al. 2002), in contrast to late-type spirals, which exhibit longer timescales. If SFH is driving the progressive change of slope due to the different carbon content of AGBs with different masses and/or metallicities, this effect could be enhanced in systems with rapidly declining SFHs.

In Figure 13, we show how the PAH abundance changes with the observed and extinction-corrected (FUV-3.6 $\mu \mathrm{m}$ ) color. The color of the radiation field illuminating the PAHs probably lies between the observed and extinction-corrected values because it may be partially reddened by dust. Regions with the reddest colors, usually the bulges of early-type spirals, exhibit low or intermediate PAH abundances, as we already saw in Figure 8. But for (FUV-3.6 $\mu \mathrm{m}$ ) corr colors between 0 and 4 mag (the range in which star-forming disks are typically found, as shown in Figure 3), PAHs progressively become less abundant in the outer and bluer regions, although the trend is noisy and mainly defined by a lower envelope. If photodestruction of PAH particles by intense UV fields overcame PAH formation by AGB stars, it would be conceivable to observe systematic differences in $q_{\mathrm{PAH}}$ by sorting the data points according to their FUV surface brightness ( $\left.\mu_{\mathrm{FUV}}\right)$ for a fixed (FUV-3.6 $\mu \mathrm{m}$ ) color, despite its limitations (see below). UV bright regions should populate the lower left area of Figure 13 if PAHs are being selectively destroyed in them, but they seem to have comparable PAH abundances as the remaining regions, since these brightest zones are usually the central, more evolved ones.

Since we are lacking spatially resolved information from ionization tracers (such as the $[\mathrm{Ne} \mathrm{III}] /[\mathrm{Ne}$ II $]$ ratio), we are relying on the FUV surface brightness as a rough proxy for the level of ionization. This choice would be more justified in a pixel-to-pixel analysis, since $\mu_{\mathrm{FUV}}$ would then allow us to identify regions of massive star formation, but this analysis is beyond the scope of this paper. However, the usefulness of $\mu_{\mathrm{FUV}}$ in radial profiles as a ionization tracer is much more limited. It tends to select the central and brightest zones of the disks, where the evolutionary origin reflected by metallicity seems to be more important. In the outer regions, however, the azimuthally averaged FUV surface brightness is fainter, even if localized massive star formation is present. In other words, the declining FUV profiles do not imply that the UV field within individual star-forming regions becomes softer as we move away from the center of the galaxy. Indeed, it is worth noting that in M 33 the hardness of the UV radiation field inside single $\mathrm{H}$ II regions is seen to increase with galactocentric distance 
(Rubin et al. 2008). Since our PAH abundances are not limited to individual $\mathrm{H}$ II regions, but take into account all the aromatic emission within elliptical annuli, the corresponding ionization tracer should reflect the average radiation field heating all that dust, which is likely to be softer than the one present in the core of star-forming regions. With the available data, $\mu_{\mathrm{FUV}}$ is possibly our best choice, but in any case the results concerning the relative importance of PAH destruction are not conclusive and should be treated with caution.

The whole analysis presented in this section supports the idea that, at least in normal spiral galaxies and in spatial scales larger than those of single $\mathrm{H}$ in regions, the observed trends between the PAH mass fraction and metallicity or (FUV-3.6 $\mu \mathrm{m}$ ) color may have an evolutionary origin. We emphasize, however, that while the varied timescales in which different elements are injected in the ISM may be partly responsible of the observed dependence of the $\mathrm{PAH}$ mass fraction on $\mathrm{O} / \mathrm{H}$, other processes governing the balance of dust formation and destruction may be correlated with metallicity as well.

In their analysis of individual $H$ II regions in $M 101$, Gordon et al. (2008) find that the EW of the aromatic features is better correlated with the level of ionization than with metallicity, and this seems to hold for the starburst galaxies studied by Engelbracht et al. (2008). Rather than representing a contradiction with other studies (including ours), these results reflect diverse behaviors associated with the different regimes of physical conditions being probed. While photodestruction of PAH particles by hard UV photons may overcome evolutionary effects in the core of $\mathrm{H}$ II regions and in starburst galaxies, it does not seem to play such an important role at larger spatial scales in our galaxies. Indeed, Gordon et al. (2008) note that this trend with the hardness of the radiation field only appears above a certain level of ionization. Below this level the trend seems to vanish, implying that PAHs are not being destroyed faster than the remaining dust species. This is likely the case in our azimuthally averaged profiles, where we are measuring aromatic features emerging not only from the very inside of $\mathrm{H}$ II regions, but also from the diffuse ISM, where PAHs are not necessarily exposed to such levels of ionization. From the SED decomposition shown in Figure 6 we can see that, in general, the dust emission at 5.8 and $8.0 \mu \mathrm{m}$ is dominated by the diffuse component. Therefore, at these spatial scales chemical evolution seems to act as the main driver of the observed trends, rather than selective PAH destruction. Recent analysis of the dust emission in NGC 0300 (Helou et al. 2004), NGC 4631 (Bendo et al. 2006) and a subset of 15 SINGS spirals (Bendo et al. 2008) seem to further support this general picture. As in Gordon et al. (2008), these authors found that the PAH emission at $8 \mu \mathrm{m}$ and the hot dust radiation at $24 \mu \mathrm{m}$ are correlated in scales of a few kpc, but this correlation breaks down within individual star-forming regions, where the PAH emission is less centrally peaked than the one contributed by very hot dust.

\subsubsection{Photometric $8 \mu \mathrm{m}$ Equivalent Width}

Another important issue to address is how the PAH abundance-as a fraction of the total dust mass-relates to the EW of the observed spectral aromatic features. Although two-dimensional IRS spectral maps are available for most of the SINGS galaxies, they do not usually cover the full spatial extent of these objects. In this regard, Engelbracht et al. (2008) propose a photometric method to estimate the EW of the $7.7 \mu \mathrm{m}$ feature using the stellar-subtracted flux at $8.0 \mu \mathrm{m}$

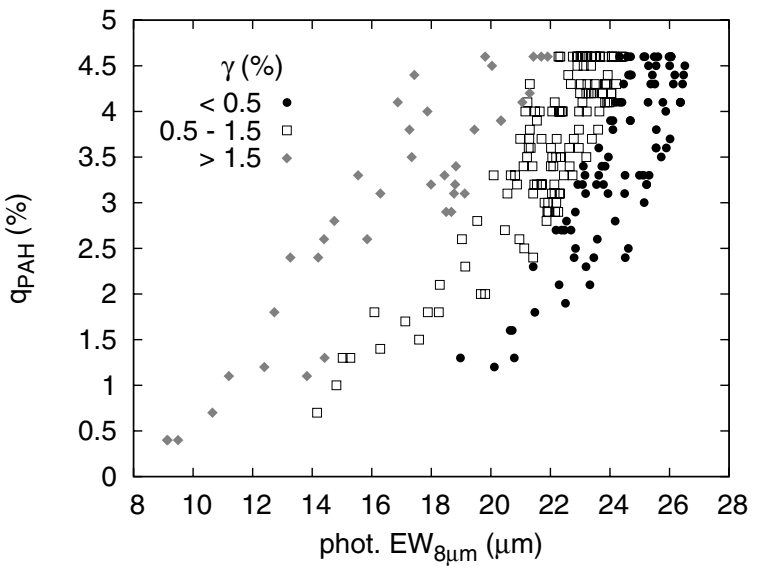

Figure 14. PAH abundance against the photometric estimator of the $8 \mu \mathrm{m} \mathrm{EW}$, computed following the prescriptions of Engelbracht et al. (2008). Different symbols correspond to different fractions of the total dust mass heated by very intense starlight.

band, and a logarithmic interpolation of the stellar-subtracted fluxes at 4.5 and $24 \mu \mathrm{m}$ to estimate the underlying continuum. Note that, as pointed out by these authors, the $8 \mu \mathrm{m}$ EW (either the spectroscopic or its photometric proxy) is a measurement of the abundance of PAHs relative to small grains or very hot larger ones, which contribute to the underlying continuum at those wavelengths. Translating the $8 \mu \mathrm{m} \mathrm{EW}$ into an abundance relative to the total dust mass can be challenging without a prior knowledge of the temperature distribution of the dust grains, as we will see below.

It is not easy to obtain this photometric estimator for the $8 \mu \mathrm{m}$ EW directly from our data. Although recovering the dustonly emission at 8.0 and $24 \mu \mathrm{m}$ by means of the stellar factors presented in Section 4.2 does not pose any problem, computing the non-stellar flux at $4.5 \mu \mathrm{m}$ is not straightforward, since the emission at that band is almost entirely dominated by stars (the sample studied by Engelbracht et al. (2008) consists of starburst galaxies usually exhibiting less stellar contamination at that band). Indeed, in our sample the stellar emission at $4.5 \mu \mathrm{m}$, extrapolated from the flux at $3.6 \mu \mathrm{m}$, is sometimes slightly larger than the actual observed values (partly due to photometric uncertainties, and to the fact that these stellar factors are average values). Alternatively, here we use the nonstellar fluxes corresponding to the best-fitting model for each galactocentric distance, instead of recovering them from the observed data.

In Figure 14, we plot the PAH abundance derived from the models against the photometric EW estimated following the prescriptions given by Engelbracht et al. (2008). Both quantities are positively correlated, but there is a clear dependence on the fraction of dust exposed to intense starlight: at fixed $q_{\mathrm{PAH}}$, the photometric proxy for the EW decreases as the fraction $\gamma$ of hot dust increases. This is most likely due to the effect that $\gamma$ has on the flux at $24 \mu \mathrm{m}$ : as seen in previous sections, exposing a small amount of dust to very intense radiation fields can significantly increase the observed emission at that band, and thus increase the interpolated continuum at $8 \mu \mathrm{m}$. Therefore, the resulting EW will result in smaller values. In conclusion, the $8 \mu \mathrm{m}$ EW may be used to compare the PAH mass fraction in samples of galaxies not presenting large variations in their FIR SEDs, but should be used with caution if this is not the case. 


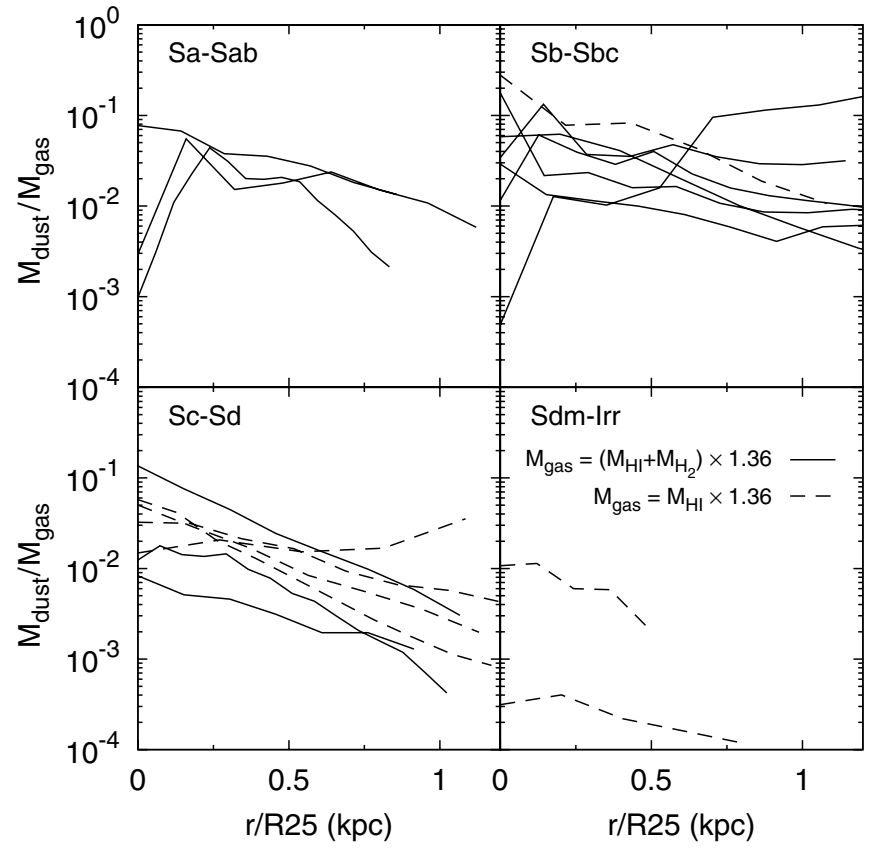

Figure 15. Radial variation of the dust-to-gas ratio for the SINGS-THINGS galaxies, divided into different bins of morphological types. The radial axis is normalized to the optical size of each galaxy, as given by the R25 radius. Galaxies with available $\mathrm{H}$ I and $\mathrm{CO}$ profiles are shown with solid lines, whereas those lacking CO data appear as dashed lines (their profiles are then upper limits, although most of the molecular gas is expected to be located in the innermost regions). In all but two galaxies, a metallicity-dependent $\mathrm{CO}-$ to- $\mathrm{H}_{2}$ conversion factor changing with radius was used (see the text). In all plots throughout this paper explicitly involving $(\mathrm{O} / \mathrm{H})$, we only trust metallicity values within a restricted spatial range defined by the $\mathrm{H}$ II regions used to determine the metallicity gradients. In this figure, however, the accuracy of the oxygen abundance is less critical, since it constitutes a second-order effect in comparison with the assumption of a constant $\mathrm{CO}-$ to- $\mathrm{H}_{2}$ factor. Therefore, in this particular plot we have not applied these restrictions to the metallicity values when computing the $\mathrm{H}_{2}$ mass surface densities.

\subsubsection{Dust-to-gas Ratio}

The dust-to-gas ratio is an important quantity when studying the chemical enrichment of the ISM, since it tells us about the amount of metals that get locked up in the dust through the stellar yields. Indeed, a correlation has been widely shown between the dust-to-gas ratio and the gas-phase oxygen abundance (e.g., Lisenfeld \& Ferrara 1998; Edmunds 2001; James et al. 2002; Hirashita et al. 2002; D07). In their analysis of a small sample of eight nearby galaxies (including the MW), Issa et al. (1990) concluded that the dust-to-gas ratio (as traced by the $A_{V} / N_{H}$ ratio) decreases with radius, following a trend with metallicity. A similar result was found by Boissier et al. (2004), who confirmed that a similar trend arises when the dust mass is directly estimated from the FIR emission, rather than from the attenuation.

In order to obtain total gas profiles, we combined $\mathrm{H}$ I data from the THINGS survey with CO profiles compiled from the literature (see Table 1), which were transformed into $\mathrm{H}_{2}$ profiles by means of the metallicity-dependent CO-to- $\mathrm{H}_{2}$ conversion factor of Boselli et al. (2002). While this approach certainly constitutes an improvement over using a constant conversion factor, the derived $\mathrm{H}_{2}$ profiles should be still treated cautiously. The CO-to- $\mathrm{H}_{2}$ conversion factor may depend on parameters such as the cloud density and the excitation temperature of the $\mathrm{CO}$, both of which could potentially vary systematically with galactocentric radius. Also, note that whereas the $\mathrm{H} \mathrm{I}$ profiles were obtained in a consistent way with respect to the UV and IR data by measuring on the $\mathrm{H}$ I intensity maps degraded to the $160 \mu \mathrm{m}$ resolution, the CO profiles were directly interpolated at different radii from published profiles. Hence, the $\mathrm{CO}$ profiles might not necessarily match exactly the same spatial regions. Note also that since no metallicity gradients are available for NGC 4826 (Sab) and NGC 3627 (Sb), we used the CO-to- $\mathrm{H}_{2}$ conversion factor corresponding to the global $\mathrm{H}$-band luminosity of each object (Boselli et al. 2002).

In Figure 15, we show the radial dust-to-gas profiles for the SINGS-THINGS galaxies, sorted by morphological type. Those galaxies for which only $\mathrm{H}$ I data are available are plotted with a dashed line, and their dust-to-gas ratios are therefore just upper limits, at least in the innermost regions where molecular gas is more likely to be found.

In most objects, the dust-to-gas ratio decreases with radius. In the central regions of $\mathrm{Sb}-\mathrm{Sd}$ spirals, it ranges between $10^{-1}$ and $10^{-2} M_{\odot} \mathrm{kpc}^{-2}$, and it decreases faster in Sc-Sd ones, reaching values close to $10^{-3} M_{\odot} \mathrm{kpc}^{-2}$ or lower at the optical radius. NGC 3627 constitutes a notable exception, for its dust-to-gas ratio increases with radius. It is an interacting $\mathrm{Sb}$ spiral in the Leo triplet that gets redder in the outer regions, possibly because of SF quenching as a result of gas removal or exhaustion. NGC 2976 is a Sc galaxy whose dust-to-gas ratio remains roughly constant across the entire optical size. This quite unusual object exhibits two bright knots of intense star formation located at almost symmetrical positions with respect to the center of the galaxy.

In Figure 16(a), we plot the dust-to-gas ratio against the gas-phase oxygen abundance, for all the SINGS galaxies with available spatially resolved metallicities, $\mathrm{H}$ I, and $\mathrm{CO}$ data. Points belonging to the same object have been connected for clarity. If we were to assume that the abundances of all heavy elements were proportional to the oxygen abundance and that all heavy elements condensed to form dust in the same way as in the MW, then the dust-to-gas ratio should scale proportionally to the oxygen abundance (D07)

$$
\frac{M_{\text {dust }}}{M_{\text {gas }}} \approx \frac{0.01}{1.36} \frac{(\mathrm{O} / \mathrm{H})}{(\mathrm{O} / \mathrm{H})_{\mathrm{MW}}},
$$

where 0.01 is the dust-to-hydrogen ratio of the MW and the factor 1.36 accounts for helium and heavier elements. The dashed line in Figure 16(a) marks this relation and shows that, at least to first order and for $12+\log (\mathrm{O} / \mathrm{H})>8.9$, the radial correlation between the dust-to-gas ratio and the metallicity follows this simple scaling law within a factor of $\sim 2$. In Figure 16(b), we plot the galaxies for which only $\mathrm{H}$ I data are available. Their values of $M_{\text {dust }} / M_{\text {gas }}$ might be overestimated if significant amounts of molecular gas are present (which is likely the case in the central regions).

We call attention to the fact that at low oxygen abundances the observed trend in panel (a) seems to be steeper than the one predicted by Equation (6). A linear fit to the whole data set yields

$$
\log \left(M_{\text {dust }} / M_{\text {gas }}\right)=5.63+2.45 \times \log (\mathrm{O} / \mathrm{H}) .
$$

In their analysis of the integrated photometry of the SINGS galaxies, D07 found that galaxies with and without available submillimeter data followed slightly different trends in the $M_{\text {dust }} / M_{\text {gas }}$ versus metallicity plots, with the slope being a bit steeper when the dust masses had been obtained without submillimeter data. 


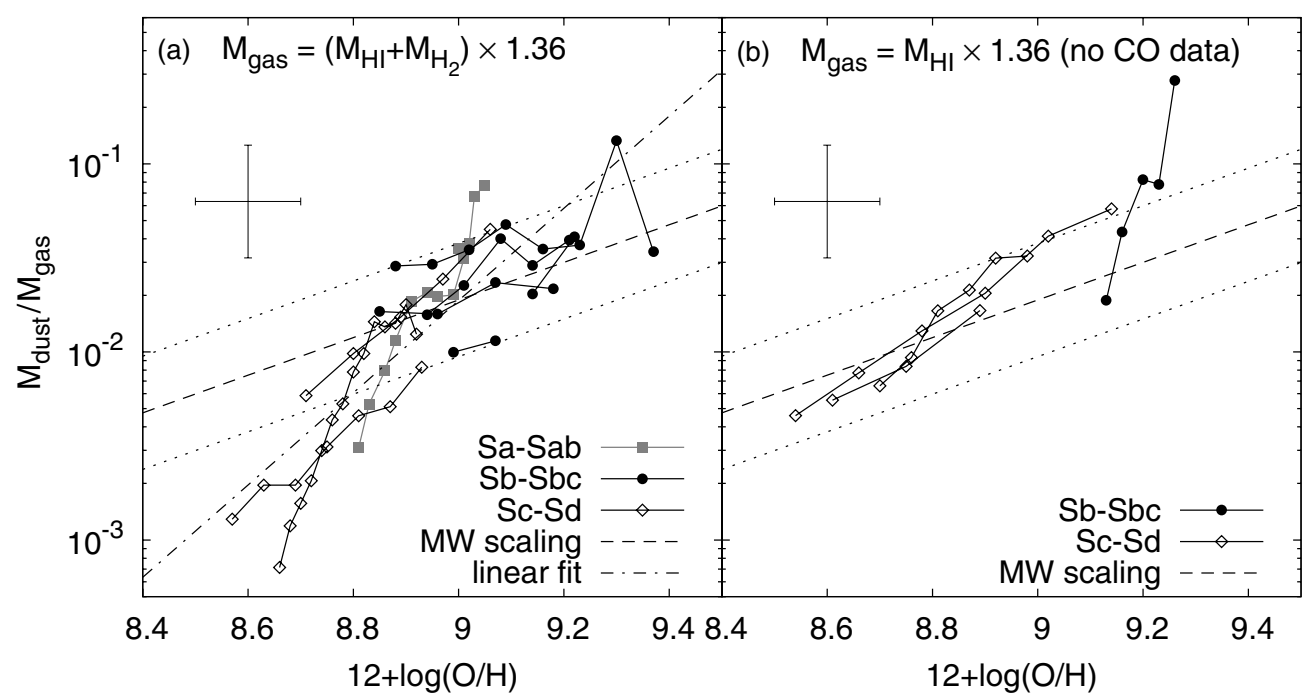

Figure 16. Dust-to-gas ratio for the SINGS-THINGS galaxies against the metallicity at different galactocentric distances. Data points belonging to the same galaxy have been connected. Different symbol shapes correspond to different morphological types. The dashed line marks the simple scaling law described by Equation (6), whereas the dotted lines show variations of a factor of 2 around it. The dot-dashed line results from a linear fit to the data. (a) Galaxies for which both $\mathrm{H}$ I and CO data are available. (b) Objects lacking $\mathrm{CO}$ data. The values at the innermost (i.e., more metallic) regions are upper limits.

It is not clear, therefore, if the apparent departure from the MW scaling law seen in Figure 16 at the lowest metallicities is real, or due to our cool dust masses being underestimated (the metallicity gradients might be also uncertain). Should this trend be real, it could link the behavior of the outermost, lower metalabundant regions of spirals with the properties of dwarf galaxies, which exhibit lower dust-to-gas ratios than those predicted by extrapolating Equation (6). Detailed models describing dust evolution in dwarf galaxies (see Edmunds 2001; Hirashita et al. 2002) usually invoke several mechanisms to either delay dust injection in the ISM or to eliminate it, via outflows caused by collective SN-driven winds. However, the extremely low dustto-gas ratios of many dwarf galaxies could be partly due to their extended $\mathrm{H}$ I envelopes, where no dust emission is apparently seen (see, e.g., Walter et al. 2007). Indeed, when $M_{\text {dust }} / M_{\text {gas }}$ is computed inside regions detected both in FIR and $\mathrm{H}$, the values derived are more consistent with Equation (6) (D07).

The extent to which these explanations proposed for dwarf galaxies are also valid for the outer regions of spirals remains unclear. The radial decrease of the star formation efficiency found in nearby spirals (Thilker et al. 2007; Leroy et al. 2008) could also partly explain the low dust-to-gas ratios seen in Figure 16. The presence of large reservoirs of gas which has not been yet transformed into stars (and hence into dust) would drop the dust-to-gas ratio in the outer regions of these galaxies.

\section{SUMMARY AND CONCLUSIONS}

We have measured multi-wavelength surface brightness profiles for the galaxies included in the SINGS sample, using UV, IR, and H I data from GALEX, Spitzer (from SINGS), and VLA (from the THINGS survey), complemented with published CO data and metallicity gradients. The analysis of this panchromatic, spatially resolved data set reveals important results regarding the dust properties of nearby galaxies.

1. We have derived radial extinction profiles by means of an SFH-dependent calibration of the TIR-to-FUV ratio. In most galaxies, the attenuation decreases with the galactocentric distance. It is largest in $\mathrm{Sb}-\mathrm{Sbc}$ galaxies, ranging from $A_{\mathrm{FUV}} \sim 2.5 \mathrm{mag}$ in the central regions to $\sim 1.5 \mathrm{mag}$ at the optical radius R25. As we move toward earlier or later Hubble types, the attenuation decreases and its radial variation becomes less pronounced. S0/a-Sab spirals typically have $A_{\mathrm{FUV}} \sim 1.5 \mathrm{mag}$, and in Sdm and irregular galaxies the extinction drops below 0.5 mag. However, the dispersion is rather large ( 1-2 mag) within each bin of morphological types. This is the result of the different spatial distribution of stars and dust (see below).

2. The TIR-to-FUV excess is correlated with the UV spectral slope $\beta$, following an IRX- $\beta$ relation shifted with respect to the one found in starburst galaxies. The main driver of this departure seems to be the SFH, with more quiescent regions having redder UV slopes for a given TIR-to-FUV ratio. Indeed, the intrinsic (FUV-3.6 $\mu \mathrm{m}$ ) color-a metric tracer of the current-to-past SFR-correlates well with the distance from the relation for starbursts. Our radial analysis reveals that this shift is particularly evident in bulges, as well as in the outer regions of anemic spirals with clear signs of star formation quenching. However, second-order factors might also play a role, since the dependence on the SFH gets completely blurred unless a wide range of SFHs is explored. We also provide a calibration to estimate the UV attenuation from the UV slope when FIR data are unavailable, which is statistically valid for "normal" starforming galaxies.

3. By combining our data with oxygen abundance gradients, we have analyzed the influence of metallicity on attenuation. Both quantities are clearly correlated, with metalricher regions exhibiting larger attenuations. Inclination plays an important role here; at fixed metallicity, the extinction is larger in galaxies that are closer to edge-on. The same happens with the UV color: more inclined galaxies appear redder than nearly face-on ones. Both effects are roughly balanced when combining both quantities in the IRX $-\beta$ plot, so that data points are just shifted along the IRX $-\beta$ relationship.

4. We have fit the FIR profiles with the dust models of Draine \& Li (2007), deriving the radial distribution of the PAH abundance, the dust mass and luminosity surface densities, and the properties of the heating starlight. The 
fraction of the dust mass in the form of PAHs $\left(q_{\mathrm{PAH}}\right)$ varies widely along the Hubble sequence, reaching a maximum of $\sim 3 \%-4.5 \%$ in $\mathrm{Sb}-\mathrm{Sbc}$ spirals, and almost disappearing $(\lesssim 1 \%)$ in the outer regions of $\mathrm{Sdm}$ and irregular galaxies. Indeed, the PAH abundance typically decreases with radius in Sc spirals and later; in earlier types, however, the profiles tend to flatten and even reverse. The PAH abundance increases with metallicity for $8.5<12+\log (\mathrm{O} / \mathrm{H})<$ 9 , and the trend progressively flattens and eventually reverses at larger oxygen abundance. This is in qualitative agreement with the predictions of models coupling stellar evolution with dust formation. Although this dependence on metallicity has already been found when comparing normal spirals with dwarf galaxies, using radial profiles leads to a considerable reduction of the dispersion and carrying out the study in a narrower range in metallicity, without the need to include low-metallicity dwarfs.

5. The average intensity of the heating starlight decreases monotonically with the galactocentric distance, and in the outer regions it usually reaches similar values as the local MW radiation field, corresponding to cold dust temperatures of $\sim 20 \mathrm{~K}$. In order to explain the observed $24 \mu \mathrm{m}$ flux densities, it is required that about $1 \%$ of the total dust mass must be exposed to very intense radiation fields. Such a small amount of dust can account for $\sim 10 \%$ of the total dust IR luminosity.

6. The dust mass surface density is largest in Sb-Sbc spirals, varying from $10^{6}$ to $10^{4} M_{\odot} \mathrm{kpc}^{-2}$ from the center to the $\mathrm{R} 25$ radius. Dust is less abundant in earlier spirals, typically having surface densities below $10^{5} M_{\odot} \mathrm{kpc}^{-2}$, and also in $\mathrm{Sdm}$ and later, where it drops below $10^{4} M_{\odot} \mathrm{kpc}^{-2}$. The dust profiles are exponential, and have a radial scale length that holds remarkably constant $(0.2-0.3 \mathrm{R} 25)$ from $\mathrm{Sb}$ to $\mathrm{Sd}$ galaxies. The dust profiles in $\mathrm{S} 0 / \mathrm{a}$ and Sab spirals usually present a central depletion, but have similar radial scale lengths than $\mathrm{Sb}-\mathrm{Sd}$ ones in the outer exponential regions. Compared to the radial distribution of old stars, as traced by the $3.6 \mu \mathrm{m}$ profiles, the dust radial scale length is, on average, $\sim 10 \%$ larger than the stellar one.

7. The relative spatial distribution of stars and dust plays a crucial role in determining the attenuation. There is a wide range of attenuations (1-2 mag) that can be found for a given observed (i.e., projected) dust mass surface density. The minimum values of attenuation are always found in Sc galaxies and later, while for the same amount of dust earlier spirals (especially Sb-Sbc ones) exhibit larger extinctions. In late-type spirals, dust and stars are arranged in a clumpy geometry: while some heating sources can be heavily attenuated, many UV photons can leak through lines of sight clean of dust, thus decreasing the overall attenuation. This does not happen so easily in the disks of Sbc spirals and earlier, where the same dust surface density yields larger attenuations.

8. By merging the dust mass profiles with $\mathrm{H}$ I data from the THINGS survey and CO data from the literature, we have studied the radial variation of the dust-to-gas ratio, which is seen to decrease by an order of magnitude from the center to the edge of the optical disk of each galaxy. Typical values range from $0.1-0.01$ in the central regions to $0.01-0.001$ in the outer ones, with early-type spirals having larger amounts of dust than late-type ones with a similar gas content. The dust-to-gas ratio is clearly correlated with the gas-phase oxygen abundance, being larger in the central, metal-rich zones of the disks than in the outer ones. To first order, for $12+\log (\mathrm{O} / \mathrm{H}>8.9)$, the relation can be described with a simple scaling law: $M_{\text {dust }} / M_{\text {gas }} \propto(\mathrm{O} / \mathrm{H})$, which would imply that most condensable elements tend to form dust in a roughly similar way in spiral galaxies of different morphological types. However, at lower metallicities, the dust-to-gas ratios are systematically below this simple relation. This could imply that large amounts of gas that has not yet undergone star formation activity reside in the outer regions of spiral disks, although the presence of dust cooler than $15 \mathrm{~K}$ could increase our dust-to-gas ratios.

J.C.M.M. acknowledges the receipt of a Formación del Profesorado Universitario fellowship from the Spanish Ministerio de Educación y Ciencia. J.C.M.M., A.G.d.P., J.Z., P.G.P., and J.G. are partially financed by the Spanish Programa Nacional de Astronomía y Astrofísica under grant AYA2006-02358. A.G.d.P. is also financed by the MAGPOP EU Marie Curie Research Training Network. We thank the anonymous referee for valuable comments that have improved the paper. We have made use of the NASA/IPAC Extragalactic Database (NED), which is operated by the Jet Propulsion Laboratory, California Institute of Technology (Caltech) under contract with NASA. GALEX is a NASA Small Explorer, launched in 2003 April. We gratefully acknowledge NASA's support for construction, operation, and science analysis for the GALEX mission, developed in cooperation with the Centre National d'Études Spatiales of France and the Korean Ministry of Science and Technology. This work is part of SINGS, the Spitzer Infrared Nearby Galaxies Survey. The Spitzer Space Telescope is operated by the Jet Propulsion Laboratory, Caltech, under NASA contract 1403. This work also makes use of data from THINGS, The H I Nearby Galaxies Survey. We thank A. Leroy and F. Walter for kindly providing the $\mathrm{H}$ I radial profiles. We also thank L. Cortese for providing his fit for Figure 5.

Facilities: GALEX, Spitzer, VLA

\section{APPENDIX A}

\section{EMPIRICAL ESTIMATIONS OF THE MODEL PARAMETERS}

Fitting the IR SEDs of star-forming galaxies with detailed models for the dust emission constitutes the proper way to analyze the physical properties of the interstellar dust. However, it is also desirable to have empirical calibrations from which one can roughly estimate these properties directly from observed quantities. Here we provide some basic equations for that purpose. In Table 5, we provide the residual rms of each one of these recipes.

\section{A.1. Estimating $\gamma$}

Draine \& Li (2007) already provide a useful fit to estimate $f\left(L_{d} ; U>10^{2}\right)$, that is, the fraction of the total dust luminosity contributed by high-intensity regions. They define the following quantity:

$$
P_{24}-0.14 P_{8.0} \equiv \frac{\left\langle v F_{v}^{\mathrm{ns}}\right\rangle_{24}-0.14\left\langle\nu F_{v}^{\mathrm{ns}}\right\rangle_{8.0}}{\left\langle v F_{v}\right\rangle_{70}+\left\langle\nu F_{v}\right\rangle_{160}},
$$

where the non-stellar flux densities $F_{v}^{\text {ns }}$ can be estimated using the stellar emission corrections given in Section 4.2. The numerator is the $24 \mu \mathrm{m}$ flux coming from large grains heated by very intense radiation fields. The factor $0.14\left\langle v F_{v}^{\mathrm{ns}}\right\rangle_{8.0}$ accounts 
Table 5

Accuracy of the Recipes

\begin{tabular}{|c|c|c|c|c|c|c|}
\hline \multirow{3}{*}{ Parameter $^{1}$} & & & \multicolumn{4}{|c|}{ Comparison with D07 } \\
\hline & \multicolumn{2}{|c|}{ Empirical Fit ${ }^{2}$} & \multicolumn{2}{|c|}{ No SCUBA $^{3}$} & \multicolumn{2}{|c|}{ SCUBA $^{4}$} \\
\hline & Offset & $\mathrm{rms}$ & Offset & $\mathrm{rms}$ & Offset & $\mathrm{rms}$ \\
\hline$q_{\mathrm{PAH}}$ & $0 \%$ & $\pm 11 \%$ & $+2.7 \%$ & $\pm 30 \%$ & $-1.1 \%$ & $\pm 14 \%$ \\
\hline$\gamma$ & $0 \%$ & $\pm 25 \%$ & $-5.7 \%$ & $\pm 23 \%$ & $-6.4 \%$ & $\pm 27 \%$ \\
\hline$\langle U\rangle$ & $0 \%$ & $\pm 9.2 \%$ & $-2.9 \%$ & $\pm 22 \%$ & $-22 \%$ & $\pm 35 \%$ \\
\hline$M_{\text {dust }}$ & $+5 \%$ & $\pm 9.6 \%$ & $+11 \%$ & $\pm 22 \%$ & $+30 \%$ & $\pm 39 \%$ \\
\hline $0.95 \times M_{\text {dust }}$ & $0 \%$ & $\pm 9.6 \%$ & $+5.8 \%$ & $\pm 22 \%$ & $+25 \%$ & $\pm 39 \%$ \\
\hline
\end{tabular}

Notes. Accuracy of the empirical recipes for each model parameter (Appendix A) and comparison with the results of Draine et al. (2007) (Appendix B). (1) Each one of the dust model parameters. (2) Offset and rms of the empirical fits. By definition, the offset is zero for all parameters, since the corresponding estimators are derived from fits applied to our data. The only exception is the dust mass, for which the empirical recipe is directly obtained by combining the estimators for $\langle U\rangle$ and $L_{\text {dust }}$. This leads to dust masses $5 \%$ larger than the ones yielded by the model (see Appendix A.4). If desired, this can be accounted for by multiplying by 0.95 (last row). (3) Comparison between the values published by D07, obtained by fitting the global SEDs including data from 2MASS, IRAC, MIPS, and IRAS, and the ones we estimate by applying our recipes to their IRAC and MIPS data alone. The first number indicates the relative offset (our value/D07 value) and the second one the rms. (4) Same as (3), but for the galaxies for which submillimeter data from SCUBA were added to the data set used in D07.

for the emission at $24 \mu \mathrm{m}$ contributed by stochastically heated PAHs, since this emission is not necessarily linked to highintensity regions. DL07 showed that $f\left(L_{d} ; U>10^{2}\right)$ can be estimated using the following fitting function:

$$
f\left(L_{d} ; U>10^{2}\right)(\%)=105\left(P_{24}-0.14 P_{8.0}-0.035\right)^{0.75} \text {. }
$$

While DL07 showed that this function is certainly a good proxy for the fraction of the dust luminosity coming from highintensity regions, Figure 17(a) shows that this is also the case for our radial profiles. The dispersion in the lower region is due to differences in $U_{\min }$ and $q_{\mathrm{PAH}}$.

The corresponding mass fraction enclosed in these regions is also tightly correlated with $P_{24}-0.14 P_{8.0}$, as can be seen in panel (b). Following DL07, we performed a similar fit to obtain an estimator for $\gamma$ :

$$
\gamma(\%)=46.2\left(P_{24}-0.14 P_{8.0}-0.023\right)^{1.28} .
$$

\section{A.2. Estimating $q_{\mathrm{PAH}}$}

The emission at $8.0 \mu \mathrm{m}$ depends on both the PAH abundance and the amount of dust exposed to high-intensity starlight. Therefore, one would expect that $q_{\mathrm{PAH}}$ could be obtained from $P_{8.0}$ and $P_{24}-0.14 P_{8.0}$, the latter accounting for differences in $\gamma$, as seen above. This is confirmed in Figure 18(a), where we can see that the PAH abundance correlates well with $P_{8.0}$, with a second-order dependence on $P_{24}-0.14 P_{8.0}$. As expected, at fixed $q_{\mathrm{PAH}}$ the emission at $8.0 \mu \mathrm{m}$-relative to the emission at longer wavelengths -increases with $\gamma$.

A close inspection of data points distribution reveals that a certain linear combination of $P_{8.0}$ and $P_{24}$ would allow estimating $q_{\mathrm{PAH}}$ with a single fit. We computed this linear combination by means of a Principal Component Analysis. The results are shown in panel (b), where we can see that the PAH abundance can be readily estimated using the following fitting function:

$$
q_{\mathrm{PAH}}(\%)=13.03\left(P_{8.0}-0.38 P_{24}+0.022\right)^{0.77} .
$$
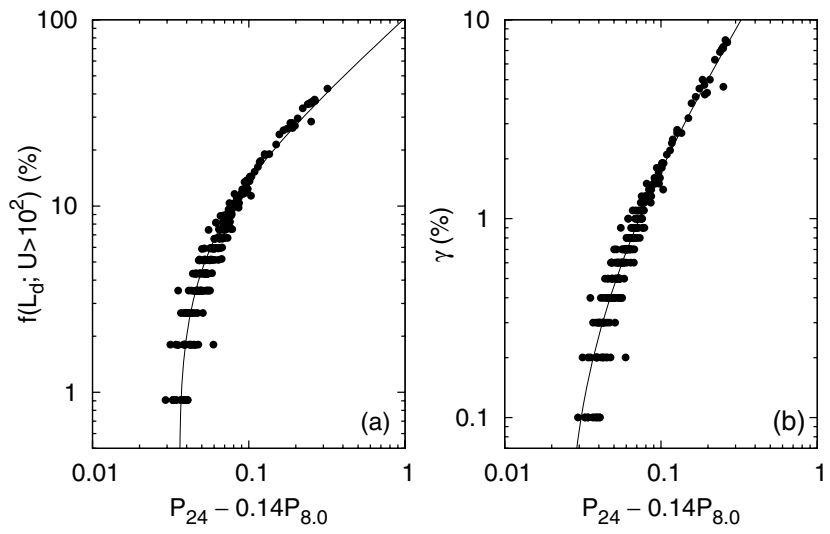

Figure 17. (a) Fraction of the total dust luminosity contributed by grains in regions where $U>10^{2}$ as a function of the observable $P_{24}-0.14 P_{8.0}$ (see the text). The solid line is the theoretical fitting function provided by Draine \& Li (2007). (b) Relation between the fraction of the dust mass enclosed in high-intensity regions and $P_{24}-0.14 P_{8.0}$. The solid line is the best-fit function of our radial profiles.

Since the largest value of the PAH abundance derived from the models is $q_{\mathrm{PAH}}=4.6 \%$, there are many data points saturating that region of the plot-their actual PAH abundances being possibly larger - so we excluded those points in this analysis. For the same reason, points with $q_{\mathrm{PAH}}=0.4 \%$ were also excluded, since this is the lower limit of the dust models. Nevertheless, our fit should still allow us to recover PAH abundances a few percent larger than the current upper limit, since there is no reason a priori for this trend to break just above that value.

\section{A.3. Estimating $\langle U\rangle$}

The scale factor of the dust-weighted mean starlight intensity, $\langle U\rangle$, depends strongly on the shape of the FIR SED. In Figure 19, we show that this parameter is linearly correlatedin logarithmic scale-with $R_{70} \equiv\left\langle v F_{v}\right\rangle_{70} /\left\langle\nu F_{v}\right\rangle_{160}$, which is sensitive to the temperature of the largest grains dominating the FIR emission. A linear fit yields the following empirical function:

$$
\log \langle U\rangle=0.468+1.801 \log R_{70} .
$$

The starlight intensity of the diffuse component, $U_{\min }$, is not so tightly correlated with $R_{70}$, since the effect of $\gamma$ must be taken into account. Once $\langle U\rangle$ and $\gamma$ have been estimated using the formulae above, $U_{\min }$ can be computed from Equation (33) in DL07.

Note that, as explained in Section 4, the smallest value of $U_{\min }$ considered in the model-fitting procedure is 0.7 , as DL07 suggest to proceed when lacking submillimeter data. As a consequence, the resulting fits also have $\langle U\rangle \geqslant 0.7$. For this reason, some data points are clustered in the lower region of Figure 19, and have not been taken into account when performing the fit.

\section{A.4. Estimating the Dust Mass}

We can estimate the dust mass from the mean starlight intensity $\langle U\rangle$ and the dust luminosity, since

$$
L_{\text {dust }}=P_{0}\langle U\rangle M_{\text {dust }},
$$

where $P_{0}$ is the power per unit of mass radiated by the dust, when exposed to a radiation field equal to the local MW one (i.e., with $U=1$ ). According to the DL07 models, differences in the PAH 


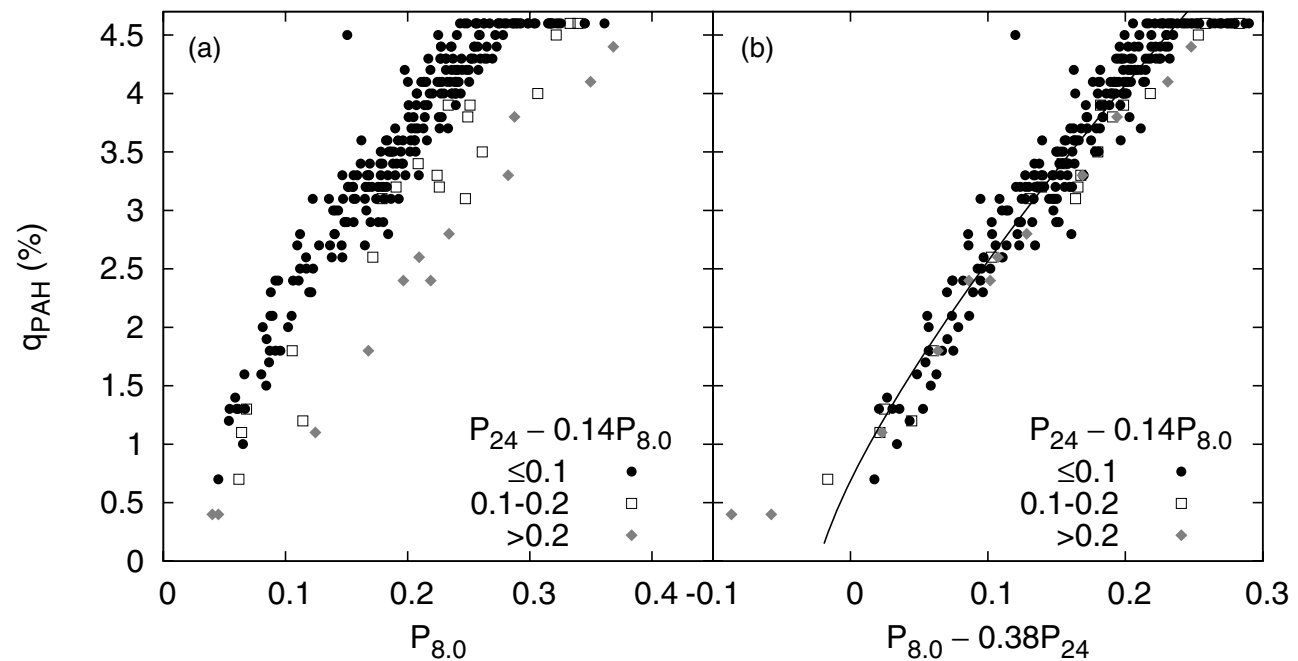

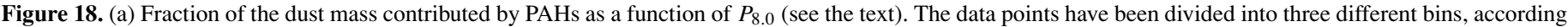

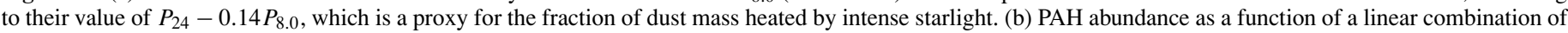

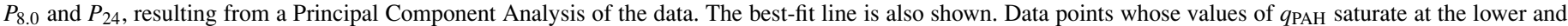
upper limits of the dust model $(0.4 \%$ and $4.6 \%$, respectively) were not taken into account when performing the fit.

abundance can introduce variations of $\sim 2 \%$ in this parameter. Nevertheless, a constant average value of $P_{0} \approx 137 L_{\odot} / M_{\odot}$ can be safely adopted.

The dust luminosity can be estimated directly from the photometric data using the weighted sum proposed by DL07. By substituting that sum and the empirical fit for $\langle U\rangle$ (Equation (A5)) into Equation (A6), we can derive the dust mass from the IRAC and MIPS flux densities and the distance to the source:

$$
\begin{aligned}
\frac{M_{\text {dust }}}{M_{\odot}}= & \frac{4 \pi}{1.616 \times 10^{-13}}\left(\frac{D}{\mathrm{Mpc}}\right)^{2}\left(\frac{\left\langle v F_{v}\right\rangle_{70}}{\left\langle v F_{v}\right\rangle_{160}}\right)^{-1.801} \\
& \times \frac{0.95\left\langle v F_{v}^{\mathrm{ns}}\right\rangle_{8.0}+1.150\left\langle v F_{v}^{\mathrm{ns}}\right\rangle_{24}+\left\langle v F_{v}\right\rangle_{70}+\left\langle v F_{v}\right\rangle_{160}}{\operatorname{erg~s}^{-1} \mathrm{~cm}^{-2}} .
\end{aligned}
$$

As in previous sections, the non-stellar flux densities $F_{v}^{\text {ns }}$ can be obtained from the observed ones by extrapolating the stellar continuum from the observed $3.6 \mu$ m flux density (see Section 4.2). To check the validity of this estimator, we have applied it to our radial profiles. In Figure 20(a), we compare the dust mass surface densities obtained from the model-fitting procedure with the ones estimated through Equation (A7). The agreement is excellent, with a scatter of only $9 \%$. The most deviant points are those with the smallest value of the diffuse starlight intensity considered throughout this work (see Appendix A.3). Note that there is a small offset between the dust masses derived from Equation (A7) and the actual ones obtained from the model fitting, due to the fact that the $L_{\text {dust }}$ estimator slightly overestimates the actual $L_{\text {dust }}$ from the model for the particular range of values of $\langle U\rangle$ and $q_{\mathrm{PAH}}$ in our galaxies. Although Equation (A7) is intended to be more general, if one wishes to account for this fact, the dust masses derived from Equation (A7) should be multiplied by 0.95 (see Table 5).

In the absence of IRAC data, we can still compute the dust mass simply by substituting the $L_{\mathrm{TIR}}$ estimator of DL07 (i.e., the numerator of the last term in Equation (A7)) with the MIPSonly calibration of Dale \& Helou (2002). We can also skip the stellar emission correction for the $24 \mu \mathrm{m}$ band, since it is almost

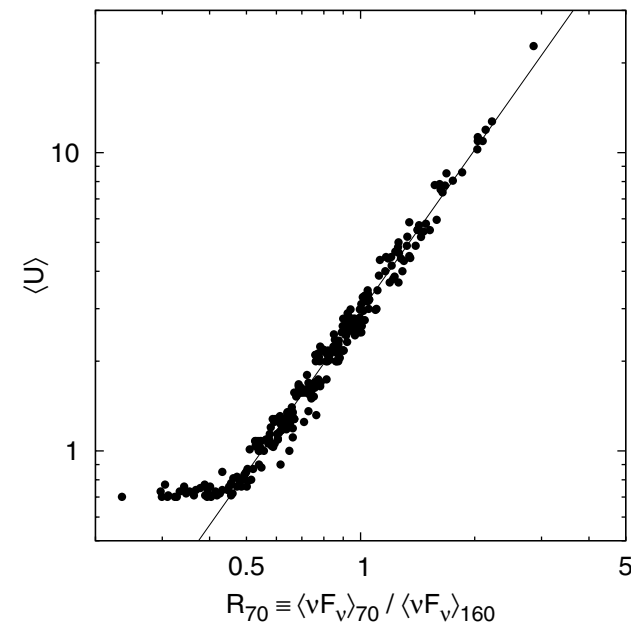

Figure 19. Scale factor of the dust-weighted mean starlight intensity-in units of the local MW radiation field-against the ratio of the luminosities at 70 and $160 \mu \mathrm{m}$. The best-fitting line is also shown. Data points with $\langle U\rangle \approx 0.7$ have been excluded from the fit (see the text for details).

negligible. Hence, we have

$$
\begin{aligned}
\frac{M_{\text {dust }}}{M_{\odot}}= & \frac{4 \pi}{1.616 \times 10^{-13}}\left(\frac{D}{\mathrm{Mpc}}\right)^{2}\left(\frac{\left\langle v F_{v}\right\rangle_{70}}{\left\langle v F_{v}\right\rangle_{160}}\right)^{-1.801} \\
& \times \frac{1.559\left\langle v F_{v}\right\rangle_{24}+0.7686\left\langle v F_{v}\right\rangle_{70}+1.347\left\langle v F_{v}\right\rangle_{160}}{\operatorname{erg~s}^{-1} \mathrm{~cm}^{-2}} .
\end{aligned}
$$

In Figure 20(b), we see that this expression also constitutes a good metric tracer for the dust mass. Note, however, that the dust masses derived in this paper have been obtained in the absence of spatially resolved submillimeter data, and therefore the amount of dust colder than $15 \mathrm{~K}$ might not be strongly constrained. When analyzing the integrated properties of the SINGS galaxies, D07 showed that omitting submillimeter data can lead to an uncertainty of $\sim 50 \%$ in the dust mass, and this should be taken into account when using the previous equations to directly compute the dust masses. 


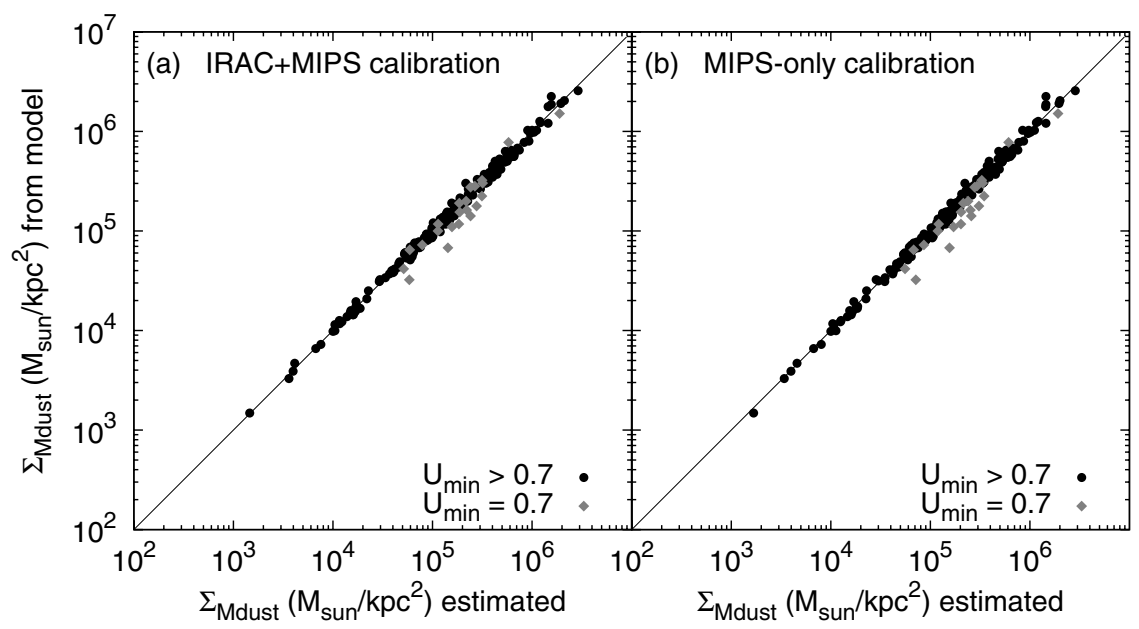

Figure 20. Comparison of the dust mass surface densities obtained from the model-fitting technique and from the estimator given in Appendix A.4, either with the IRAC+MIPS calibration (Equation (A7), panel a) or with the MIPS-only one (Equation (A8), panel b). Note that since we are dealing with surface densities, the results do not depend on the distance to the sources. Regions for which the fitting procedure "saturates" at the lowest value of the diffuse starlight intensity $U_{\text {min }}$ have been marked.

\section{APPENDIX B \\ POSSIBLE SYSTEMATIC EFFECTS IN THE DUST MODELS}

When using the DL07 dust models to fit our radial IR SEDs, we have relied only on IRAC and MIPS data. Hence, it is worth analyzing the possible systematics effects that may arise from using a limited number of bands to constrain the models. D07 made use of a larger set of multi-wavelength data to study the global properties of the SINGS galaxies. Besides IRAC and MIPS observations, they included in their fit data from Two Micron All Sky Survey (2MASS), IRAS, SCUBA (for 17 galaxies), and the IRS "blue peak-up" detector array onboard Spitzer (for nine galaxies). While useful for global analysis, the use of these additional data sets in a spatially resolved analysis poses some problems regarding resolution, depth, and heterogeneous data quality.

To assess the validity of our methodology, we have compared our values of the model parameters with the ones derived by D07 for each galaxy as a whole. In principle, given a radial profile for each parameter in a certain galaxy, it is not straightforward to reduce "a posteriori" the spatially resolved information into a single data point representative of the whole galaxy. A more reliable and robust procedure consists of applying the empirical formulae presented in Appendix A to the IRAC and MIPS integrated photometry used in D07. These recipes are affected by the same systematic effects, if any, as the values directly yielded by our model-fitting procedure, since they were explicitly calibrated using those data. Should there be any strong biases in our values, they should arise when comparing our estimates with the values computed by D07.

Table 5 quotes the offsets and scatter between our values and those published in D07. Note that part of the scatter is due to the intrinsic rms of each recipe. In Figure 21, we compare the D07 values with the ones estimated using our recipes. Panel (a) shows that our PAH abundances might be overestimated at very low abundances, and underestimated at the largest ones. The overall relative scatter is $\sim 30 \%$.

In panel (b), we compare the values of the fraction of dust mass heated by very intense starlight. The correlation is
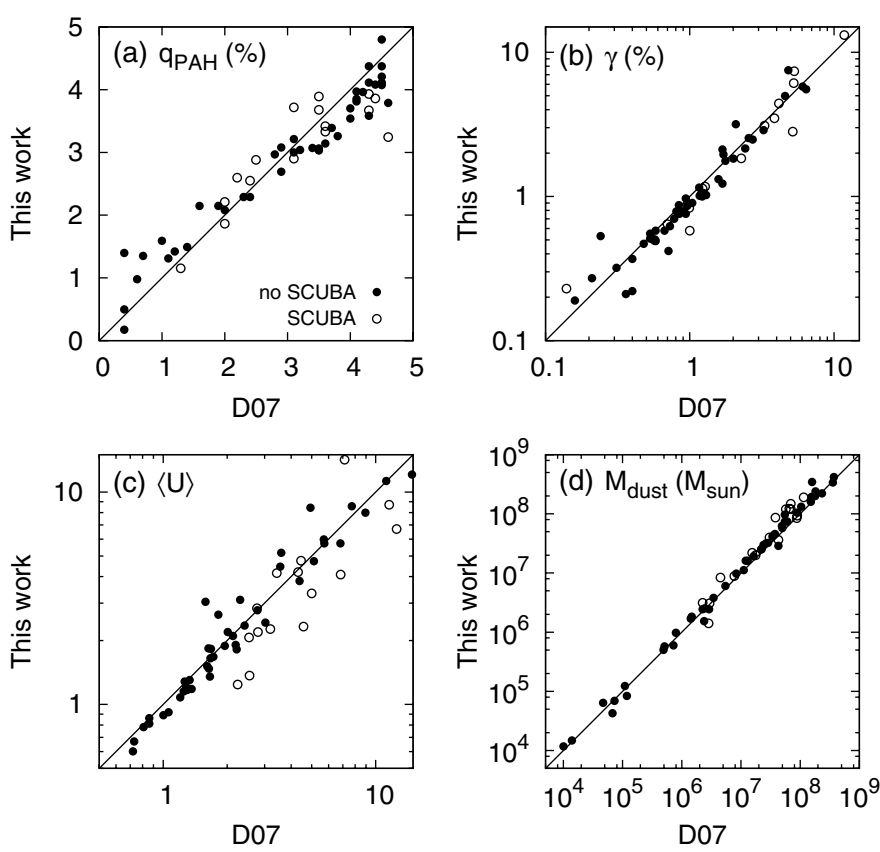

Figure 21. Comparison of the values of the dust model parameters derived by D07 with the ones estimated using our recipes. The former were obtained by using global photometry from 2MASS, IRAS, and Spitzer to constrain the dust models. Our estimates were computed by applying the empirical formulae described in Appendix A to the integrated IRAC and MIPS data alone. Open circles correspond to galaxies for which submillimeter data from SCUBA were used by D07. The mean offsets and scatter are quoted in Table 5. (a) Abundance of PAHs. NGC 1291, NGC 1316, NGC 4125, and NGC 4594 have been excluded, since their IRAC $8.0 \mu \mathrm{m}$ flux densities are highly contaminated by stellar emission, leading to very uncertain values of $q_{\mathrm{PAH}}$. (b) Fraction of the dust mass heated by intense starlight. (c) Dust-weighted mean starlight intensity, in units of the local MW radiation field. (d) Total dust mass.

excellent, although our values are systematically $6 \%$ lower, with an rms of $\sim 25 \%$.

The average intensity of the radiation field heating the dust that we get from our SEDs is just 3\% lower than the one from D07, with a scatter of $22 \%$, as can be seen in panel (c). The most deviant points contributing to the dispersion 
are those with available SCUBA data, for which we get lower dust temperatures.

As a result, our dust masses appear to be just slightly overestimated in comparison with those obtained by D07 (see panel d). For galaxies lacking SCUBA data, our masses are only $6 \%$ larger, with an rms of $\sim 22 \%$. In galaxies with available submillimeter data, the offset increases up to $25 \%$, with a scatter of $40 \%$.

In brief, the correlations shown in Figure 21 are good enough to assert that the results presented in this paper do not suffer from any strong bias due to our restricted wavelength range that could compromise the validity of our conclusions.

\section{REFERENCES}

Bajaja, E., Wielebinski, R., Reuter, H. P., Harnett, J. I., \& Hummel, E. 1995, A\&A, 114, 147

Bell, E. F. 2002, ApJ, 577, 150

Bell, E. F., \& de Jong, R. S. 2000, MNRAS, 312, 497

Bendo, G. J., et al. 2006, ApJ, 652, 283

Bendo, G. J., et al. 2007, MNRAS, 380, 1313

Bendo, G. J., et al. 2008, MNRAS, 389, 629

Boissier, S., Boselli, A., Buat, V., Donas, J., \& Milliard, B. 2004, A\&A, 424, 465

Boissier, S., \& Prantzos, N. 1999, MNRAS, 307, 857

Boissier, S., et al. 2005, ApJ, 619, 83

Boissier, S., et al. 2007, ApJS, 173, 524

Boselli, A., Boissier, S., Cortese, L., Gil de Paz, A., Seibert, M., Madore, B. F., Buat, V., \& Martin, D. C. 2006, ApJ, 651, 811

Boselli, A., \& Gavazzi, G. 2006, PASP, 118, 517

Boselli, A., Lequeux, J., \& Gavazzi, G. 2002, A\&A, 384, 33

Boselli, A., et al. 1998, A\&A, 335, 53

Boselli, A., et al. 2005, ApJ, 629, 29

Brinchmann, J., Charlot, S., White, S. D. M., Tremonti, C., Kauffmann, G., Heckman, T., \& Brinkmann, J. 2004, MNRAS, 351, 1151

Bruzual, G., \& Charlot, S. 2003, MNRAS, 344, 1000

Buat, V., \& Xu, C. 1996, A\&A, 306, 61

Buat, V., et al. 2005, ApJ, 619, 51

Burgarella, D., Buat, V., \& Iglesias-Páramo, J. 2005, MNRAS, 360, 1413

Burstein, D., Bertola, F., Buson, L. M., Faber, S. M., \& Lauer, T. R. 1988, ApJ, 328,440

Calzetti, D. 2001, PASP, 113, 1449

Calzetti, D., Kinney, A. L., \& Storchi-Bergmann, T. 1994, ApJ, 429, 582

Calzetti, D., et al. 2005, ApJ, 633, 871

Cardiel, N. 2009, MNRAS, 396, 680

Cortese, L., Boselli, A., Franzetti, P., Decarli, R., Gavazzi, G., Boissier, S., \& Buat, V. 2008, MNRAS, 386, 1157

Cortese, L., et al. 2006, ApJ, 637, 242

da Cunha, E., Charlot, S., \& Elbaz, D. 2008, MNRAS, 388, 1595

Dale, D. A., \& Helou, G. 2002, ApJ, 576, 159

Dale, D. A., Helou, G., Contursi, A., Silbermann, N. A., \& Kolhatkar, S. 2001, ApJ, 549, 215

Dale, D. A., et al. 2007, ApJ, 655, 863

de Jong, R. S. 1996, A\&A, 313, 377

de Vaucouleurs, G., de Vaucouleurs, A., Corwin, H. G., Buta, R. J., Paturel, G., \& Fouqué, P. 1991, Third Reference Catalogue of Bright Galaxies (RC3) (Berlin: Springer)

Draine, B. T. 2009, in ASP Conf. Ser., Cosmic Dust-Near and Far, ed. T. Henning, E. Grün, \& J. Steinacker (San Francisco, CA: ASP), in press (arXiv:0903.1658)

Draine, B. T., \& Li, A. 2007, ApJ, 657, 810 (DL07)

Draine, B. T., et al. 2007, ApJ, 663, 866 (D07)

Dwek, E., Galliano, F., \& Jones, A. 2009, in ASP Conf. Ser., Cosmic DustNear and Far, ed. T. Henning, E. Grün, \& J. Steinacker (San Francisco, CA: ASP), in press (arXiv:0903.0006)

Edmunds, M. G. 2001, MNRAS, 328, 223

Engelbracht, C. W., Gordon, K. D., Rieke, G. H., Werner, M. W., Dale, D. A., \& Latter, W. B. 2005, ApJ, 628, 29

Engelbracht, C. W., Rieke, G. H., Gordon, K. D., Smith, J. D. T., Werner, M. W., Moustakas, J., Willmer, C. N. A., \& Vanzi, L. 2008, ApJ, 678, 804

Engelbracht, C. W., et al. 2007, PASP, 119, 994

Fazio, G. G., et al. 2004, ApJS, 154, 10

Galliano, F., Dwek, E., \& Chanial, P. 2008, ApJ, 672, 214
García-Lario, P. 2006, in IAU Symp. 234, Planetary Nebulae in Our Galaxy and Beyond, ed. M. J. Barlow \& R. H. Méndez (Cambridge: Cambridge Univ. Press), 63

Gavazzi, G., Bonfanti, C., Sanvito, G., Boselli, A., \& Scodeggio, M. 2002, ApJ, 576,135

Gil de Paz, A., \& Madore, B. F. 2005, ApJS, 156, 345

Gil de Paz, A., et al. 2007, ApJS, 173, 185

Gordon, K. D., Clayton, G. C., Witt, A. N., \& Misselt, K. A. 2000, ApJ, 533, 236

Gordon, K. D., Engelbracht, C. W., Rieke, G. H., Misselt, K. A., Smith, J. D. T., \& Kennicutt, R. C., Jr. 2008, ApJ, 682, 336

Gordon, K. D., et al. 2007, PASP, 119, 1019

Heckman, T. M., Robert, C., Leitherer, C., Garnett, D. R., \& van der Rydt, F. 1998, ApJ, 503, 646

Heckman, T. M., et al. 1995, ApJ, 452, 549

Helou, G., et al. 2004, ApJS, 154, 253

Hirashita, H., Tajiri, Y. Y., \& Kamaya, H. 2002, A\&A, 388, 439

Hogg, D. W., Tremonti, C. A., Blanton, M. R., Finkbeiner, D. P., Padmanabhan, N., Quintero, A. D., Schlegel, D. J., \& Wherry, N. 2005, ApJ, 624, 162

Holwerda, B. W., Gonzalez, R. A., Allen, R. J., \& van der Kruit, P. C. 2005, AJ, 129,1396

Hunt, L., Bianchi, S., \& Maiolino, R. 2005, A\&A, 434, 849

Issa, M. R., MacLaren, I., \& Wolfendale, A. W. 1990, A\&A, 236, 237

James, A., Dunne, L., Eales, S., \& Edmunds, M. G. 2002, MNRAS, 335, 753

Kenney, J., \& Young, J. 1988, ApJS, 66, 261

Kennicutt, R. C., Jr., et al. 2003, PASP, 115, 928

Kobulnicky, H. A., \& Kewley, L. J. 2004, ApJ, 617, 240

Kong, X., Charlot, S., Brinchmann, J., \& Fall, S. M. 2004, MNRAS, 349, 769

Leitherer, C., et al. 1999, ApJS, 123, 3

Leroy, A. K., Walter, F., Brinks, E., Bigiel, F., de Blok, W. J. G., Madore, B. F., \& Thornley, M. D. 2008, AJ, 136, 2782

Li, A., \& Draine, B. T. 2001, ApJ, 554, 778

Lisenfeld, U., \& Ferrara, A. 1998, ApJ, 496, 145

MacArthur, L. A., Courteau, S., Bell, E., \& Holtzman, J. A. 2004, ApJS, 152, 175

Madden, S. C. 2000, New Astron. Rev., 44, 249

Madden, S. C., Galliano, F., Jones, A. P., \& Sauvage, M. 2006, A\&A, 446, 877 Martin, D., et al. 2005, ApJ, 619, L1

Mathis, J. S., Mezger, P. G., \& Panagia, N. 1983, A\&A, 128, 212

Meurer, G. R., Heckman, T. M., \& Calzetti, D. 1999, ApJ, 521, 64

Meurer, G. R., Heckman, T. M., Leitherer, C., Kinney, A., Robert, C., \& Garnett, D. R. 1995, AJ, 110, 2665

Muñoz-Mateos, J. C., et al. 2007, ApJ, 658, 1006

Muñoz-Mateos, J. C., et al. 2009, ApJ, submitted

O'Connell, R. W. 1999, ARA\&A, 37, 603

Paglione, T. A. D., et al. 2001, ApJS, 135, 183

Panuzzo, P., Granato, G. L., Buat, V., Inoue, A. K., Silva, L., Iglesias-Páramo, J., \& Bressan, A. 2007, MNRAS, 375, 640

Pérez-González, P. G., Gil de Paz, A., Zamorano, J., Gallego, J., Alonso-Herrero, A., \& Aragón-Salamanca, A. 2003, MNRAS, 338, 525

Pérez-González, P. G., et al. 2006, ApJ, 648, 987

Pilyugin, L. S., \& Thuan, T. X. 2005, ApJ, 631, 231

Popescu, C. C., Misiriotis, A., Kylafis, N. D., Tuffs, R. J., \& Fischera, J. 2000, A\&A, 362, 138

Popescu, C. C., et al. 2005, ApJ, 619, 75

Prescott, M. K. M., et al. 2007, ApJ, 668, 182

Press, W. H., Teukolsky, S. A., Vetterling, W. T., \& Flannery, B. P. 1992, Numerical Recipes in C: The Art of Scientific Computing (Cambridge: Univ. Press)

Reach, W. T., et al. 2005, PASP, 117, 978

Regan, M. W., Thornley, M. D., Helfer, T. T., Sheth, K., Wong, T., Vogel, S. N., Blitz, L., \& Bock, D. C. J. 2001, ApJ, 561, 218

Rieke, G. H., et al. 2004, ApJS, 154, 25

Roussel, H., Gil de Paz, A., Seibert, M., Helou, G., Madore, B. F., \& Martin, C. 2005, ApJ, 632, 227

Rubin, R. H., et al. 2008, MNRAS, 387, 45

Sage, L. J. 1993, A\&A, 272, 123

Scalo, J. M. 1986, Fundam. Cosm. Phys., 11, 1

Schlegel, D. J., Finkbeiner, D. P., \& Davis, M. 1998, ApJ, 500, 525

Seibert, M., et al. 2005, ApJ, 619, 55

Smith, J. D. T., et al. 2007, ApJ, 656, 770

Stansberry, J. A., et al. 2007, PASP, 119, 1038

Sturm, E., Lutz, D., Tran, D., Feuchtgruber, H., Genzel, R., Kunze, D. Moorwood, A. F. M., \& Thornley, M. D. 2000, A\&A, 358, 481

Taylor, V. A., Jansen, R. A., Windhorst, R. A., Odewahn, S. C., \& Hibbard, J. E. 2005, ApJ, 630, 784 
Thilker, D. A., et al. 2007, ApJS, 173, 572

van den Bergh, S. 1976, ApJ, 206, 883

Walter, F., Brinks, E., de Blok, W. J. G., Bigiel, F., Kennicutt, R. C., Jr., Thornley, M. D., \& Leroy, A. K. 2008, AJ, 136, 2563

Walter, F., et al. 2007, ApJ, 661, 102

Weingartner, J. C., \& Draine, B. T. 2001, ApJ, 548, 296

Werner, M. W., et al. 2004, ApJS, 154, 1
Witt, A. N., \& Gordon, K. D. 2000, ApJ, 528, 799

Wu, Y., Charmandaris, V., Hao, L., Brandl, B. R., Bernard-Salas, J., Spoon, H. W. W., \& Houck, J. R. 2006, ApJ, 639, 157

Xilouris, E. M., Byun, Y. L., Kylafis, N. D., Paleologou, E. V., \& Papamastorakis, J. 1999, A\&A, 344, 868

Young, J. S., \& Scoville, N. 1982, ApJ, 260, 41

Young, J. S., et al. 1995, ApJS, 98, 219 